\title{
Concise Synthesis of Enantiopure $\alpha$-Trifluoromethyl alanines, Diamines and Amino Alcohols via the Strecker-type Reaction
}

\author{
Florent Huguenot ${ }^{\dagger}$ and Thierry Brigaud ${ }^{*} \ddagger$
}

Laboratoire "Réactions Sélectives et Applications", UMR CNRS 6519, Université de ReimsChampagne-Ardenne, Faculté des Sciences, BP 1039, 51687 Reims Cedex 2, France, and Laboratoire "Synthèse Organique Sélective et Chimie Organométallique " (SOSCO), UMR CNRS 8123, Université de Cergy-Pontoise,5, Mail Gay-Lussac - Neuville sur Oise - 95031 Cergy-Pontoise cedex, France.

\section{thierry.brigaud@u-cergy.fr}

\section{Table of Contents}

General information S2

Preparation and characterization data of imines 1a-g $\quad$ S2

Preparation and characterization data of amino nitriles $\mathbf{2} \mathbf{a}-\mathbf{g}$ from imines $\mathbf{1 a - g} \quad$ S4

Preparation and characterization data of amino nitriles $\mathbf{4 a}, \mathbf{b}$ from silylhemiacetals $\mathbf{3 a}, \mathbf{b} \quad$ S8

Procedure and characterization data of oxazolidines $\mathbf{5 a - c}$ and $\mathbf{7 a - e} \quad \mathrm{S} 10$

Preparation and characterization data of amino nitriles $\mathbf{6 a}-\mathbf{c}, \mathbf{8 a}, \mathbf{b}, \mathbf{d}$

$\begin{array}{lr}\text { from oxazolidines } \mathbf{5 a}-\mathbf{c}, \mathbf{7 a}, \mathbf{b}, \mathbf{d} & \text { S14 }\end{array}$

$\begin{array}{ll}\text { Preparation and characterization data of }(R) \text { - and }(S) \text {-Trifluoromethyl alanine } & \text { S18 }\end{array}$

Preparation and characterization data of compounds 13d,e 14e, 15a,e S20

Copies of ${ }^{1} \mathrm{H}$ and ${ }^{13} \mathrm{C}$ NMR spectra of compounds 1f, 1g, 2b, 2c, 2d, 2g, 3a, 4a, 4b, 5b, 5c,

7b, 6a, 6c, 13d, 15a and 15e 


\section{General information}

Unless otherwise mentioned, all the reagents were purchased from commercial source. All glassware was dried in an oven at $150{ }^{\circ} \mathrm{C}$ prior to use. Ether and THF were distilled under nitrogen from sodium/benzophenone prior to use. $\mathrm{CH}_{2} \mathrm{Cl}_{2}$ was distilled under nitrogen from $\mathrm{CaH}_{2}$ prior to use. Chemical shifts of ${ }^{1} \mathrm{H}$ NMR $(250,13 \mathrm{MHz})$ were expressed in parts per million downfield from tetramethylsilane $(\delta=0)$ in $\mathrm{CDCl}_{3}$. Chemical shifts of ${ }^{13} \mathrm{C} \mathrm{NMR}$ $(62,89 \mathrm{MHz})$ were expressed in parts per million downfield from $\mathrm{CDCl}_{3}$ as internal standard $(\delta=77.0)$. Chemical shifts of ${ }^{19} \mathrm{~F}$ NMR $(235,36 \mathrm{MHz})$ were expressed in parts per million downfield from $\mathrm{CFCl}_{3}$ as internal standard $(\delta=0)$. Coupling constants are reported in hertz. Column chromatography was performed on $0,040-0,063 \mathrm{~mm}$ silica gel, employing mixture of petroleum ether and ethyl acetate as eluent. Silica TLC plates were visualized under UV light, by a $10 \%$ solution of phosphomolybdic acid in ethanol followed by heating. Gas chromatography (FID detection) was performed with a polydimethylsiloxane column $(25 \mathrm{~m} \mathrm{X}$ $3,2 \mathrm{~mm} \mathrm{X} 0,52 \mu \mathrm{m}$ thickness of layer). Mass Spectrometry was carried out in the EI mode. High Resolution Mass Spectrometry was carried out by using electrospray method. Wavenumbers of infrared spectra (IR)are given in $\mathrm{cm}^{-1}$. Optical rotations are reported as their specific rotations at $25^{\circ} \mathrm{C}$ in $\mathrm{g} / 100 \mathrm{~mL}$. Melting points are uncorrected.

\section{Preparation of imines 1a-g.}

Imines $\mathbf{1 a},{ }^{1} \mathbf{1} \mathbf{b},{ }^{2} \mathbf{1} \mathbf{c}^{2}$ and $\mathbf{1} \mathbf{d}^{1}$ were prepared according to literature procedures.

(1) Legros, J.; Meyer, F.; Coliboeuf, M.; Crousse, B.; Bonnet-Delpon, D.; Bégué, J.-P. J. Org. Chem. 2003, 68, 6444-6446.

(2) Soloshonok, V. A.; Ono, T. J. Org. Chem. 1997; 62, 3030-3031. 


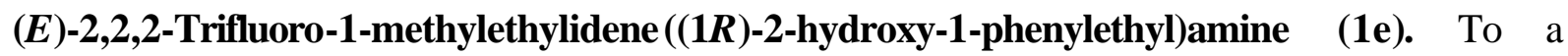
stirred solution of LDA (1.1 mmol, 1.1 equiv) in dry THF $(5 \mathrm{~mL})$ under argon at $-78^{\circ} \mathrm{C}$, was added a solution of oxazolidine $7 \mathbf{e}(230 \mathrm{mg}, 1.0 \mathrm{mmol})$ in THF (1 mL). After complete conversion of the substrate, the reaction mixture was quenched by addition of a saturated $\mathrm{NaHCO}_{3}$ solution $(5 \mathrm{~mL})$. The aqueous layer was extracted with diethyl ether $(3 \times 10 \mathrm{~mL})$ and the combined organic extracts were dried over $\mathrm{Na}_{2} \mathrm{SO}_{4}$, filtered, and concentrated under reduced pressure. The crude product (213 mg, 92\%) was used without further purification. Pale yellow oil; $[\alpha]^{20}{ }_{\mathrm{D}}-69.0\left(c\right.$ 1.4, $\mathrm{CHCl}_{3}$ ); IR (neat) 3364, 3032, 2934, 1688, 1454, 1126 $\mathrm{cm}^{-1} ;{ }^{1} \mathrm{H}$ NMR $\delta 2.30(\mathrm{bs}, 1 \mathrm{H}), 2.55(\mathrm{~s}, 3 \mathrm{H}), 3.70\left(\mathrm{dd}, J_{\mathrm{AB}}=10.1, J=8.7 \mathrm{~Hz}, 1 \mathrm{H}\right), 3.79(\mathrm{dd}$, $\left.J_{\mathrm{AB}}=10.1, J=4.4 \mathrm{~Hz}, 1 \mathrm{H}\right), 4.71(\mathrm{dd}, J=8.7,4.4 \mathrm{~Hz}, 1 \mathrm{H}), 7.10-7.40(\mathrm{~m}, 5 \mathrm{H}),{ }^{13} \mathrm{C} \mathrm{NMR}$ $\delta 13.6,66.6,67.8,119.7(\mathrm{q}, J=278.3 \mathrm{~Hz}), 127.2,127.8,128.7,138.7,158.5(\mathrm{q}, J=33.3 \mathrm{~Hz})$; ${ }^{19}$ F NMR $\delta-74.8(\mathrm{~s}) ;$ MS (EI $\left.70 \mathrm{eV}\right) \mathrm{m} / \mathrm{z} 332(\mathrm{M}++1), 214,200,103$ (100), 77.

\section{(E)-2,2,2-Trifluoro-1-methylethylidene ((1R)-2-tert-butyldimethylsilyloxy-1-phenylethyl)-}

amine (1f). To a solution of imine 1e $(230 \mathrm{~g}, 1.0 \mathrm{mmol})$, triethylamine $(0.28 \mathrm{~mL}, 2.0 \mathrm{mmol})$ and DMAP (10 mg, $0.1 \mathrm{mmol})$ in $\mathrm{CH}_{2} \mathrm{Cl}_{2}(5 \mathrm{~mL})$ under argon atmosphere, was added a solution of TBDMSCl $(0.16 \mathrm{~g}, 1.1 \mathrm{mmol})$ in $\mathrm{CH}_{2} \mathrm{Cl}_{2}(1 \mathrm{~mL})$ at room temperature. After was $24 \mathrm{~h}$ Stirring at room temperature, the reaction mixture was filtered on silica gel with diethyl ether. The resulting solution was concentrated under reduced pressure to afford quantitatively 1f as a yellow oil. $[\alpha]^{25}-17.3\left(c\right.$ 1.6, $\left.\mathrm{CHCl}_{3}\right)$; IR (neat) 3064, 2955, 2930, 1604, 1338, 1200, $1128 \mathrm{~cm}^{-1} ;{ }^{1} \mathrm{H}$ NMR $\delta 0.13(\mathrm{~s}, 3 \mathrm{H}), 0.14(\mathrm{~s}, 3 \mathrm{H}), 1.00(\mathrm{~s}, 9 \mathrm{H}), 2.18(\mathrm{~s}, 3 \mathrm{H}), 3.63\left(\mathrm{dd}, J_{\mathrm{AB}}=\right.$ $10.1, J=8.5 \mathrm{~Hz}, 1 \mathrm{H}), 4.00\left(\mathrm{dd}, J_{\mathrm{AB}}=10.1, J=4.5 \mathrm{~Hz}, 1 \mathrm{H}\right), 4.91(\mathrm{dd}, J=8.5,4.5 \mathrm{~Hz}, 1 \mathrm{H})$, 7.30-7.50 (m, 5H); ${ }^{13} \mathrm{C}$ NMR $\delta-5.9,-5.7,13.0,18.0,25.6,66.7,68.6,119.8$ (q, $\left.J=278.4\right)$, 127.3, 127.5, 128.4, 139.1, $156.0(\mathrm{q}, J=33.2 \mathrm{~Hz}) ;{ }^{19} \mathrm{~F}$ NMR $\delta-75.1(\mathrm{~s}) ;$ MS (EI $\left.70 \mathrm{eV}\right) \mathrm{m} / \mathrm{z}$ $345\left(\mathrm{M}^{+}\right), 288,200,177,118(100), 89,77$. 


\section{(E)-2,2,2-Trifluoro-1-methylethylidene ((1R)-2-diphenylmethylsilyloxy-1-phenylethyl)-}

amine (1g). Following the same procedure than for the preparation of $\mathbf{1 f}$, the compound $\mathbf{1 g}$ was obtained from imine 1e $(230 \mathrm{~g}, 1.0 \mathrm{mmol})$ and DPMSCl $(0.25 \mathrm{~mL}, 1.2 \mathrm{mmol})$. Purification by flash chromatography (97:3 petroleum ether/ethyl acetate) gave $\mathbf{1 g}$ (184 mg, $50 \%$ ) as a pale yellow oil. $[\alpha]^{25}-4.8\left(c\right.$ 1.8, $\mathrm{CHCl}_{3}$ ); IR (neat) 3070, 2924, 1692, 1429, 1201, 1121. $\mathrm{cm}^{-1} ;{ }^{1} \mathrm{H}$ NMR $\delta 0.82(\mathrm{~s}, 3 \mathrm{H}), 2.21(\mathrm{~s}, 3 \mathrm{H}), 4.18(\mathrm{~d}, J=6.4 \mathrm{~Hz}, 2 \mathrm{H}), 5.02(\mathrm{t}, J=6.4 \mathrm{~Hz}$, 1H), 7.30-7.60 (m, 10H), 7.70-7.90 (m, 5H); ${ }^{13} \mathrm{C}$ NMR $\delta-3.4,13.3,66.7,68.7,119.9(\mathrm{q}, J=$ $278.5 \mathrm{~Hz}), 127.4,127.7,127.9,128.6,129.6,129.9,133.9,134.3,137.5,139.0,156.7$ (q, $J=$ $33.1 \mathrm{~Hz}) ;{ }^{19} \mathrm{~F}$ NMR $\delta$-75.0 (s); MS (EI $\left.70 \mathrm{eV}\right) \mathrm{m} / \mathrm{z} 427\left(\mathrm{M}^{+}\right), 350,227,197(100), 103,90$, 77.

Representative Procedure for the Preparation of aminonitriles (2a-g) from imines (1ag): 3,3,3-Trifluoro-2-((S)-1-phenylethylamino)-propionitrile (2a). To a solution of imine 1a (200 mg, $1.0 \mathrm{mmol})$ in dichloromethane $(5 \mathrm{~mL})$ under argon was added cyanotrimethylsilane $(0.14 \mathrm{~mL}, 1.5 \mathrm{mmol})$ and $\mathrm{Yb}(\mathrm{OTf}) 3(124 \mathrm{mg}, 0.2 \mathrm{mmol})$ at $0{ }^{\circ} \mathrm{C}$. The reaction mixture was stirred at room temperature until disappearance of the starting material (2-4 days, GC monitoring). The reaction mixture was then poured into a saturated aqueous solution of $\mathrm{NaHCO}_{3}(20 \mathrm{~mL})$. The aqueous layer was extracted with dichloromethane $(3 \mathrm{x} 10$ $\mathrm{mL}$ ) and the combined organic extracts were dried over $\mathrm{Na}_{2} \mathrm{SO}_{4}$, filtered, and concentrated under reduced pressure. Purification by flash chromatography (19:1 petroleum ether/ethyl acetate) gave two pure isolated fractions (91 $\mathrm{mg}, 40 \%)$ and (91 $\mathrm{mg}, 40 \%$ ) of each diastereomers of 2a. $2 \mathbf{a}_{\text {diast } 1}$ : colorless oil. ; $[\alpha]^{20}{ }_{\mathrm{D}}+192.6$ (c 1.4, $\mathrm{CHCl}_{3}$ ); IR (neat) 3334, 2972, 1605, $1451 \mathrm{~cm}^{-1} ;{ }^{1} \mathrm{H}$ NMR $\delta 1.37(\mathrm{~d}, J=6.8 \mathrm{~Hz}, 3 \mathrm{H}), 1.92(\mathrm{~d}, J=13.1 \mathrm{~Hz}, 1 \mathrm{H}), 3.71$ $(\mathrm{dq}, J=13.1,7.2 \mathrm{~Hz}, 1 \mathrm{H}), 4.02(\mathrm{q}, J=6.8 \mathrm{~Hz}, 1 \mathrm{H}), 7.20-7.40(\mathrm{~m}, 5 \mathrm{H}) ;{ }^{13} \mathrm{C}$ NMR $24.7,51.1$ 
$(\mathrm{q}, J=34.4 \mathrm{~Hz}), 56.2,113.6,121.6(\mathrm{q}, J=279.9 \mathrm{~Hz}), 126.8,128.3,129.1,141.2 ;{ }^{19} \mathrm{~F} \mathrm{NMR} \delta-$

$74.5(\mathrm{~d}, J=7.2 \mathrm{~Hz})$; MS (EI $70 \mathrm{eV}) \mathrm{m} / z 228\left(\mathrm{M}^{+}\right), 213(100), 202,151,135,105,77$. Anal.

Calcd for $\mathrm{C}_{11} \mathrm{H}_{11} \mathrm{~F}_{3} \mathrm{~N}_{2}$ : C, 57.89; H, 4.86; N, 12.28. Found: C, 58.29; H, 4.92; N, 11.86.

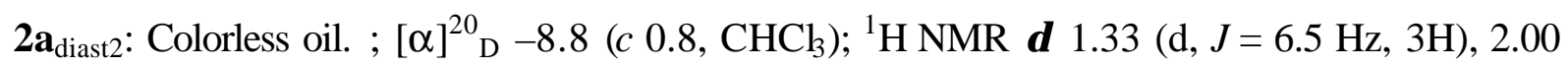
$(\mathrm{d}, J=12.0 \mathrm{~Hz}, 1 \mathrm{H}), 3.97(\mathrm{dq}, J=12.0,6.3 \mathrm{~Hz}, 1 \mathrm{H}), 3.99(\mathrm{q}, J=6.5 \mathrm{~Hz}, 1 \mathrm{H}), 7.10-7.40(\mathrm{~m}$, $5 \mathrm{H}) ;{ }^{13} \mathrm{C}$ NMR $22.8,50.6(\mathrm{q}, J=34.4 \mathrm{~Hz}), 56.5,113.5,122.1(\mathrm{q}, J=282.4 \mathrm{~Hz}), 126.7,128.2$, 129.0, $142.2 ;{ }^{19} \mathrm{~F}$ NMR $\delta-74.2(\mathrm{~d}, J=6.3 \mathrm{~Hz})$.

3,3,3-Trifluoro-2-methyl-2-((S)-1-phenylethylamino)-propionitrile $\quad$ (2b). $\quad$ Starting from imine $\mathbf{1 b}(210 \mathrm{mg}, 1.0 \mathrm{mmol})$, following the same procedure as for $\mathbf{2 a}$, pure isolated fractions (145 $\mathrm{mg}, 63 \%)$ and (40 $\mathrm{mg}, 17 \%)$ of each diastereomers $\mathbf{2 b}$ were obtained after purification by flash chromatography (19:1 petroleum ether/ethyl acetate). $\mathbf{2 b}_{\text {major: }}$ white solid. Mp $58{ }^{\circ} \mathrm{C}$; $[\alpha]^{20}{ }_{\mathrm{D}}+112.0\left(c\right.$ 1.5, $\left.\mathrm{CHCl}_{3}\right) ; \mathrm{IR}(\mathrm{KBr}) 3360,3030,2973,2361,1454,1174 \mathrm{~cm}^{-1} ;{ }^{1} \mathrm{H} \mathrm{NMR} \delta$ $1.21(\mathrm{~s}, 3 \mathrm{H}), 1.34(\mathrm{~d}, J=6.8 \mathrm{~Hz}, 3 \mathrm{H}), 1.85(\mathrm{~s}, 1 \mathrm{H}), 4.05(\mathrm{q}, J=6.8 \mathrm{~Hz}, 1 \mathrm{H}), 7.00-7.40(\mathrm{~m}$, $5 \mathrm{H}) ;{ }^{13} \mathrm{C}$ NMR 20.0, 26.8, 54.7, $59.5(\mathrm{q}, J=30.1 \mathrm{~Hz}), 116.5,123.6(\mathrm{q}, J=283.7 \mathrm{~Hz}), 126.1$, 127.3, 128.6, 145.5; ${ }^{19} \mathrm{~F}$ NMR $\delta-80.0(\mathrm{~s})$; MS (EI $\left.70 \mathrm{eV}\right) \mathrm{m} / z 242\left(\mathrm{M}^{+}\right), 227,216,200,165$, 122, 105 (100). $2 \mathbf{b}_{\text {minor: }}$ pale yellow oil. $[\alpha]^{20}{ }_{\mathrm{D}}+47.3\left(c 0.9, \mathrm{CHCl}_{3}\right) ;{ }^{1} \mathrm{H}$ NMR $\delta 1.39(\mathrm{~d}, J=$ $6.7 \mathrm{~Hz}, 3 \mathrm{H}), 1.61(\mathrm{~s}, 3 \mathrm{H}), 4.05(\mathrm{q}, J=6.7 \mathrm{~Hz}, 1 \mathrm{H}), 7.10-7.40(\mathrm{~m}, 5 \mathrm{H}) ;{ }^{13} \mathrm{C}$ NMR 20.0, 25.5, $54.4,58.0(\mathrm{q}, J=30.1 \mathrm{~Hz}), 116.3,123.8(\mathrm{q}, J=283.7 \mathrm{~Hz}), 126.1,127.5,128.6,143.9 ;{ }^{19} \mathrm{~F}$ NMR $\delta-79.2(\mathrm{~s})$.

3,3,3-Trifluoro-2-phenyl-2-((S)-1-phenylethylamino)-propionitrile $\quad$ (2c). $\quad$ Starting from imine 1c $(280 \mathrm{mg}, 1.0 \mathrm{mmol})$, following the same procedure as for $\mathbf{2 a}$, pure isolated fractions (205 mg, 67\%) and (68 mg, 23\%) of each diastereomers $2 \mathbf{c}$ were obtained after purification by flash chromatography (19:1 petroleum ether/ethyl acetate). 2c was obtained in 93\% yield as a 
67:33 diastereomeric mixture when the reaction was performed with $\operatorname{MgBr}_{2}$ (380 mg, 1.5

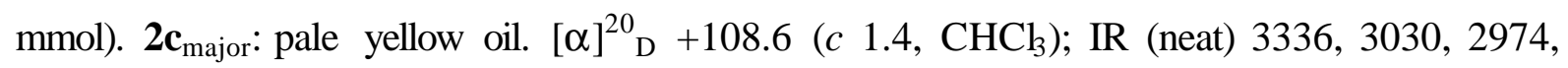
1963, 1493, 1452, $1174 \mathrm{~cm}^{-1} ;{ }^{1} \mathrm{H}$ NMR $\delta 1.61(\mathrm{~d}, J=6.7 \mathrm{~Hz}, 3 \mathrm{H}), 2.35(\mathrm{~s}, 1 \mathrm{H}), 3.98(\mathrm{q}, J=$ $6.7 \mathrm{~Hz}, 1 \mathrm{H}), 7.10-7.40(\mathrm{~m}, 8 \mathrm{H}), 7.40-7.60(\mathrm{~m}, 2 \mathrm{H}) ;{ }^{13} \mathrm{C} \mathrm{NMR} 24.4,54.7,67.9$ (q, $J=29.6$ $\mathrm{Hz}), 116.3,122.5(\mathrm{q}, J=283.7 \mathrm{~Hz}), 126.3,126.9,128.2,128.3,128.4,129.0,130.3,144.5 ;{ }^{19} \mathrm{~F}$

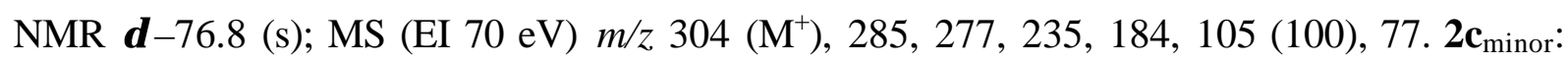
pale yellow oil. $[\alpha]^{20}{ }_{\mathrm{D}}+65.7\left(c 1.0, \mathrm{CHCl}_{3}\right) ;{ }^{1} \mathrm{H} \mathrm{NMR} \delta 1.35(\mathrm{~d}, J=6.8 \mathrm{~Hz}, 3 \mathrm{H}), 2.38(\mathrm{~s}, 1 \mathrm{H})$, $3.94(\mathrm{q}, J=6.8 \mathrm{~Hz}, 1 \mathrm{H}), 7.10-7.30(\mathrm{~m}, 6 \mathrm{H}), 7.40-7.60(\mathrm{~m}, 4 \mathrm{H}) ;{ }^{13} \mathrm{C}$ NMR 24.7, 54.7, $66.8(\mathrm{q}$, $J=29.6 \mathrm{~Hz}), 114.7,122.5(\mathrm{q}, J=285.3 \mathrm{~Hz}), 126.3,126.5,127.0,127.8,128.5,130.3,130.6$, $143.0 ;{ }^{19} \mathrm{~F}$ NMR $\delta-76.7(\mathrm{~s})$.

3,3,3-Trifluoro-2-((1R)-2-methoxy-1-phenylethylamino)-propionitrile $\quad$ (2d). Starting from imine $\mathbf{1 d}(230 \mathrm{mg}, 1.0 \mathrm{mmol})$, following the same procedure as for $\mathbf{2 c}$ with $\mathrm{MgBr}_{2}(380 \mathrm{mg}$, $1.5 \mathrm{mmol})$, pure isolated fractions (128 $\mathrm{mg}, 50 \%)$ and (32 $\mathrm{mg}, 12 \%)$ of each diastereomers $2 \mathrm{~d}$ were obtained after purification by flash chromatography (19:1 petroleum ether/ethyl acetate). 2d was obtained in 92\% yield as a 55:45 diastereomeric mixture when the reaction was performed with $\mathrm{Yb}(\mathrm{OTf})_{3}(124 \mathrm{mg}, 0.2 \mathrm{mmol})$. 2 $\mathbf{d}_{\text {major }}$ : pale yellow oil. $[\alpha]^{20}{ }_{\mathrm{D}}-176.2(c$ 1.1, $\mathrm{CHCl}_{3}$ ); IR (neat) 3337, 3031, 2933, 1604, 1456, 1349, $1150 \mathrm{~cm}^{-1} ;{ }^{1} \mathrm{H}$ NMR $\delta 2.85(\mathrm{~d}, J=$ $13.4 \mathrm{~Hz}, 1 \mathrm{H}), 3.45(\mathrm{dd}, J=9.7,9.5 \mathrm{~Hz}, 1 \mathrm{H}), 3.53(\mathrm{dd}, J=9.7,4.3 \mathrm{~Hz}, 1 \mathrm{H}), 3.86(\mathrm{~s}, 3 \mathrm{H}), 3.89$ $(\mathrm{dq}, J=13.4,6.3 \mathrm{~Hz}, 1 \mathrm{H}), 4.24(\mathrm{dd}, J=9.5,4.3 \mathrm{~Hz}, 1 \mathrm{H}), 7.30-7.50(\mathrm{~m}, 5 \mathrm{H}) ;{ }^{13} \mathrm{C}$ NMR 50.8 $(\mathrm{q}, J=34.4 \mathrm{~Hz}), 58.6,59.9,76.4,113.3,121.6(\mathrm{q}, J=280.0 \mathrm{~Hz}), 127.6,128.9,129.1,136.2$; ${ }^{19}$ F NMR $\delta-74.3(\mathrm{~d}, J=6.3 \mathrm{~Hz}) ; \mathrm{MS}(\mathrm{EI} 70 \mathrm{eV}) \mathrm{m} / z 259\left(\mathrm{M}^{+}+1\right), 232,212(100), 186$, 135,103, 77. $2 \mathbf{d}_{\text {minor }}$ : pale yellow oil. $[\alpha]^{20}{ }_{\mathrm{D}}-2.8\left(c\right.$ 0.6, $\left.\mathrm{CHCl}_{3}\right) ;{ }^{1} \mathrm{H} \mathrm{NMR} \delta 2.63(\mathrm{~d}, J=11.8$ $\mathrm{Hz}, 1 \mathrm{H}), 3.43(\mathrm{~s}, 3 \mathrm{H}), 3.4-3.6(\mathrm{~m}, 2 \mathrm{H}), 4.27(\mathrm{dd}, J=8.7,3.8 \mathrm{~Hz}, 1 \mathrm{H}), 4.47(\mathrm{dq}, J=11.8,6.3$ 
$\mathrm{Hz}, 1 \mathrm{H}), 7.30-7.50(\mathrm{~m}, 5 \mathrm{H}) ;{ }^{13} \mathrm{C}$ NMR 50.9 (q, $\left.J=33.9 \mathrm{~Hz}\right), 58.9,60.0,77.8,113.8,122.3$ (q, $J=283.1 \mathrm{~Hz}), 127.5,128.7,129.0,137.5 ;{ }^{19} \mathrm{~F}$ NMR $\delta-73.8(\mathrm{~d}, J=6.3 \mathrm{~Hz})$.

\section{3,3,3-Trifluoro-2-((1R)-2-hydroxy-1-phenylethylamino)-2-methylpropionitrile}

(2e).

Starting from imine 1e $(1.15 \mathrm{~g}, 5.0 \mathrm{mmol})$, following the same procedure as for $\mathbf{2 a}$, pure isolated fractions (620 mg, 48\%) and (550 $\mathrm{mg}, 43 \%)$ of each diastereomers $2 \mathrm{e}$ were obtained after purification by flash chromatography (85:15 petroleum ether/ethyl acetate).

\section{3,3,3-Trifluoro-2-((1R)-2-tert-Butyldimethylsilyloxy-1-phenylethylamino)-2-}

methylpropionitrile (2f). Starting from imine 1f $(350 \mathrm{mg}, 1 \mathrm{mmol})$, following the same procedure as for $2 \mathbf{a}$, $2 \mathbf{2}(280 \mathrm{mg}, 75 \%)$ was obtained as a $75: 25$ diastereomeric mixture after purification by flash chromatography (98:2 petroleum ether/ethyl acetate). $2 \mathbf{f}$ was obtained in 93\% yield as a 68:32 diastereomeric mixture when the reaction was performed with $\mathrm{MgBr}_{2}$

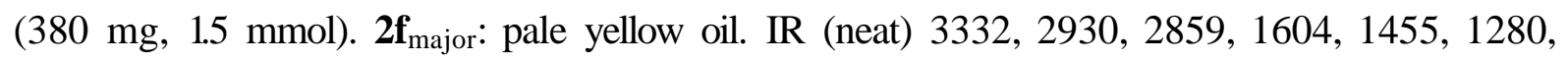
$1176 \mathrm{~cm}^{-1} ;{ }^{1} \mathrm{H}$ NMR $\delta 0.01$ (s, 3H), 0.02 (s, 3H), 0.85 (s, 9H), 1.13 (s, 3H), $1.60(\mathrm{~s}, 1 \mathrm{H}), 3.30$ (dd, $J=10.4,9.7 \mathrm{~Hz}, 1 \mathrm{H}), 3.58(\mathrm{dd}, J=10.4,4.2 \mathrm{~Hz}, 1 \mathrm{H}), 3.95(\mathrm{dd}, J=9.7,4.2 \mathrm{~Hz}, 1 \mathrm{H})$, 7.10-7.40 (m, 5H); ${ }^{13} \mathrm{C}$ NMR -5.7, -5.5, 17.9 , 20.2, 60.3 (q, $J=29.9$ Hz), 61.3, 67.2, 116.2, $123.2(\mathrm{q}, J=283.1 \mathrm{~Hz}), 127.2,127.9,128.6,140.7 ;{ }^{19} \mathrm{~F}$ NMR $\delta-80.2(\mathrm{~s}) ; \mathrm{MS}(\mathrm{EI} 70 \mathrm{eV}) \mathrm{m} / \mathrm{z}$ $372\left(\mathrm{M}^{+}\right), 346,288,227,200,177$ (100), 118, 89, 73. Anal. Calcd for $\mathrm{C}_{18} \mathrm{H}_{27} \mathrm{~F}_{3} \mathrm{~N}_{2} \mathrm{OSi}$ : C, 58.04; H, 7.31; N, 7.52. Found: C, 58.10; H, 7.64; N, 7.71. $2 \mathbf{2}_{\text {minor }}$ : pale yellow oil. ${ }^{1} \mathrm{H}$ NMR $\delta-0.02$ (s, 3H), -0.01 (s, 3H), 0.82 (s, 9H), 1.18 (s, 3H), 1.50 (s, 1H), 3.44 (dd, $J=10.3,8.5$ $\mathrm{Hz}, 1 \mathrm{H}), 3.75(\mathrm{dd}, J=10.3,4.4 \mathrm{~Hz}, 1 \mathrm{H}), 4.13(\mathrm{dd}, J=8.8,4.4 \mathrm{~Hz}, 1 \mathrm{H}), 7.20-7.40(\mathrm{~m}, 5 \mathrm{H})$; ${ }^{13} \mathrm{C}$ NMR $-5.7,-5.5,18.1,21.0,58.6(\mathrm{q}, J=31.2 \mathrm{~Hz}), 61.5,67.4,115.9,123.2(\mathrm{q}, J=284.9$ $\mathrm{Hz}), 127.6,128.0,128.2,140.0 ;{ }^{19} \mathrm{~F}$ NMR $\delta-78.1(\mathrm{~s})$. 


\section{3,3,3-Trifluoro-2-methyl-2-((1R)-2-methyldiphenylsilyloxy-1-phenylethylamino)-}

propionitrile $(\mathbf{2 g})$. Starting from imine $1 \mathrm{~g}(430 \mathrm{mg}, 1.0 \mathrm{mmol})$, following the same procedure as for $\mathbf{2 a}$, pure isolated fractions $(253 \mathrm{mg}, 56 \%)$ and $(170 \mathrm{mg}, 37 \%)$ of each diastereomers $\mathbf{2 g}$ were obtained after purification by flash chromatography (97:3 petroleum ether/ethyl acetate). 2gmajor: pale yellow oil. $[\alpha]^{20}{ }_{D}-39.6$ (c 0.9, $\mathrm{CHCl}_{3}$ ); IR (neat) 3351, 3070, 2926, 1725, 1429, $1120 \mathrm{~cm}^{-1} ;{ }^{1} \mathrm{H}$ NMR $\delta 0.59$ (s, 3H), 1.15 (s, 3H), 2.99 (s, 1H), 3.44 (t, $\left.J=10.1 \mathrm{~Hz}, 1 \mathrm{H}\right), 3.66$ $(\mathrm{dd}, J=10.1,4.1 \mathrm{~Hz}, 1 \mathrm{H}), 4.00(\mathrm{dd}, J=10.1,4.1 \mathrm{~Hz}, 1 \mathrm{H}), 7.10-7.30(\mathrm{~m}, 11 \mathrm{H}), 7.40-7.60(\mathrm{~m}$, $4 \mathrm{H}) ;{ }^{13} \mathrm{C}$ NMR $-3.3,20.9,58.6(\mathrm{q}, J=31.2 \mathrm{~Hz}), 61.3,67.6,116.6,123.1(\mathrm{q}, J=285.3 \mathrm{~Hz})$, $127.2,127.7,128.0,128.3,130.1,134.3,134.3,135.0,135.1,139.8 ;{ }^{19} \mathrm{~F}$ NMR $\delta-80.1$ (s); MS (EI $70 \mathrm{eV}) \mathrm{m} / z 454\left(\mathrm{M}^{+}\right), 427,350,227,197(100), 181,113,91.2 \mathbf{g}_{\text {minor: }}$ pale yellow oil. $[\alpha]^{20}{ }_{\mathrm{D}}-44.7\left(c \mathrm{c} 0.3, \mathrm{CHCl}_{3}\right) ;{ }^{1} \mathrm{H}$ NMR $\delta 0.61(\mathrm{~s}, 3 \mathrm{H}), 1.60(\mathrm{~s}, 3 \mathrm{H}), 2.51(\mathrm{~d}, J=3.0 \mathrm{~Hz}, 1 \mathrm{H})$, $3.56(\mathrm{dd}, J=10.5,8.3 \mathrm{~Hz}, 1 \mathrm{H}), 3.82(\mathrm{dd}, J=10.5,4.0 \mathrm{~Hz}, 1 \mathrm{H}), 4.13(\mathrm{ddd}, J=8.3,4.0,3.0 \mathrm{~Hz}$, 1H), 7.20-7.40 (m, 15H); ${ }^{13} \mathrm{C}$ NMR -3.3, $20.9,58.6$ (q, $\left.J=31.2 \mathrm{~Hz}\right), 61.3,67.6,116.6,123.1$ $(\mathrm{q}, J=285.3 \mathrm{~Hz}), 127.2,127.7,128.0,128.3,130.1,134.3,134.3,135.0,135.1,139.8 ;{ }^{19} \mathrm{~F}$ NMR $\delta-78.2(\mathrm{~s})$.

\section{Preparation of aminonitriles $(4 \mathbf{a}, \mathbf{b})$ from silylhemiacetals $(3 \mathbf{a}, \mathbf{b})$.}

Dibenzyl-(2,2,2-trifluoro-1-trimethylsilyloxyethyl)-amine (3a). To a solution of dibenzylamine $(890 \mathrm{mg}, 4.5 \mathrm{mmol})$ in $\mathrm{CH}_{2} \mathrm{Cl}_{2}(5 \mathrm{~mL})$ was added sodium sulfate and trifluoroacetaldehyde methyl hemiacetal $(650 \mathrm{mg}, 4.95 \mathrm{mmol})$. The mixture was stirred for 48 $\mathrm{h}$ at room temperature. The crude mixture was then filtered and concentrated under reduced pressure. The resulting hemiaminal $(1.30 \mathrm{~g}, 98 \%)$ as a pale yellow oil was used in the next step without purification. ${ }^{1} \mathrm{H}$ NMR $\delta 3.87(\mathrm{~d}, J=14 \mathrm{~Hz}, 2 \mathrm{H}), 4.13(\mathrm{~d}, J=14 \mathrm{~Hz}, 2 \mathrm{H}), 4.75$ (q, $J=6.0 \mathrm{~Hz}, 1 \mathrm{H}), 7.30-7.60(\mathrm{~m}, 10 \mathrm{H}) ;{ }^{13} \mathrm{C} \mathrm{NMR} \delta 52.8,80.2(\mathrm{q}, J=32.8 \mathrm{~Hz}), 123.9(\mathrm{q}, J=$ 
$285.5 \mathrm{~Hz}), 127.3,128.6,130.8,138.5 ;{ }^{19} \mathrm{~F}$ NMR $\delta-76.8(\mathrm{~d}, J=6.0 \mathrm{~Hz})$; MS $(\mathrm{EI} 70 \mathrm{eV}) \mathrm{m} / z$ $295\left(\mathrm{M}^{+}\right), 196,120,106,91(100)$.

The hemiaminal $(1.30 \mathrm{~g}, 4.4 \mathrm{mmol})$ was then stirred with $N$-trimethylsilylimidazole $(0.65 \mathrm{~g}$, $4.4 \mathrm{mmol})$ in THF $(5 \mathrm{~mL})$ for $20 \mathrm{~min}$ at $0{ }^{\circ} \mathrm{C}$ and $5 \mathrm{~h}$ at room temperature. The mixture was treated with pentane $(20 \mathrm{~mL})$ and a saturated $\mathrm{NaHCO}_{3}$ solution. The organic layer was washed twice with a saturated $\mathrm{NaHCO}_{3}$ solution, dried over $\mathrm{Na}_{2} \mathrm{SO}_{4}$ and filtered. The solvent was removed under reduced pressure to give 3a as a pale yellow oil. IR (neat) 3087, 3029, 2958, 2851, 1495, 1454, 1255, 1177. $\mathrm{cm}^{-1} ;{ }^{1} \mathrm{H}$ NMR $\delta 0.11(\mathrm{~s}, 9 \mathrm{H}), 3.59(\mathrm{~d}, J=14.2 \mathrm{~Hz}, 2 \mathrm{H})$, $3.98(\mathrm{~d}, J=14.2 \mathrm{~Hz}, 2 \mathrm{H}), 4.52(\mathrm{q}, J=6.3 \mathrm{~Hz}, 1 \mathrm{H}), 7.20-7.50(\mathrm{~m}, 10 \mathrm{H}) ;{ }^{13} \mathrm{C} \mathrm{NMR}$ $\delta$ 0.02, 53.2, $80.9(\mathrm{q}, J=32.6 \mathrm{~Hz}), 123.9(\mathrm{q}, J=285.9 \mathrm{~Hz}), 126.2,127.2,128.1,128.3,128.6$, 130.7, 138.6, 139.8; ${ }^{19} \mathrm{~F}$ NMR $\delta-77.1(\mathrm{~d}, J=6.3 \mathrm{~Hz})$; MS (EI $\left.70 \mathrm{eV}\right) \mathrm{m} / \mathrm{z} 368\left(\mathrm{M}^{+}+1\right), 352$, $348,296,278,181,134,73(100)$.

\section{2-Dibenzylamino-3,3,3-trifluoropropionitrile (4a).}

To a solution of silylhemiacetal $\mathbf{3 a}(370 \mathrm{mg}, 1 \mathrm{mmol})$ in dichloromethane $(5 \mathrm{~mL})$ at $0{ }^{\circ} \mathrm{C}$ under argon was added cyanotrimethylsilane $(0.20 \mathrm{~mL}, 1.5 \mathrm{mmol})$ and $\mathrm{BF}_{3} \cdot \mathrm{OEt}_{2}(0.19 \mathrm{~mL}$, $1.5 \mathrm{mmol}$ ). The reaction mixture was stirred at room temperature overnight. The mixture was then poured into a saturated aqueous solution of $\mathrm{NaHCO} 3(20 \mathrm{~mL})$. The aqueous layer was extracted with dichloromethane $(3 \times 20 \mathrm{~mL})$, and the combined organic extracts were dried over $\mathrm{Na}_{2} \mathrm{SO}_{4}$, filtered, and concentrated under reduced pressure. Purification by flash chromatography (19:1 petroleum ether/ethyl acetate) gave pure $\mathbf{4 a}(277 \mathrm{mg}, 91 \%)$ as a pale yellow oil. IR (neat) 3032, 2926, 1604, 1361, $1196 \mathrm{~cm}^{-1} ;{ }^{1} \mathrm{H}$ NMR $\delta 3.70(\mathrm{~d}, J=13.7 \mathrm{~Hz}$, $2 \mathrm{H}), 4.24(\mathrm{~d}, J=13.7 \mathrm{~Hz}, 2 \mathrm{H}), 4.39(\mathrm{q}, J=7.2 \mathrm{~Hz}, 1 \mathrm{H}), 7.40-7.60(\mathrm{~m}, 10 \mathrm{H}) ;{ }^{13} \mathrm{C}$ NMR 54.8 $(\mathrm{q}, J=34.0 \mathrm{~Hz}), 56.1,110.1(\mathrm{q}, J=1.8 \mathrm{~Hz}), 122.1(\mathrm{q}, J=284.1 \mathrm{~Hz}), 128.0,128.6,128.7$, 
136.1; ${ }^{19} \mathrm{~F}$ NMR $\delta-70.4(\mathrm{~d}, J=7.2 \mathrm{~Hz})$; MS (EI $\left.70 \mathrm{eV}\right) \mathrm{m} / z .304\left(\mathrm{M}^{+}\right), 278,235,213,91$ $(100)$.

2-Dimethylamino-2-phenyl-3,3,3-trifluoropropionitrile (4b). Under argon atmosphere, a mixture of trifluoroacetophenone $(440 \mathrm{mg}, 2.5 \mathrm{mmol})$ and $N$-trimethylsilyldimethylamine was heated at $110{ }^{\circ} \mathrm{C}$ in a sealed tube for $24 \mathrm{~h}$. The mixture was cooled down to $0{ }^{\circ} \mathrm{C}$, and dichloromethane $(5 \mathrm{~mL})$, cyanotrimethylsilane $(0.5 \mathrm{~mL}, 3.75 \mathrm{mmol})$ and $\mathrm{BF}_{3} \cdot \mathrm{OEt}_{2}(0.48 \mathrm{~mL}$, $3.75 \mathrm{mmol}$ ) were added. The reaction mixture was stirred at room temperature for $24 \mathrm{~h}$. The mixture was then poured into a saturated aqueous solution of $\mathrm{NaHCO}_{3}(20 \mathrm{~mL})$. The aqueous layer was extracted with dichloromethane $(3 \times 20 \mathrm{~mL})$, and the combined organic extracts were dried over $\mathrm{Na}_{2} \mathrm{SO}_{4}$, filtered, and concentrated under reduced pressure. Purification by flash chromatography (97:3 petroleum ether/ethyl acetate) gave pure $\mathbf{4 b}(496 \mathrm{mg}, 87 \%$ ) as a pale yellow oil. IR (neat) 3030, 2973, 2847, 1452, $1172 \mathrm{~cm}^{-1} ;{ }^{1} \mathrm{H}$ NMR $\delta 2.38$ (s, 6H), 7.46 (m, 3H), $7.72(\mathrm{~m}, 2 \mathrm{H}) ;{ }^{13} \mathrm{C}$ NMR 41.7, $74.9(\mathrm{q}, J=29.0 \mathrm{~Hz}), 116.7,122.4(\mathrm{q}, J=286.3 \mathrm{~Hz})$, 127.7, 128.9, 130.3, 132.1; ${ }^{19}$ F NMR $\delta$-68.5 (s); MS (EI $\left.70 \mathrm{eV}\right) \mathrm{m} / \mathrm{z} 228\left(\mathrm{M}^{+}\right), 209,202,184$, $159(100), 151,154,77$.

Preparation of oxazolidines (5a-c) and (7a-e).

Oxazolidines $\mathbf{5} \mathbf{a}^{3}$ and $\mathbf{7} \mathbf{a}^{4}$ were prepared according literature procedures.

(4S,5R)-2-Trifluoromethyl-4-methyl-5-phenyl-1,3-oxazolidines (5b). To a stirred solution of $670 \mathrm{mg}$ of trifluoroacetaldehyde methyl hemiacetal $(4.3 \mathrm{mmol}, 1.0$ equiv) in $20 \mathrm{~mL}$ of

(3) Billard, T.; Langlois, B. R. J. Org. Chem. 2002, 67, 997-1000.

(4) (a) Ishii, A.; Higashiyama, K.; Mikami, K. Synlett 1997, 1381-1382. (b) Huguenot, F. Brigaud, T. J. Org. Chem. 2006, 71, 2159-2162 
toluene at room temperature, were added $650 \mathrm{mg}$ of $(1 R, 2 S)$-norephedrine $(4.3 \mathrm{mmol}, 1.0$ equiv) and $108 \mathrm{mg}$ of pyridinium $p$-toluenesulfonate ( $0.43 \mathrm{mmol}, 0.1$ equiv). The mixture was warmed to reflux with a Dean-Stark apparatus for $12 \mathrm{~h}$, then cooled to $0{ }^{\circ} \mathrm{C}$ with an ice-bath. The resulting mixture was filtered and toluene was evaporated. Purification by flash chromatography (19:1 petroleum ether /ethyl acetate) gave $940 \mathrm{mg}(95 \%)$ of an oil as a 85:15 mixture of diasteromers. $\mathbf{5 b}_{\text {major }}$ : IR (neat) 3334, 3034, 2978, 1496, 1455, 1290, $1136 \mathrm{~cm}^{-1}$; ${ }^{1} \mathrm{H}$ NMR $\delta 0.59(\mathrm{~d}, J=4.6 \mathrm{~Hz}, 3 \mathrm{H}), 2.34(\mathrm{~s}, 1 \mathrm{H}), 3.64(\mathrm{dq}, J=6.8,4.6 \mathrm{~Hz}, 1 \mathrm{H}), 5.04(\mathrm{~d}, J=6.8$ $\mathrm{Hz}, 1 \mathrm{H}), 5.11\left(\mathrm{q}, J_{\mathrm{HF}}=6.3 \mathrm{~Hz}, 1 \mathrm{H}\right), 7.10-7.30(\mathrm{~m}, 5 \mathrm{H}) ;{ }^{13} \mathrm{C} \mathrm{NMR} \delta 16.3,56.4,82.3,87.8(\mathrm{q}$, $J=34.0 \mathrm{~Hz}), 123.4(\mathrm{q}, J=283.5 \mathrm{~Hz}), 125.8,128.2,127.7,138.3 ;{ }^{19} \mathrm{~F} \mathrm{NMR} \delta-81.3(\mathrm{~d}, J=6.3$ $\mathrm{Hz})$; MS (EI $70 \mathrm{eV}) \mathrm{m} / \mathrm{z} 231\left(\mathrm{M}^{+}+1\right), 188,162,125(100), 105,77.5 \mathbf{b}_{\text {minor }}{ }^{1} \mathrm{H}$ NMR $\delta 0.82$ $(\mathrm{d}, J=6.8 \mathrm{~Hz}, 3 \mathrm{H}), 2.70(\mathrm{~s}, 1 \mathrm{H}), 3.71(\mathrm{dq}, J=6.9,6.8 \mathrm{~Hz}, 1 \mathrm{H}), 4.99\left(\mathrm{q}, J_{\mathrm{HF}}=6.3 \mathrm{~Hz}, 1 \mathrm{H}\right)$, $5.09(\mathrm{~d}, J=6.9 \mathrm{~Hz}, 1 \mathrm{H}), 7.20-7.50(\mathrm{~m}, 5 \mathrm{H}) ;{ }^{13} \mathrm{C} \mathrm{NMR} \delta 16.3,56.4,82.3,87.8(\mathrm{q}, J=34.0$ $\mathrm{Hz}), 123.4(\mathrm{q}, J=283.5 \mathrm{~Hz}), 125.8,128.2,127.7,138.3 ;{ }^{19} \mathrm{~F} \mathrm{NMR} \delta-80.1(\mathrm{~d}, J=6.3 \mathrm{~Hz})$.

(4S,5R)-2-Trifluoromethyl-2,4-dimethyl-5-phenyl-1,3-oxazolidines (5c). To a solution of trifluoroacetone $(530 \mathrm{mg}, 4.8 \mathrm{mmol})$ in $\mathrm{CH}_{2} \mathrm{Cl}_{2}(10 \mathrm{~mL})$ at $0{ }^{\circ} \mathrm{C}$ was added $(1 R, 2 S)$ norephedrine $(600 \mathrm{mg}, 4.0 \mathrm{mmol})$ and $\mathrm{BF}_{3} \cdot \mathrm{OEt}_{2}(0.6 \mathrm{~mL}, 4.8 \mathrm{mmol})$. After $48 \mathrm{~h}$ stirring at room temperature, the reaction mixture was quenched by addition of a saturated $\mathrm{NaHCO}_{3}$ solution $(10 \mathrm{~mL})$. The aqueous layer was extracted with $\mathrm{CH}_{2} \mathrm{Cl}_{2}(3 \mathrm{x} 20 \mathrm{~mL})$ and the combined organic extracts were dried over $\mathrm{Na}_{2} \mathrm{SO}_{4}$, filtered, and concentrated under reduced pressure. The crude product was purified by flash chromatography (19:1 petroleum ether lethyl acetate) to give pure fractions of $\mathbf{5} \mathbf{c}_{\text {major }}(64 \mathrm{mg}, 7 \%)$ and $\mathbf{5} \mathbf{c}_{\text {minor }}(45 \mathrm{mg}, 5 \%) . \mathbf{5} \mathbf{c}_{\text {major }}$ : yellow oil; $[\alpha]^{20}{ }_{\mathrm{D}}-74.0$ (c 1.1, $\mathrm{CHCl}_{3}$ ); IR (neat) 3330, 3032, 2931, 1455, $1164 \mathrm{~cm}^{-1} ;{ }^{1} \mathrm{H}$ NMR $\delta 0.66(\mathrm{~d}, J=6.9 \mathrm{~Hz}, 3 \mathrm{H}), 1.49(\mathrm{~s}, 3 \mathrm{H}), 2.45(\mathrm{~m}, 1 \mathrm{H}), 3.76(\mathrm{dq}, J=6.9,6.5 \mathrm{~Hz}, 1 \mathrm{H})$, $5.10(\mathrm{~d}, J=6.5 \mathrm{~Hz}, 1 \mathrm{H}), 7.10-7.40(\mathrm{~m}, 5 \mathrm{H}) ;{ }^{13} \mathrm{C} \mathrm{NMR} \delta 17.2,20.9,56.4,82.2,93.2(\mathrm{q}, J=$ 
$31.2 \mathrm{~Hz}), 123.9(\mathrm{q}, J=283.0 \mathrm{~Hz}), 126.2,127.7,128.2,137.0 ;{ }^{19} \mathrm{~F}$ NMR $\delta-82.7$ (s); MS (EI $70 \mathrm{eV}) \mathrm{m} / \mathrm{z} 246\left(\mathrm{M}^{+}+1\right), 227,201,176,158,139(100), 124,77 . \mathbf{5 c}_{\text {minor }}:$ Yellow oil; $[\alpha]^{20}{ }_{\mathrm{D}}-$ $35.6\left(c \quad 0.9, \mathrm{CHCl}_{3}\right) ;{ }^{1} \mathrm{H}$ NMR $\delta 0.63(\mathrm{~d}, J=6.6 \mathrm{~Hz}, 3 \mathrm{H}), 1.67(\mathrm{~s}, 3 \mathrm{H}), 1.93(\mathrm{~s}, 1 \mathrm{H}), 3.90(\mathrm{dq}$, $J=7.6,6.6 \mathrm{~Hz}, 1 \mathrm{H}), 5.20(\mathrm{~d}, J=7.6 \mathrm{~Hz}, 1 \mathrm{H}), 7.00-7.40(\mathrm{~m}, 5 \mathrm{H}) ;{ }^{13} \mathrm{C} \mathrm{NMR} \delta 17.3,20.8$, $57.4,83.5,93.5(\mathrm{q}, J=30.3 \mathrm{~Hz}), 125.0(\mathrm{q}, J=284.0 \mathrm{~Hz}), 126.2,127.6,128.2,138.7 ;{ }^{19} \mathrm{~F} \mathrm{NMR}$ $\delta-82.0(\mathrm{~s})$.

(4R)- $N$-Benzyl-2-trifluoromethyl-4-phenyl-1,3-oxazolidines $\quad(7 b) . \quad$ Following the same procedure than for the preparation of $\mathbf{5 b}$, starting from $(R)-N$-benzylphenylglycinol $(3.00 \mathrm{~g}$, $13.2 \mathrm{mmol})$, and trifluoroacetaldehyde methyl hemiacetal (1.81 g, $13.2 \mathrm{mmol})$, and after chromatography (19:1 petroleum ether /ethyl acetate), oxazolidines $7 \mathbf{b}$ (3.00 g, 74\%) were obtained as a 63:37 mixture of diastereomers. A pure analytical sample of each diastereomer

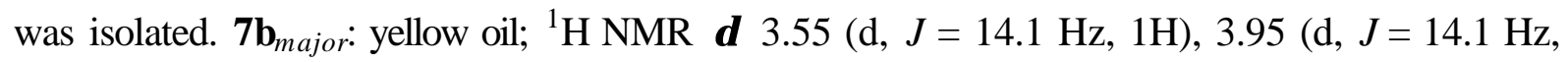
$1 \mathrm{H}), 4.11(\mathrm{dd}, J=7.3,3.0 \mathrm{~Hz}, 1 \mathrm{H}), 4.26(\mathrm{dd}, J=6.5,3.0 \mathrm{~Hz}, 1 \mathrm{H}), 4.50(\mathrm{dd}, J=7.3,6.5 \mathrm{~Hz}$, $1 \mathrm{H}), 4.91(\mathrm{q}, J=5.0 \mathrm{~Hz}, 1 \mathrm{H}), 7.10-7.40(\mathrm{~m}, 10 \mathrm{H}) ;{ }^{13} \mathrm{C} \mathrm{NMR} \delta 50.4,63.3,74.4,88.8(\mathrm{q}, J=$ $33.5 \mathrm{~Hz}), 123.7(\mathrm{q}, J=288.4 \mathrm{~Hz}), 127.1,128.0,128.1,128.3,128.7,129.0,137.8,138.4 ;{ }^{19} \mathrm{~F}$ NMR $\delta-79.4(\mathrm{~d}, J=5.0 \mathrm{~Hz}) .7 \mathbf{b}_{\text {minor }}$ : yellow oil; $[\alpha]^{20}{ }_{\mathrm{D}}-124.5\left(c \quad 0.6, \mathrm{CHCl}_{3}\right)$; IR (neat) 3087, 3065, 3030, 2895, 1495, 1456, 1293, $1142 \mathrm{~cm}^{-1} ;{ }^{1} \mathrm{H}$ NMR $\delta 3.76(\mathrm{dd}, J=16.4,7.6 \mathrm{~Hz}$, 1H), $3.82(\mathrm{~d}, J=14.2 \mathrm{~Hz}, 1 \mathrm{H}), 3.91(\mathrm{~d}, J=14.2 \mathrm{~Hz}, 1 \mathrm{H}), 4.24(\mathrm{dd}, J=16.4,6.5 \mathrm{~Hz}, 1 \mathrm{H}), 4.25$ $(\mathrm{dd}, J=7.6,6.5 \mathrm{~Hz}, 1 \mathrm{H}), 4.77(\mathrm{q}, J=4.6 \mathrm{~Hz}, 1 \mathrm{H}), 7.10-7.50(\mathrm{~m}, 10 \mathrm{H}) ;{ }^{13} \mathrm{C} \mathrm{NMR} \delta 55.0$, $65.5,74.9,89.7(\mathrm{q}, J=33.5 \mathrm{~Hz}), 124.1(\mathrm{q}, J=285.5 \mathrm{~Hz}), 127.6,127.7,128.0,128.3,128.6$, 129.7, 135.3, 137.3; ${ }^{19}$ F NMR $\delta-80.4(\mathrm{~d}, J=4.6 \mathrm{~Hz})$, MS (EI $\left.70 \mathrm{eV}\right) \mathrm{m} / z 307\left(\mathrm{M}^{+}\right), 238,160$, 117, 91 (100). 
(4R)- $N$-Benzoyl-2-trifluoromethyl-4-phenyl-1,3-oxazolidines $\quad$ (7c). To a solution of oxazolidine 7a $(1.00 \mathrm{~g}, 4.6 \mathrm{mmol})$ in $\mathrm{THF}(30 \mathrm{~mL})$ at $0{ }^{\circ} \mathrm{C}$, was added triethylamine $(1.28 \mathrm{~mL}$, $9.2 \mathrm{mmol})$ and benzoyle chloride $(1.1 \mathrm{~mL}, 9.2 \mathrm{mmol})$. The mixture was refluxed for $24 \mathrm{~h}$, and was filtered on celite. The crude mixture was then diluted with diethyl ether and washed with brine. The organic layer was dried over $\mathrm{Na}_{2} \mathrm{SO}_{4}$, filtered, and concentrated under reduced pressure. The crude product was purified by flash chromatography $9: 1$ petroleum ether /ethyl acetate), and was recrystallized in hexane to give pure 7c (990 mg, 50\%, single diastereomer) as a white solid. Mp 65-67 ${ }^{\circ} \mathrm{C}$; $[\alpha]^{20}{ }_{\mathrm{D}}-106.9$ (c 1.1, $\left.\mathrm{CHCl}_{3}\right)$; IR $(\mathrm{KBr}) 3067,3029,2978$, 2922, 1670, 1579, 1462, 1451, 1232, $1142 \mathrm{~cm}^{-1} ;{ }^{1} \mathrm{H}$ NMR $\delta 4.21(\mathrm{dd}, J=8.4,7.6 \mathrm{~Hz}, 1 \mathrm{H})$, $4.52(\mathrm{dd}, J=8.4,7.7 \mathrm{~Hz}, 1 \mathrm{H}), 5.13(\mathrm{dd}, J=7.7,7.6 \mathrm{~Hz}, 1 \mathrm{H}), 6.08(\mathrm{q}, J=5.5 \mathrm{~Hz}, 1 \mathrm{H}), 7.10$ $7.50(\mathrm{~m}, 10 \mathrm{H}) ;{ }^{13} \mathrm{C} \mathrm{NMR} \delta 62.3,73.2,86.0(\mathrm{q}, J=35.8 \mathrm{~Hz}), 123.0(\mathrm{q}, J=286.0 \mathrm{~Hz}), 125.3$, 126.3, 128.0, 128.0, 128.7, 130.3, 134.7, 135.3, 173.1; ${ }^{19} \mathrm{~F}$ NMR $\delta-76.4$ (d, $\left.J=5.5 \mathrm{~Hz}\right)$; MS (EI $70 \mathrm{eV}) \mathrm{m} / \mathrm{z} 321\left(\mathrm{M}^{+}+1\right), 223,221,216,140,105,77$ (100). Anal. Calcd for $\mathrm{C}_{17} \mathrm{H}_{14} \mathrm{~F}_{3} \mathrm{NO}_{2}$ : C, 63.55; H, 4.39; N, 4.36. Found: $\mathrm{C}, 63.91 ; \mathrm{H}, 4.32 ; \mathrm{N}, 4.21$.

(4R)-2-trifluoromethyl-2,4-diphenyl-1,3-oxazolidines (7d). Following the same procedure than for the preparation of $\mathbf{5 b}$, starting from $(R)$-phenylglycinol $(2.00 \mathrm{~g}, 14.6 \mathrm{mmol})$, and trifluorobenzophenone (2.60 g, $14.6 \mathrm{mmol})$, and after chromatography (97:3 petroleum ether /ethyl acetate), oxazolidines 7d (4.15 g, 97\%) were obtained as a 71:29 mixture of diastereomers. 7d $\mathbf{d}_{\text {major }}$ : IR (neat) 3378, 3052, 2991, 1459, 1424, $1060 \mathrm{~cm}^{-1} ;{ }^{1} \mathrm{H}$ NMR $\delta 2.60$ $(\mathrm{d}, J=10.1 \mathrm{~Hz}, 1 \mathrm{H}), 3.91(\mathrm{t}, J=8.7 \mathrm{~Hz}, 1 \mathrm{H}), 4.69(\mathrm{t}, J=8.7 \mathrm{~Hz}, 1 \mathrm{H}), 4.89(\mathrm{dt}, J=10.1,7.8$ $\mathrm{Hz}, 1 \mathrm{H}), 7.20-7.60(\mathrm{~m}, 8 \mathrm{H}), 7.85(\mathrm{~m}, 2 \mathrm{H}) ;{ }^{13} \mathrm{C} \mathrm{NMR} \delta 60.7,73.3,96.5(\mathrm{q}, J=30.1 \mathrm{~Hz})$, $122.1(\mathrm{q}, J=279.5 \mathrm{~Hz}), 126.1,126.7,127.9,128.3,128.6,129.4,136.7,137.7 ;{ }^{19} \mathrm{~F} \mathrm{NMR} \delta-$ 80.7 (s); MS (EI $70 \mathrm{eV}) \mathrm{m} / z 293\left(\mathrm{M}^{+}\right), 274,224,216,120$ (100), 77. Anal. Calcd for $\mathrm{C}_{16} \mathrm{H}_{14} \mathrm{~F}_{3} \mathrm{NO}: \mathrm{C}, 65.52 ; \mathrm{H}, 4.81 ; \mathrm{N}, 4.78$. Found: C, 65.84; H, 4.62; N, 5.01. 7d $\mathbf{d}_{\text {minor }}:{ }^{1} \mathrm{H} \mathrm{NMR}$ 
$\delta 3.16(\mathrm{~d}, J=7.7 \mathrm{~Hz}, 1 \mathrm{H}), 4.14(\mathrm{t}, J=10.5 \mathrm{~Hz}, 1 \mathrm{H}), 4.65(\mathrm{~m}, 2 \mathrm{H}), 7.20-7.60(\mathrm{~m}, 8 \mathrm{H}), 7.84$ $(\mathrm{m}, 2 \mathrm{H}) ;{ }^{13} \mathrm{C}$ NMR $\delta$ 62.6, 74.3, $95.0(\mathrm{q}, J=31.1 \mathrm{~Hz}), 122.0(\mathrm{q}, J=279.4 \mathrm{~Hz}), 126.4,126.7$, 128.0, 128.4, 128.8, 129.2, 136.1, 134.8; ${ }^{19} \mathrm{~F}$ NMR $\delta-80.3$ (s).

(4R)-2-trifluoromethyl-2-methyl-4-phenyl-1,3-oxazolidines $\quad(\mathbf{7 e}) . \quad$ Following the same procedure than for the preparation of $\mathbf{5 b}$, starting from $(R)$-phenylglycinol $(2.37 \mathrm{~g}, 17.3$ mmol), and trifluoroacetophenone (2.50 g, $17.3 \mathrm{mmol})$, and after chromatography (9:1 petroleum ether /ethyl acetate), oxazolidine $7 \mathrm{e}(2.5 \mathrm{~g}, 63 \%)$ was obtained as a single diastereomer as a yellow oil; $[\alpha]^{20}{ }_{\mathrm{D}}-23.2$ (c 1.8, $\mathrm{CHCl}_{3}$ ); IR (neat) 3356, 3033, 2999, 1458, 1338, $1156 \mathrm{~cm}^{-1} ;{ }^{1} \mathrm{H}$ NMR $\delta 1.63(\mathrm{~s}, 3 \mathrm{H}), 2.29(\mathrm{~d}, J=7.9 \mathrm{~Hz}, 1 \mathrm{H}), 3.82(\mathrm{t}, J=7.9 \mathrm{~Hz}, 1 \mathrm{H})$, $4.40(\mathrm{dd}, J=7.9,7.6 \mathrm{~Hz}, 1 \mathrm{H}), 4.59(\mathrm{dt}, J=7.9,7.6 \mathrm{~Hz}, 1 \mathrm{H}), 7.20-7.50(\mathrm{~m}, 5 \mathrm{H}) ;{ }^{13} \mathrm{C} \mathrm{NMR}$ $\delta$ 20.6, 62.0, 73.6, $94.2(\mathrm{q}, J=30.8 \mathrm{~Hz}), 124.8(\mathrm{q}, J=287.2 \mathrm{~Hz}), 126.7,128.2,128.9,138.8$; ${ }^{19}$ F NMR $\delta-82.9(\mathrm{~s})$; MS (EI $\left.70 \mathrm{eV}\right) \mathrm{m} / z 232\left(\mathrm{M}^{+}+1\right), 200,162(100), 132,120,77$. Anal. Calcd for $\mathrm{C}_{11} \mathrm{H}_{12} \mathrm{~F}_{3} \mathrm{NO}$ : C, 57.14; H, 5.23; N, 6.06. Found: C, 56.80; H, 5.05; N, 5.85.

Preparation of aminonitriles (6a-c, 8a,b,d) from oxazolidines (5a-c, 7a,b,d).

\section{3,3,3-Trifluoro-2-((1S,2R)-2-hydroxy-1-methyl-2-phenylethylmethylamino)-propionitrile}

(6a). Following the same procedure than for the synthesis of $2 \mathbf{e}$ (see experimental section), the aminonitrile 6a was prepared from the oxazolidine 5a (736 mg, $3 \mathrm{mmol}$ ). Purification by flash chromatography (9:1 petroleum ether/ethyl acetate) gave $\mathbf{6 a}(654 \mathrm{mg}, 80 \%)$ as a 63:37 mixture of diastereomers. 6a $\mathbf{a}_{\text {major }}$ IR (neat) $3485,2941,2886,1229,1121 \mathrm{~cm}^{-1} ;{ }^{1} \mathrm{H} \mathrm{NMR}$ $\delta 1.02(\mathrm{~d}, J=6.9 \mathrm{~Hz}, 3 \mathrm{H}), 2.04(\mathrm{~s}, 1 \mathrm{H}), 2.84(\mathrm{~s}, 3 \mathrm{H}), 2.62(\mathrm{~m}, 1 \mathrm{H}), 4.11(\mathrm{q}, J=7.2 \mathrm{~Hz}, 1 \mathrm{H})$, $5.20(\mathrm{~m}, 1 \mathrm{H}), 7.20-7.40(\mathrm{~m}, 5 \mathrm{H}) ;{ }^{13} \mathrm{C}$ NMR 6.2, 29.1, 54.4 (q, $\left.J=33.5 \mathrm{~Hz}\right), 64.9,71.0,112.3$, $122.8(\mathrm{q}, J=282.5 \mathrm{~Hz}), 125.0,125.4,127.7,142.4 ;{ }^{19} \mathrm{~F}$ NMR $\delta-72.3(\mathrm{~d}, J=7.2 \mathrm{~Hz}) . \mathbf{6} \mathbf{a}_{\text {minor }}$. ${ }^{1} \mathrm{H}$ NMR $\delta 1.06(\mathrm{~d}, J=7.3 \mathrm{~Hz}, 3 \mathrm{H}), 1.64(\mathrm{~s}, 1 \mathrm{H}), 2.64(\mathrm{~s}, 3 \mathrm{H}), 2.90(\mathrm{dq}, J=7.3,7.2 \mathrm{~Hz}, 1 \mathrm{H})$, 
$4.87(\mathrm{q}, J=7.2 \mathrm{~Hz}, 1 \mathrm{H}), 5.05(\mathrm{~d}, J=7.2 \mathrm{~Hz}, 1 \mathrm{H}), 7.30-7.40(\mathrm{~m}, 5 \mathrm{H}) ;{ }^{13} \mathrm{C} \mathrm{NMR} 10.7,38.6$, $54.0(\mathrm{q}, J=34.5 \mathrm{~Hz}), 65.3,77.8,112.4,122.2(\mathrm{q}, J=282.5 \mathrm{~Hz}), 125.0,127.7,128.4,142.2$; ${ }^{19}$ F NMR $\delta-73.2(\mathrm{~d}, J=7.2 \mathrm{~Hz})$.

\section{3,3,3-Trifluoro-2-((1S,2R)-2-hydroxy-1-methyl-2-phenylethylamino)-propionitrile}

(6b).

Following the same procedure than for the synthesis of $\mathbf{2 e}$ (see experimental section), the aminonitrile $\mathbf{6 b}$ was prepared from the oxazolidine $5 \mathbf{b}$ (230 $\mathrm{mg}, 1 \mathrm{mmol}$ ). Purification by flash chromatography (85:15 petroleum ether/ethyl acetate) gave $6 \mathbf{b}(232 \mathrm{mg}, 90 \%)$ as a 66:34 mixture of diastereomers. $\mathbf{6 b}_{\text {major }}$. IR (neat) 3345, 3035, 2978, 1605, 1453, 1241, 1152 $\mathrm{cm}^{-1} ;{ }^{1} \mathrm{H}$ NMR $\delta 0.85(\mathrm{~d}, J=6.6 \mathrm{~Hz}, 3 \mathrm{H}), 1.83(\mathrm{dd}, J=11.1,5.1 \mathrm{~Hz}, 1 \mathrm{H}), 2.80(\mathrm{~s}, 1 \mathrm{H}), 3.08$ $(\mathrm{m}, 1 \mathrm{H}), 4.15(\mathrm{dq}, J=11.1,6.3 \mathrm{~Hz}, 1 \mathrm{H}), 4.55(\mathrm{~d}, J=4.2 \mathrm{~Hz}, 1 \mathrm{H}), 7.10-7.30(\mathrm{~m}, 5 \mathrm{H}) ;{ }^{13} \mathrm{C}$ NMR 14.6, $51.2(\mathrm{q}, J=34.5 \mathrm{~Hz}), 56.9,76.3,113.6,121.8(\mathrm{q}, J=281.3 \mathrm{~Hz}), 126.4,127.9$, 128.3, 140.0; ${ }^{19}$ F NMR $\delta-75.0(\mathrm{~d}, J=6.3 \mathrm{~Hz}) . \mathrm{MS}(\mathrm{EI} 70 \mathrm{eV}) \mathrm{m} / z 259\left(\mathrm{M}^{+}+1\right), 241,232$, 214, 151 (100), 124, 108, 77. Anal. Calcd for $\mathrm{C}_{12} \mathrm{H}_{18} \mathrm{~F}_{3} \mathrm{~N}_{2} \mathrm{O}: \mathrm{C}, 55.81 ; \mathrm{H}, 5.07 ; \mathrm{N}, 10.85$. Found: C, 55.86; H, 5.27; N, 10.67. $\mathbf{b}_{\text {minor }}{ }^{1} \mathrm{H}$ NMR $\delta 0.89(\mathrm{~d}, J=6.8 \mathrm{~Hz}, 3 \mathrm{H}), 1.60(\mathrm{dd}, J=$ $11.8,3.8 \mathrm{~Hz}, 1 \mathrm{H}), 2.80(\mathrm{~s}, 1 \mathrm{H}), 3.08(\mathrm{~m}, 1 \mathrm{H}), 4.15(\mathrm{dq}, J=11.1,6.3 \mathrm{~Hz}, 1 \mathrm{H}), 4.51(\mathrm{~d}, J=3.7$ $\mathrm{Hz}, 1 \mathrm{H}), 7.10-7.30(\mathrm{~m}, 5 \mathrm{H}) ;{ }^{13} \mathrm{C}$ NMR $15.4,51.7$ (q, $\left.J=34.5 \mathrm{~Hz}\right), 56.9,75.5,114.2,121.5$ (q, $J=280.8 \mathrm{~Hz}), 126.5,127.8,128.3,139.9 ;{ }^{19} \mathrm{~F} \mathrm{NMR} \delta-74.8(\mathrm{~d}, J=6.3 \mathrm{~Hz})$.

\section{3,3,3-Trifluoro-2-((1S,2R)-2-hydroxy-1-methyl-2-phenylethylamino)-2-}

methylpropionitrile (6c). Following the same procedure than for the synthesis of $2 \mathbf{e}$ (see experimental section), the aminonitrile $\mathbf{6 c}$ was prepared from the oxazolidine $5 \mathbf{c}$ (245 $\mathrm{mg}, 1$ mmol). Purification by flash chromatography (85:15 petroleum ether/ethyl acetate) gave $\mathbf{6 c}$ $(218 \mathrm{mg}, 80 \%)$ as a $67: 33$ mixture of diastereomers. $\mathbf{6 c}_{\text {major }}$ IR (neat) $3358,2982,1604,1453$, $1178 \mathrm{~cm}^{-1} ;{ }^{1} \mathrm{H}$ NMR $\delta 0.93(\mathrm{~d}, J=6.5 \mathrm{~Hz}, 3 \mathrm{H}), 1.64(\mathrm{~s}, 3 \mathrm{H}), 2.40(\mathrm{~s}, 1 \mathrm{H}), 3.12(\mathrm{~m}, 1 \mathrm{H}), 4.82$ 
(m, 1H), 7.10-7.40 (m, 5H); ${ }^{13} \mathrm{C}$ NMR 15.4, 21.2, 56.0, $57.8(\mathrm{q}, J=30.6 \mathrm{~Hz}), 75.4,116.6$, $123.3(\mathrm{q}, J=284.7 \mathrm{~Hz}), 125.8,127.5,128.3,140.7 ;{ }^{19} \mathrm{~F}$ NMR $\delta-79.1(\mathrm{~s}) . \mathrm{MS}(\mathrm{EI} 70 \mathrm{eV}) \mathrm{m} / z$ $272\left(\mathrm{M}^{+}\right), 246,201,139(100), 124,77 . \mathbf{6 c}_{\text {minor }}{ }^{1} \mathrm{H}$ NMR $\delta 0.96(\mathrm{~d}, J=6.0 \mathrm{~Hz}, 3 \mathrm{H}), 1.58(\mathrm{~s}$, $3 \mathrm{H}), 3.20(\mathrm{~s}, 1 \mathrm{H}), 4.78(\mathrm{~m}, 1 \mathrm{H}), 7.10-7.40(\mathrm{~m}, 5 \mathrm{H}) ;{ }^{13} \mathrm{C} \mathrm{NMR} 15.2,20.3,55.2,57.8(\mathrm{q}, J=$ $30.6 \mathrm{~Hz}), 75.8,117.0,123.3(\mathrm{q}, J=284.7 \mathrm{~Hz}), 125.6,127.5,128.3,140.7 ;{ }^{19} \mathrm{~F} \mathrm{NMR} \delta-79.8$ $(\mathrm{s})$.

3,3,3-Trifluoro-2-((1R)-2-hydroxy-1-phenylethylamino)-propionitrile (8a). Following the same procedure than for the synthesis of $\mathbf{2 e}$ (see experimental section), the aminonitrile $\mathbf{8 a}$ was prepared from the oxazolidine $7 \mathbf{a}(3 \mathrm{~g}, 13.8 \mathrm{mmol})$. Purification by flash chromatography (85:15 petroleum ether/ethyl acetate) gave a pure isolated fraction of $(\boldsymbol{R}, \boldsymbol{R})-8 \mathbf{a}(1.7 \mathrm{~g}, 46 \%)$ as a pale yellow liquid and a fraction containing a 54:46 mixture of $(\boldsymbol{R}, \boldsymbol{R})-\mathbf{8 a}$ and $(\boldsymbol{S}, \boldsymbol{R})-\mathbf{8 a}(1.25$ g, 37\%). ( $\boldsymbol{R}, \boldsymbol{R})-8 \mathbf{a} . \mathrm{R}_{\mathrm{f}}=0.25(85: 15$ petroleum ether/ethyl acetate $) ;[\alpha]^{20}{ }_{\mathrm{D}}+203(c 0.76$, $\mathrm{CHCl}_{3}$ ); IR (neat) 3338, 3020, 2934, 1216, $1150 \mathrm{~cm}^{-1} ;{ }^{1} \mathrm{H} \mathrm{NMR} \delta 1.83(\mathrm{dd}, J=6.6,3.6 \mathrm{~Hz}$, $1 \mathrm{H}), 2.87(\mathrm{~d}, J=13.2 \mathrm{~Hz}, 1 \mathrm{H}), 3.65(\mathrm{dt}, J=9.9,6.6 \mathrm{~Hz}, 1 \mathrm{H}), 3.82(\mathrm{ddd}, J=9.9,9.8,3.6 \mathrm{~Hz}$, $1 \mathrm{H}), 3.90(\mathrm{dq}, J=13.2,6.7 \mathrm{~Hz}, 1 \mathrm{H}), 4.13(\mathrm{dd}, J=6.6,3.6 \mathrm{~Hz}, 1 \mathrm{H}), 7.20-7.40(\mathrm{~m}, 5 \mathrm{H}) ;{ }^{13} \mathrm{C}$ NMR $\delta 50.9(q, J=34.5 \mathrm{~Hz}), 62.5,66.9,113.4,121.6(\mathrm{q}, J=281.3 \mathrm{~Hz}), 126.8,127.5,129.2$, 136.3; ${ }^{19} \mathrm{~F}$ NMR $\delta-74.2(\mathrm{~d}, J=6.7 \mathrm{~Hz}) ; \mathrm{MS}(\mathrm{EI} 70 \mathrm{eV}) \mathrm{m} / z 245\left(\mathrm{M}^{+}+1\right), 227,218,213$ (100), 193, 180, 143, 135, 121. Anal. Calcd for $\mathrm{C}_{11} \mathrm{H}_{11} \mathrm{~F}_{3} \mathrm{~N}_{2} \mathrm{O}$ : C, 54.10; H, 4.54; N, 11.17. Found: C, 54.23; H, 4.52; N, 11.13. $(\boldsymbol{S}, \boldsymbol{R})-8 \mathbf{a} . \mathrm{Rf}=0.18$ (85:15 petroleum ether/ethyl acetate); ${ }^{1} \mathrm{H}$ NMR $\delta 2.05(\mathrm{~s}, 1 \mathrm{H}), 2.60(\mathrm{~d}, J=12.5 \mathrm{~Hz}, 1 \mathrm{H}), 3.72(\mathrm{dd}, J=10.8,8.4 \mathrm{~Hz}, 1 \mathrm{H}), 3.80(\mathrm{dd}, J$ $=10.8,3.8 \mathrm{~Hz}, 1 \mathrm{H}), 4.12(\mathrm{dd}, J=8.4,3.8 \mathrm{~Hz}, 1 \mathrm{H}), 4.35(\mathrm{dq}, J=12.5,6.4 \mathrm{~Hz}, 1 \mathrm{H}), 7.30-7.50$ $(\mathrm{m}, 5 \mathrm{H}) ;{ }^{13} \mathrm{C} \mathrm{NMR} \delta 51.0(\mathrm{q}, J=34.5 \mathrm{~Hz}), 62.9,67.2,122.2(\mathrm{q}, J=281.3 \mathrm{~Hz}), 127.5,128.3$, 129.3, 135.9, 138.6; ${ }^{19} \mathrm{~F} \mathrm{NMR} \delta-73.9(\mathrm{~d}, J=6.4 \mathrm{~Hz})$. 


\section{3,3,3-Trifluoro-2-((1R)-benzyl-2-hydroxy-1-phenylethylamino)-propionitrile (8b).}

Following the same procedure than for the synthesis of $\mathbf{2 e}$ (see experimental section), the aminonitrile $\mathbf{8 b}$ was prepared from the oxazolidine $\mathbf{7 b}$ (310 $\mathrm{mg}, 1 \mathrm{mmol}$ ). Purification by flash chromatography (85:15 petroleum ether/ethyl acetate) gave $\mathbf{8 b}(291 \mathrm{mg}, 87 \%)$ as a 50:50 mixture of diastereomers. A pure analytical sample of $\mathbf{8 b}_{\text {major }}$.was isolated as a yellow oil. 8b $\mathbf{b}_{\text {major }}[\alpha]^{20}{ }_{\mathrm{D}}+80\left(c 0.1, \mathrm{CHCl}_{3}\right)$; IR (neat) 3454, 3032, 2942, 1604, 1496, 1454, 1195 $\mathrm{cm}^{-1}$; ${ }^{1} \mathrm{H}$ NMR $3.92(\mathrm{t}, J=4.1 \mathrm{~Hz}, 1 \mathrm{H}), 4.12(\mathrm{~d}, J=14.1 \mathrm{~Hz}, 1 \mathrm{H}), 4.17(\mathrm{dd}, J=11.6,4.1 \mathrm{~Hz}$, $1 \mathrm{H}), 4.27(\mathrm{~d}, J=14.1 \mathrm{~Hz}, 1 \mathrm{H}), 4.34(\mathrm{dd}, J=11.6,4.1 \mathrm{~Hz}, 1 \mathrm{H}), 4.56(\mathrm{q}, J=7.0 \mathrm{~Hz}, 1 \mathrm{H}), 7.20$ $7.50(\mathrm{~m}, 10 \mathrm{H}) ;{ }^{13} \mathrm{C}$ NMR 53.8, $54.2(\mathrm{q}, J=34.4 \mathrm{~Hz}), 59.8,61.4,113.1,122.1(\mathrm{q}, J=283.7$ $\mathrm{Hz}), 128.0,128.1,128.5,128.7,128.8,128.8,137.1,137.2 ;{ }^{19} \mathrm{~F}$ NMR $\delta-72.5$ (d, $\left.J=7.0 \mathrm{~Hz}\right)$; MS (EI $70 \mathrm{eV}) \mathrm{m} / z 334\left(\mathrm{M}^{+}\right), 304,302,238,187,91$ (100). Anal. Calcd for $\mathrm{C}_{18} \mathrm{H}_{17} \mathrm{~F}_{3} \mathrm{~N}_{2} \mathrm{O}: \mathrm{C}$,

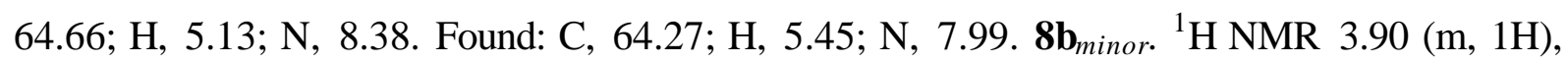
$4.10(\mathrm{~m}, 1 \mathrm{H}), 4.13(\mathrm{~d}, J=14.6 \mathrm{~Hz}, 1 \mathrm{H}), 4.33(\mathrm{~d}, J=14.6 \mathrm{~Hz}, 1 \mathrm{H}), 4.74(\mathrm{q}, J=7.0 \mathrm{~Hz}, 1 \mathrm{H})$, $7.20-7.50(\mathrm{~m}, 10 \mathrm{H}) ;{ }^{13} \mathrm{C} \mathrm{NMR}, 53.3(\mathrm{q}, J=34.0 \mathrm{~Hz}), 54.9,62.3,63.8,111.9,122.4$ (q, $J=$ $282.7 \mathrm{~Hz}), 127.8,127.9,128.3,128.4,129.0,129.1,134.3,136.8 ;{ }^{19} \mathrm{~F}$ NMR $\delta-72.3(\mathrm{~d}, J=7.0$ $\mathrm{Hz})$.

\section{3,3,3-Trifluoro-2-((1R)-2-hydroxy-1-phenylethylamino)-2-phenylpropionitrile (8d).}

Following the same procedure than for the synthesis of $\mathbf{2 e}$ (see experimental section), the aminonitrile 8d was prepared from the oxazolidine $7 \mathbf{d}(1.9 \mathrm{~g}, 6.4 \mathrm{mmol})$. Purification by flash chromatography (85:15 petroleum ether/ethyl acetate) gave pure isolated $(\boldsymbol{R}, \boldsymbol{R})-8 \mathbf{d}(1.43 \mathrm{~g}$, $70 \%)$ and $(\boldsymbol{S}, \boldsymbol{R})-8 d(556 \mathrm{mg}, 27 \%)$. (R, R)-8d. Yellow oil; $[\alpha]^{20}{ }_{\mathrm{D}}-101.5\left(c \quad 0.9, \mathrm{CHCl}_{3}\right) ;{ }^{1} \mathrm{H}$ NMR $\delta 2.00(\mathrm{~s}, 1 \mathrm{H}), 2.84(\mathrm{~s}, 1 \mathrm{H}), 3.43(\mathrm{dd}, J=11.3,8.0 \mathrm{~Hz}, 1 \mathrm{H}), 3.54(\mathrm{dd}, J=11.3,4.7 \mathrm{~Hz}$, $1 \mathrm{H}), 3.54(\mathrm{dd}, J=8.0,4.7 \mathrm{~Hz}, 1 \mathrm{H}), 7.00-7.30(\mathrm{~m}, 5 \mathrm{H}), 7.40(\mathrm{~m}, 3 \mathrm{H}), 7.70(\mathrm{~m}, 2 \mathrm{H}) ;{ }^{13} \mathrm{C} \mathrm{NMR}$ $\delta 59.7,65.8,65.9(\mathrm{q}, J=30.3 \mathrm{~Hz}), 114.1,121.7(\mathrm{q}, J=285.3 \mathrm{~Hz}), 126.6,127.3,127.5,127.8$, 
129.1, 129.7, 129.7, 137.9; ${ }^{19}$ F NMR $\delta-76.1$ (s). $(\boldsymbol{S}, \boldsymbol{R})$-8d. Yellow oil; $[\alpha]^{20}{ }_{\mathrm{D}}-121.7(c 0.6$,

$\mathrm{CHCl}_{3}$ ); IR (neat) 3333, 3031, 2925, 1603, 1492, 1453, $1174 \mathrm{~cm}^{-1} ;{ }^{1} \mathrm{H}$ NMR $\delta 1.96$ (s, 1H), $3.17(\mathrm{~s}, 1 \mathrm{H}), 3.61(\mathrm{dd}, J=13.0,8.8 \mathrm{~Hz}, 1 \mathrm{H}), 3.83(\mathrm{dd}, J=8.8,4.2 \mathrm{~Hz}, 1 \mathrm{H}), 3.86(\mathrm{dd}, J=13.0$, $4.2 \mathrm{~Hz}, 1 \mathrm{H}), 6.90-7.20(\mathrm{~m}, 8 \mathrm{H}), 7.40(\mathrm{~m}, 2 \mathrm{H}) ;{ }^{13} \mathrm{C} \mathrm{NMR} \delta 60.9,65.9,68.4(\mathrm{q}, J=29.9 \mathrm{~Hz})$, $116.1,122.5(\mathrm{q}, J=285.4 \mathrm{~Hz}), 127.3,127.5,128.2,128.3,128.5,129.0,130.3,139.7 ;{ }^{19} \mathrm{~F}$ NMR $\delta$-76.6 (s); MS (EI $70 \mathrm{eV}) \mathrm{m} / z 320\left(\mathrm{M}^{+}\right), 289,262,224,192,105,91$ (100), 77. Anal. Calcd for $\mathrm{C}_{17} \mathrm{H}_{15} \mathrm{~F}_{3} \mathrm{~N}_{2} \mathrm{O}: \mathrm{C}, 63.75 ; \mathrm{H}, 4.72 ; \mathrm{N}, 8.75$. Found: C, 63.51; H, 4.79; N, 8.74.

\section{$(R)$ - and (S)-Trifluoromethyl alanine synthesis.}

Synthesis of $(R)$ - and $(S)$-trifluoromethylalanine. Methyl (2R)-3,3,3-trifluoro-2-((1R)-2hydroxy-1-phenylethylamino)-2-methylpropionate $((\boldsymbol{R}, \boldsymbol{R})-9 \mathrm{e})$. To a solution of amino nitrile $(\boldsymbol{R}, \boldsymbol{R})$-2e $(370 \mathrm{mg}, 1.4 \mathrm{mmol})$ in dry methanol $(5 \mathrm{~mL})$ at $0{ }^{\circ} \mathrm{C}$ was added a saturated solution of dry $\mathrm{HCl}$ in methanol $(10 \mathrm{~mL})$. The mixture was refluxed for $6 \mathrm{~h}$ and concentrated under reduced pressure. The crude mixture was treated with a saturated aqueous solution of $\mathrm{NaHCO}_{3}(50 \mathrm{~mL})$, and the aqueous layer was extracted with diethyl ether $(3 \times 10 \mathrm{~mL})$. The combined organic extracts were washed with brine $(10 \mathrm{~mL})$, dried over $\mathrm{Na}_{2} \mathrm{SO}_{4}$, filtered, and concentrated under reduced pressure. Purification by flash chromatography (4:1 petroleum ether/ethyl acetate) gave $(\boldsymbol{R}, \boldsymbol{R})-9 \mathrm{e}(325 \mathrm{mg}, 80 \%)$ as a yellow oil. $[\alpha]^{20}{ }_{\mathrm{D}}-113.4(c \quad 0.7$, $\mathrm{CHCl}_{3}$ ); IR (neat) 3334, 3029, 2957, 1740, 1436, $1165 \mathrm{~cm}^{-1} ;{ }^{1} \mathrm{H}$ NMR $\delta 1.19(\mathrm{~s}, 3 \mathrm{H}), 3.38$ $(\mathrm{dd}, J=11.0,9.1 \mathrm{~Hz}, 1 \mathrm{H}), 3.58(\mathrm{dd}, J=11.0,4.4 \mathrm{~Hz}, 1 \mathrm{H}), 3.64(\mathrm{~s}, 3 \mathrm{H}), 3.77(\mathrm{dd}, J=9.1,4.4$ $\mathrm{Hz}, 1 \mathrm{H}), 7.10-7.30(\mathrm{~m}, 5 \mathrm{H}) ;{ }^{13} \mathrm{C} \mathrm{NMR} \delta 17.4,53.1,60.2,65.8(\mathrm{q}, J=27.1 \mathrm{~Hz}), 67.7,124.6$ $(\mathrm{q}, J=284.9 \mathrm{~Hz}), 126.7,127.5,129.5,141.6 ;{ }^{19} \mathrm{~F} \mathrm{NMR} \delta-77.7(\mathrm{~s}) ; \mathrm{MS}(\mathrm{CI}) \mathrm{m} / \mathrm{z} 318(\mathrm{M}+$ $\mathrm{NH}_{3}$ ), 277, 217 (100), 189, 137. Anal. Calcd for $\mathrm{C}_{13} \mathrm{H}_{16} \mathrm{~F}_{3} \mathrm{NO}_{3}: \mathrm{C}, 53.61 ; \mathrm{H}, 5.54 ; \mathrm{N}, 4.81$. Found: C, 53.24; H, 5.37; N, 4.78. 
(R)-Methyl trifluoromethylalaninate hydrochloride $((\boldsymbol{R})$-10e). To a solution of aminoester $(R, R)$-9e $(230 \mathrm{mg}, 0.8 \mathrm{mmol})$ in methanol $(7 \mathrm{~mL})$ was added $\mathrm{Pd}(\mathrm{OH})_{2}(0.16 \mathrm{mmol})$. The mixture was stirred under $\mathrm{H}_{2}$ atmosphere until complete conversion of 9e. The catalyst was removed by filtration and $1 \mathrm{~N} \mathrm{HCl}(10 \mathrm{~mL})$ and ether $(10 \mathrm{~mL})$ were added. The aqueous layer was concentrated under reduced pressure and $(\boldsymbol{R})$-10e was obtained in a quantitative yield as a pale yellow solid. Mp $84-86{ }^{\circ} \mathrm{C} ;[\alpha]^{20}{ }_{\mathrm{D}}+2.6\left(c 0.80, \mathrm{H}_{2} \mathrm{O}\right)\left([\alpha]^{20}{ }_{\text {Dlitt }}+2.6\left(c 0.72, \mathrm{H}_{2} \mathrm{O}\right) ;{ }^{5}{ }^{1} \mathrm{H}\right.$ NMR $\delta\left(\mathrm{D}_{2} \mathrm{O}\right) 1.80(\mathrm{~s}, 3 \mathrm{H}), 3.93(\mathrm{~s}, 3 \mathrm{H}),{ }^{13} \mathrm{C} \mathrm{NMR} \delta\left(\mathrm{D}_{2} \mathrm{O}\right)$ 18.9, 57.6, 64.2 (q, $\left.J=31.2 \mathrm{~Hz}\right)$ $124.9(\mathrm{q}, J=283.1 \mathrm{~Hz}), 168.0 ;{ }^{19} \mathrm{~F}$ NMR $\delta\left(\mathrm{D}_{2} \mathrm{O}\right)-74.9(\mathrm{~s})$.

$(\boldsymbol{R})$-Trifluoromethylalanine hydrochloride $((\boldsymbol{R})-\mathbf{1 1 e})$. To a solution $6 \mathrm{~N} \mathrm{HCl} / \mathrm{AcOH}(6: 1)$ $(10 \mathrm{~mL})$ was added $(\boldsymbol{R})-\mathbf{1 0 e}$. The mixture was warmed to reflux for $8 \mathrm{~h}$. The mixture was concentrated under reduced pressure and $(\boldsymbol{R})-\mathbf{1 1 e}$ was obtained in a quantitative yield as a yellow solid. $\mathrm{Mp}>220{ }^{\circ} \mathrm{C} ;[\alpha]^{20}{ }_{\mathrm{D}}+10.9\left(c\right.$ 0.90, $\left.\mathrm{H}_{2} \mathrm{O}\right)\left([\alpha]^{20}{ }_{\text {Dlitt }}+11.4\left(c 1.04, \mathrm{H}_{2} \mathrm{O}\right) ;{ }^{5}{ }^{1} \mathrm{H}\right.$ $\operatorname{NMR} \delta\left(\mathrm{D}_{2} \mathrm{O}\right) 1.76(\mathrm{~s}, 3 \mathrm{H}) ;{ }^{13} \mathrm{C} \mathrm{NMR} \delta\left(\mathrm{D}_{2} \mathrm{O}\right) 19.2,64.7(\mathrm{q}, J=28.0 \mathrm{~Hz}), 125.9(\mathrm{q}, J=$ $281.0 \mathrm{~Hz}), 170.3 ;{ }^{19} \mathrm{~F} \mathrm{NMR} \delta\left(\mathrm{D}_{2} \mathrm{O}\right)-74.7(\mathrm{~s})$.

$(\boldsymbol{R})$-Trifluoromethylalanine $((\boldsymbol{R})-\mathbf{1 2 e})$. A solution of $(\boldsymbol{R})-11 \mathrm{e}(116 \mathrm{mg}, 1 \mathrm{mmol})$ in propene oxide $(5 \mathrm{~mL})$ was stirred for $24 \mathrm{~h}$ at room temperature. The solvent was removed under reduced pressure and the crude mixture was purified by recrystallization in $\mathrm{MeOH}$ to give pure $(R)$-Trifluoromethylalanine $(\boldsymbol{R})-\mathbf{1 2 e}(99 \mathrm{mg}, 82 \%)$ as a white solid. ${ }^{5}$ Mp (sublimate); $[\alpha]^{20}{ }_{\mathrm{D}}+11.2(c 0.90,1 \mathrm{~N} \mathrm{HCl}) ;{ }^{1} \mathrm{H}$ NMR $\delta\left(\mathrm{D}_{2} \mathrm{O}\right) 1.55(\mathrm{~s}, 3 \mathrm{H}) ;{ }^{13} \mathrm{C} \mathrm{NMR} \delta\left(\mathrm{D}_{2} \mathrm{O}\right) 20.9,64.1$ $(\mathrm{q}, J=27.0 \mathrm{~Hz}), 127.5(\mathrm{q}, J=281.0 \mathrm{~Hz}), 174.7 ;{ }^{19} \mathrm{~F} \mathrm{NMR} \delta\left(\mathrm{D}_{2} \mathrm{O}\right)-75.8(\mathrm{~s})$.

(5) Bravo, P. ; Capelli, S. ; Meille, S.V. ; Viani, F.; Zanda, M. ; Kukhar, V.P.; Soloshonok, V.

A. Tetrahedron : Asymmetry. 1994, 5, 2009-2018 
(S)-Trifluoromethylalanine hydrochloride $((S)-11 \mathrm{e})$. A solution of aminonitrile $(S, R)-2 \mathrm{e}$ (260 $\mathrm{mg}, 1 \mathrm{mmol})$ in conc. $\mathrm{HCl}(5 \mathrm{~mL})$ was warmed to reflux for $14 \mathrm{~h}$. The mixture was cooled down to room temperature and the aqueous layer was extracted with diethyl ether $(5 \mathrm{~mL})$. The aqueous layer was concentrated under reduced pressure to afford $(\boldsymbol{S})$-11e (116 mg, 60\%) as a yellow solid. $\mathrm{Mp}>220{ }^{\circ} \mathrm{C} ;[\alpha]^{20}{ }_{\mathrm{D}}-10.4\left(c \quad 0.50, \mathrm{H}_{2} \mathrm{O}\right)\left([\alpha]^{20}{ }_{\text {Dlitt }}-10.5(c\right.$ $\left.0.58, \mathrm{H}_{2} \mathrm{O}\right) ;{ }^{5} \mathrm{H},{ }^{13} \mathrm{C},{ }^{19} \mathrm{~F}$ NMR data identical to those of $(\boldsymbol{R})-\mathbf{1 1 e}$.

\section{Synthesis of diamines and amino alcohols.}

(2R)-3,3,3-Trifluoro-2-((1R)-2-hydroxy-1-phenylethylamino)-2-phenylpropylamine

$((\boldsymbol{R}, \boldsymbol{R})-\mathbf{1 3 d}) . \quad(\boldsymbol{R}, \boldsymbol{R})-\mathbf{1 3 d}$ was synthesized according to the same procedure as $(\boldsymbol{R}, \boldsymbol{R})-\mathbf{1 3 a}$, starting from $(\boldsymbol{R}, \boldsymbol{R})-\mathbf{8 d}(320 \mathrm{mg}, 1 \mathrm{mmol}) .(\boldsymbol{R}, \boldsymbol{R})-\mathbf{1 3 d}(233 \mathrm{mg}, 72 \%)$ was obtained as a yellow oil. $[\alpha]^{20}{ }_{\mathrm{D}}-11.5\left(c\right.$ 0.9, $\left.\mathrm{CHCl}_{3}\right)$; IR (neat) $3363,3023,2927,1454,1148,702 \mathrm{~cm}^{-1}$; ${ }^{1} \mathrm{H} \mathrm{NMR}$ $\delta 2.30(\mathrm{br}, 4 \mathrm{H}), 3.32(\mathrm{~d}, J=14.0 \mathrm{~Hz}, 1 \mathrm{H}), 3.45(\mathrm{~d}, J=14.0 \mathrm{~Hz}, 1 \mathrm{H}), 3.49(\mathrm{dd}, J=11.0,9.0$ $\mathrm{Hz}, 1 \mathrm{H}), 3.62(\mathrm{dd}, J=11.0,3.5 \mathrm{~Hz}, 1 \mathrm{H}), 4.05(\mathrm{dd}, J=9.0,3.5 \mathrm{~Hz}, 1 \mathrm{H}), 7.10-7.50(\mathrm{~m}, 10 \mathrm{H})$; ${ }^{13} \mathrm{C}$ NMR $\delta$ 40.7, 58.7, $66.1(\mathrm{q}, J=23.5 \mathrm{~Hz}), 67.9,126.1(\mathrm{q}, J=287.8 \mathrm{~Hz}), 126.8,126.9$, 127.8, 128.0, 128.4, 128.5, 135.6, 142.4; ${ }^{19} \mathrm{~F}$ NMR $\delta-73.5(\mathrm{~s})$; MS (EI $\left.70 \mathrm{eV}\right) \mathrm{m} / \mathrm{z} 324\left(\mathrm{M}^{+}\right)$ 304, 293 (100), 274, 243, 173, 121, 77.

\section{(2S)-3,3,3-Trifluoro-2-((1R)-2-hydroxy-1-phenylethylamino)-2-phenylpropylamine}

$((\boldsymbol{S}, \boldsymbol{R})-\mathbf{1 3 d}) .(\boldsymbol{S}, \boldsymbol{R})-\mathbf{1 3 d}$ was synthesized according to the same procedure as $(\boldsymbol{R}, \boldsymbol{R})-\mathbf{1 3 a}$, starting from $(\boldsymbol{S}, \boldsymbol{R})-\mathbf{8 d}(400 \mathrm{mg}, 1.25 \mathrm{mmol}) .(\boldsymbol{S}, \boldsymbol{R})-\mathbf{1 3 d}(304 \mathrm{mg}, 75 \%)$ was obtained as a yellow oil. $[\alpha]^{20}{ }_{\mathrm{D}}-64.2\left(c\right.$ 2.0, $\left.\mathrm{CHCl}_{3}\right) ;{ }^{1} \mathrm{H} \mathrm{NMR} \delta 2.30(\mathrm{br}, 4 \mathrm{H}), 3.22(\mathrm{~d}, J=14.0 \mathrm{~Hz}, 1 \mathrm{H})$, $3.48(\mathrm{~d}, J=14.0 \mathrm{~Hz}, 1 \mathrm{H}), 3.49(\mathrm{dd}, J=9.7,7.0 \mathrm{~Hz}, 1 \mathrm{H}), 3.52(\mathrm{dd}, J=9.7,3.5 \mathrm{~Hz}, 1 \mathrm{H}), 3.72$ $(\mathrm{dd}, J=7.0,3.5 \mathrm{~Hz}, 1 \mathrm{H}), 7.00-7.40(\mathrm{~m}, 10 \mathrm{H}) ;{ }^{13} \mathrm{C} \mathrm{NMR} \delta 43.5,58.9,66.3(\mathrm{q}, J=22.5 \mathrm{~Hz})$, 
$67.7,126.7(\mathrm{q}, J=289.0 \mathrm{~Hz}), 126.8,127.0,127.9,128.0,128.1,128.2,135.4,142.8 ;{ }^{19} \mathrm{~F} \mathrm{NMR}$ $\delta-72.7(\mathrm{~s})$.

(2R)-3,3,3-Trifluoro-2-((1R)-2-hydroxy-1-phenylethylamino)-2-methylpropylamine $((\boldsymbol{R}, \boldsymbol{R})-\mathbf{1 3 e}) . \quad(\boldsymbol{R}, \boldsymbol{R})-\mathbf{1 3 e}$ was synthesized according to the same procedure as $(\boldsymbol{R}, \boldsymbol{R})-\mathbf{1 3 a}$, starting from $(\boldsymbol{R}, \boldsymbol{R})$-2e $(260 \mathrm{mg}, 1 \mathrm{mmol})$. After purification by recrystallization in pentane, $(\boldsymbol{R}, \boldsymbol{R})-13 \mathrm{e}(240 \mathrm{mg}, 92 \%)$ was obtained as a white solid. Mp $61-62{ }^{\circ} \mathrm{C} ;[\alpha]^{20} \mathrm{D}-81.7(c 0.8$, $\mathrm{CHCl}_{3}$ ); IR (KBr) 3376, 3303, 3029, 2885, 1461, $11603344 \mathrm{~cm}^{-1} ;{ }^{1} \mathrm{H}$ NMR $\delta 0.98$ (s, 3H); $2.68(\mathrm{~d}, J=14.1 \mathrm{~Hz}, 1 \mathrm{H}), 2.83(\mathrm{~d}, J=14.1 \mathrm{~Hz}, 1 \mathrm{H}), 3.35(\mathrm{dd}, J=10.5,9.0 \mathrm{~Hz}, 1 \mathrm{H}), 3.56(\mathrm{dd}$, $J=10.5,3.5 \mathrm{~Hz}, 1 \mathrm{H}), 3.92(\mathrm{dd}, J=9.0,3.5 \mathrm{~Hz}, 1 \mathrm{H}), 7.10-7.50(\mathrm{~m}, 5 \mathrm{H}) ;{ }^{13} \mathrm{C} \mathrm{NMR} \delta 17.8$ 43.6, 58.2, $60.2(\mathrm{q}, J=23.4 \mathrm{~Hz}), 67.5,126.7,127.1,127.4(\mathrm{q}, J=286.8 \mathrm{~Hz}), 129.7,142.6 ;{ }^{19} \mathrm{~F}$ NMR $\delta$-77.4 (s); MS (EI $70 \mathrm{eV}) \mathrm{m} / z 263\left(\mathrm{M}^{+}+1\right), 231$ (100), 200, 153, 121, 112, 103, 77. Anal. Calcd for $\mathrm{C}_{12} \mathrm{H}_{17} \mathrm{~F}_{3} \mathrm{~N}_{2} \mathrm{O}: \mathrm{C}, 54.95 ; \mathrm{H}, 6.53 ; \mathrm{N}, 10.68$. Found: C, 54.66; H, 6.64; N, 10.5.

\section{(2S)-3,3,3-Trifluoro-2-((1R)-2-hydroxy-1-phenylethylamino)-2-methylpropylamine}

$((\boldsymbol{S}, \boldsymbol{R})-\mathbf{1 3 e}) .(\boldsymbol{S}, \boldsymbol{R})$-13e was synthesized according to the same procedure as $(\boldsymbol{R}, \boldsymbol{R})-\mathbf{1 3 a}$, starting from $(S, \boldsymbol{R})-2 \mathrm{e}(260 \mathrm{mg}, 1 \mathrm{mmol})$. After purification by recrystallization in pentane, $(S, R)-13 \mathrm{e}(260 \mathrm{mg}, 98 \%)$ was obtained as a white solid. Mp $64-66{ }^{\circ} \mathrm{C} ;[\alpha]^{20} \mathrm{D}-25.9(c 0.3$, $\left.\mathrm{CHCl}_{3}\right) ;{ }^{1} \mathrm{H}$ NMR $\delta 1.08(\mathrm{~s}, 3 \mathrm{H}) ; 2.60(\mathrm{~d}, J=13.3 \mathrm{~Hz}, 1 \mathrm{H}), 2.95(\mathrm{~d}, J=13.3 \mathrm{~Hz}, 1 \mathrm{H}), 3.39$ $(\mathrm{dd}, J=10.8,9.4 \mathrm{~Hz}, 1 \mathrm{H}), 3.61(\mathrm{dd}, J=10.8,4.3 \mathrm{~Hz}, 1 \mathrm{H}), 4.07(\mathrm{dd}, J=9.4,4.3 \mathrm{~Hz}, 1 \mathrm{H})$, 7.10-7.40 (m, 5H); ${ }^{13} \mathrm{C}$ NMR $\delta 17.4,44.9,59.1,59.9(q, J=23.6 \mathrm{~Hz}), 67.9,126.6,127.3$, $127.7(\mathrm{q}, J=288.0 \mathrm{~Hz}), 129.5,142.6 ;{ }^{19} \mathrm{~F}$ NMR $\delta-77.8(\mathrm{~s})$. 
added sodium nitrite (138 mg, $2 \mathrm{mmol}$ ). The mixture was stirred for $2 \mathrm{~h}$ at reflux temperature and overnight at room temperature. The aqueous layer was extracted with diethyl ether $(3 \mathrm{x}$ $20 \mathrm{~mL}$ ). The organic layer was dried over $\mathrm{Na}_{2} \mathrm{SO}_{4}$ and concentrated under reduced pressure. The crude product was purified by silica gel chromatography (3:1 petroleum ether/ethyl acetate) to afford $(\boldsymbol{R}, \boldsymbol{R})-\mathbf{1 4 e}(214 \mathrm{mg}, 82 \%)$ as a white solid. $\mathrm{Mp} 87-88{ }^{\circ} \mathrm{C} ;[\alpha]^{20}{ }_{\mathrm{D}}-41.2(c$ 0.8, $\mathrm{CHCl}_{3}$ ); IR (KBr) 3607, 3360, 3018, 2924, 1445, $1150 \mathrm{~cm}^{-1} ;{ }^{1} \mathrm{H}$ NMR $\delta 1.19$ (s, 3H), $3.38(\mathrm{dd}, J=10.9,8.6 \mathrm{~Hz}, 1 \mathrm{H}), 3.52(\mathrm{~s}, 2 \mathrm{H}), 3.56(\mathrm{dd}, J=10.9,4.6 \mathrm{~Hz}, 1 \mathrm{H}), 3.96(\mathrm{dd}, J=8.6$, $4.6 \mathrm{~Hz}, 1 \mathrm{H}), 7.10-7.40(\mathrm{~m}, 5 \mathrm{H}) ;{ }^{13} \mathrm{C} \mathrm{NMR} \delta 18.3,47.2,58.6,60.5(\mathrm{q}, J=25.3 \mathrm{~Hz}), 67.7$, $126.4(\mathrm{q}, J=288.0 \mathrm{~Hz}), 126.8,127.6,128.5,141.5 ;{ }^{19} \mathrm{~F}$ NMR $\delta-76.7(\mathrm{~s}) ; \mathrm{MS}(\mathrm{EI} 70 \mathrm{eV}) \mathrm{m} / \mathrm{z}$ $263\left(\mathrm{M}^{+}\right), 248,214(100), 154,103,77$. Anal. Calcd for $\mathrm{C}_{12} \mathrm{H}_{16} \mathrm{~F}_{3} \mathrm{NO}_{2}: \mathrm{C}, 54.75 ; \mathrm{H}, 6.13 ; \mathrm{N}$, 5.32. Found: C, 54.74; H, 5.90; N, 5.21.

(2S)-3,3,3-Trifluoro-2-((1R)-2-hydroxy-1-phenylethylamino)-2-methylpropanol

$((S, R)-$

14e). $(\boldsymbol{S}, \boldsymbol{R})$-14e was synthesized according to the same procedure as $(\boldsymbol{R}, \boldsymbol{R})-\mathbf{1 4} \mathbf{e}$, starting from $(\boldsymbol{S}, \boldsymbol{R})$-13e (260 mg, $1 \mathrm{mmol})$. After purification by silica gel chromatography (3:1 petroleum ether/ethyl acetate), $(S, R)-\mathbf{1 4 e}(193 \mathrm{mg}, 74 \%)$ was obtained as a colorless oil. $[\alpha]^{20}{ }_{\mathrm{D}}-7.7(c$ 1.0, $\left.\mathrm{CHCl}_{3}\right) ;{ }^{1} \mathrm{H}$ NMR $\left.\delta 1.09(\mathrm{~s}, 3 \mathrm{H}), 3.31(\mathrm{~d}, J=11.8 \mathrm{~Hz}, 1 \mathrm{H}),\right), 3.57(\mathrm{~d}, J=11.8 \mathrm{~Hz}, 1 \mathrm{H})$, $3.36(\mathrm{dd}, J=11.0,9.0 \mathrm{~Hz}, 1 \mathrm{H}), 3.51(\mathrm{dd}, J=11.0,4.5 \mathrm{~Hz}, 1 \mathrm{H}), 3.94(\mathrm{dd}, J=9.0,4.5 \mathrm{~Hz}, 1 \mathrm{H})$, 7.00-7.40 (m, 5H); ${ }^{13} \mathrm{C}$ NMR $\delta$ 17.6, 47.3, 59.4, $60.6(\mathrm{q}, J=25.3 \mathrm{~Hz}), 67.1,126.8,126.9(\mathrm{q}$, $J=287.8 \mathrm{~Hz}), 127.8,128.6,140.9 ;{ }^{19} \mathrm{~F}$ NMR $\delta-76.7(\mathrm{~s})$.

Removal of the $(R)$-phenylglycinol side chain, representative procedure. $(2 R)-3,3,3-$ Trifluoro-1,2-propanediamine hydrochloride $((\boldsymbol{R})-\mathbf{1 5 a})$. To a solution of $(\boldsymbol{R}, \boldsymbol{R})-\mathbf{1 3 a}$ (198 $\mathrm{mg}, 0.8 \mathrm{mmol})$ in a 2:1 mixture of $\mathrm{MeOH} / \mathrm{CH}_{2} \mathrm{Cl}_{2}(10 \mathrm{~mL})$ was added at $0{ }^{\circ} \mathrm{C} \mathrm{Pb}(\mathrm{OAc})_{4}(480$ $\mathrm{mg}, 1.2 \mathrm{mmol}$ ). After $20 \mathrm{~min}$ of stirring at $0^{\circ} \mathrm{C}$, the reaction mixture was poured in a buffer 
aqueous solution $\mathrm{pH}=7(10 \mathrm{~mL})$ at room temperature, then filtered on Celite. The aqueous layer was extracted with dichloromethane $(4 \times 10 \mathrm{~mL})$ and the combined organic extracts were dried over $\mathrm{Na}_{2} \mathrm{SO}_{4}$, filtered, and concentrated under reduced pressure. The mixture was then treated with $3 \mathrm{~N} \mathrm{HCl}(5 \mathrm{~mL})$ and diethyl ether $(5 \mathrm{~mL})$. The aqueous layer was concentrated under reduced pressure to afford $(\boldsymbol{R})-\mathbf{1 5 a}(93 \mathrm{mg}, 71 \%)$ as a yellow solid. $[\alpha]^{20}{ }_{\mathrm{D}}+1.2(c 1.1$, $1 \mathrm{~N} \mathrm{HCl})$; IR (KBr) 3483, 2952, 1581, 1216, $1184 \mathrm{~cm}^{-1} ;{ }^{1} \mathrm{H}$ NMR $\delta\left(\mathrm{D}_{2} \mathrm{O}\right) 3.81(\mathrm{dd}, J=14.6$, $5.9 \mathrm{~Hz}, 1 \mathrm{H}), 3.94(\mathrm{dd}, J=14.6,6.5 \mathrm{~Hz}, 1 \mathrm{H}), 4.86(\mathrm{dqd}, J=6.5,6.3,5.9 \mathrm{~Hz}, 1 \mathrm{H}) ;{ }^{13} \mathrm{C} \mathrm{NMR} \delta$ $\left(\mathrm{D}_{2} \mathrm{O}\right) 36.8,50.7(\mathrm{q}, J=32.8 \mathrm{~Hz}), 123.3(\mathrm{q}, J=281.0 \mathrm{~Hz}) ;{ }^{19} \mathrm{~F}$ NMR $\delta\left(\mathrm{D}_{2} \mathrm{O}\right)-72.8(\mathrm{~d}, J=6.3$ $\mathrm{Hz}) ; \mathrm{MS}(\mathrm{EI} 70 \mathrm{eV}) \mathrm{m} / z, 129\left(\mathrm{M}^{+}-\mathrm{Cl}\right)$.

(2R)-3,3,3-Trifluoro-2-methyl-1,2-propanediamine hydrochloride $((R)-15 \mathrm{e}) .(R)-15 \mathrm{e}$ was synthesized according to the same procedure as $(\boldsymbol{R})$-15a, starting from $(\boldsymbol{R}, \boldsymbol{R})-\mathbf{1 3 e}(240 \mathrm{mg}$, $0.92 \mathrm{mmol})$. (R)-15e (145 mg, 75\%) was obtained as a white solid. $[\alpha]^{20}{ }_{\mathrm{D}}-3.1(c 0.5,1 \mathrm{~N}$ $\mathrm{HCl})$; IR (KBr) 3425, 2924, 1621, $1192 \mathrm{~cm}^{-1} ;{ }^{1} \mathrm{H}$ NMR $\delta\left(\mathrm{D}_{2} \mathrm{O}\right) 1.87(\mathrm{~s}, 3 \mathrm{H}), 3.72(\mathrm{~d}, J=$ 14.6 Hz, 1H), $3.84(\mathrm{~d}, J=14.6 \mathrm{~Hz}, 1 \mathrm{H}) ;{ }^{13} \mathrm{C} \mathrm{NMR} \delta\left(\mathrm{D}_{2} \mathrm{O}\right) 16.6,41.7,57.5(\mathrm{q}, J=30.1 \mathrm{~Hz})$, $122.6(\mathrm{q}, J=284.2 \mathrm{~Hz}) ;{ }^{19} \mathrm{~F}$ NMR $\delta\left(\mathrm{D}_{2} \mathrm{O}\right)-77.9(\mathrm{~s}) ; \mathrm{MS}(\mathrm{EI} 70 \mathrm{eV}) \mathrm{m} / \mathrm{z} 142\left(\mathrm{M}^{+}-\mathrm{Cl}\right)$.

(2S)-3,3,3-Trifluoro-2-methyl-1,2-propanediamine hydrochloride $((S)-15 \mathrm{e}) . \quad(S)$-15e was synthesized according to the same procedure as $(\boldsymbol{R})$-15a, starting from $(\boldsymbol{S}, \boldsymbol{R})$-13e $(260 \mathrm{mg}$, $0.98 \mathrm{mmol})$. (S)-15e $(123 \mathrm{mg}, 60 \%)$ was obtained as a white solid. $[\alpha]^{20}{ }_{\mathrm{D}}+3.2(c 1.0,1 \mathrm{~N}$ $\mathrm{HCl})$; spectral data identical to $(\boldsymbol{R})-\mathbf{1 5 e}$. 


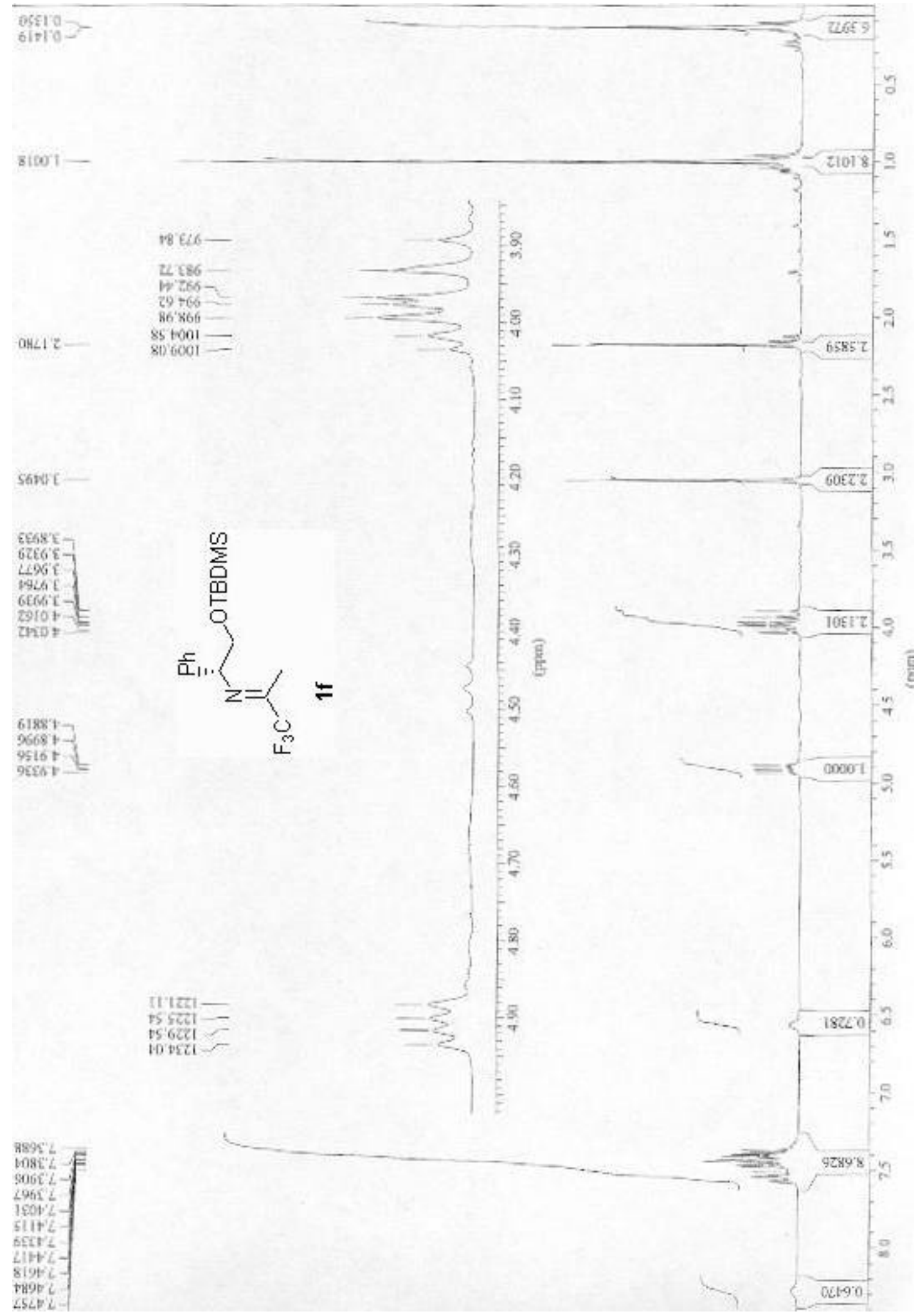




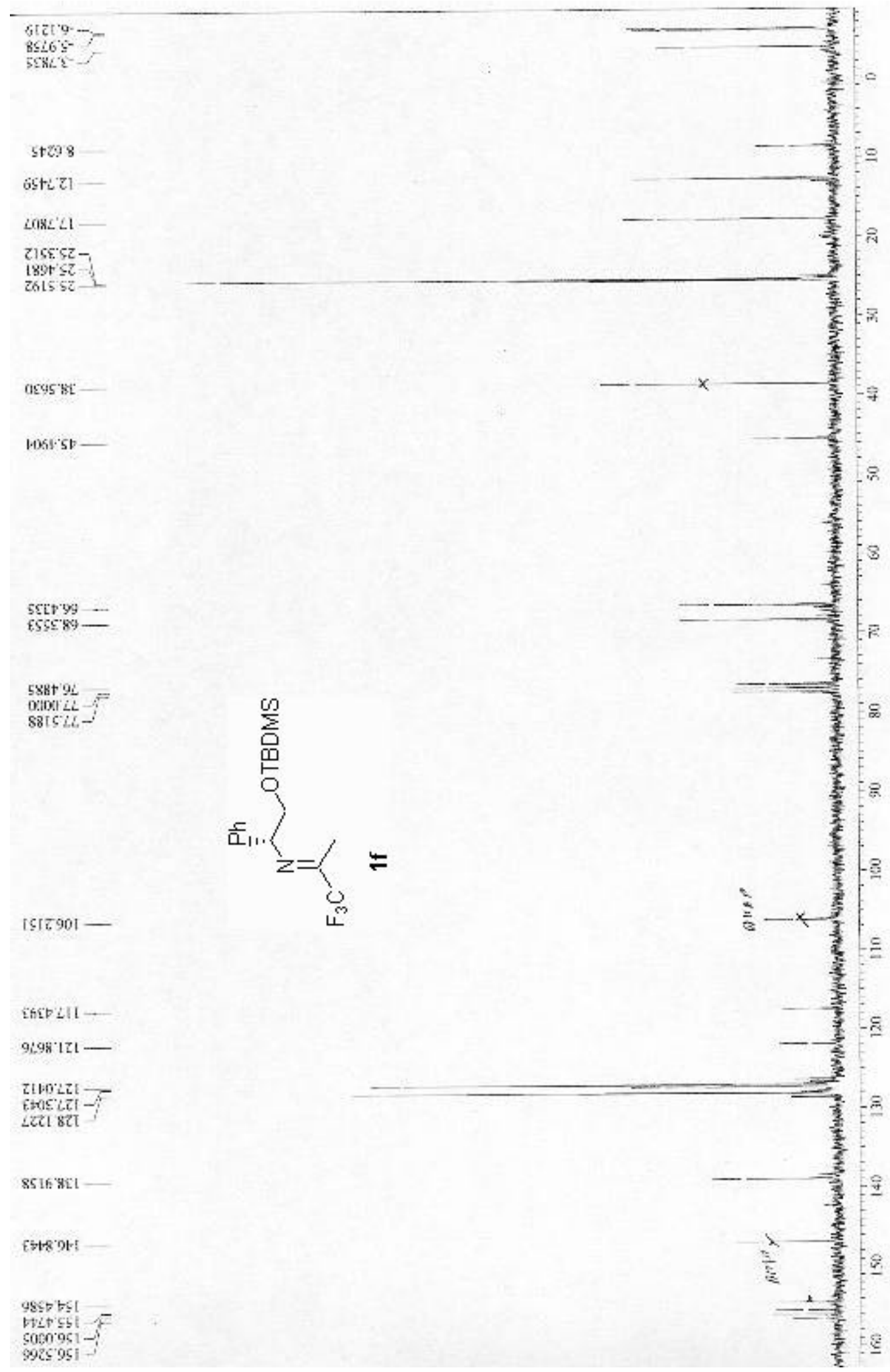




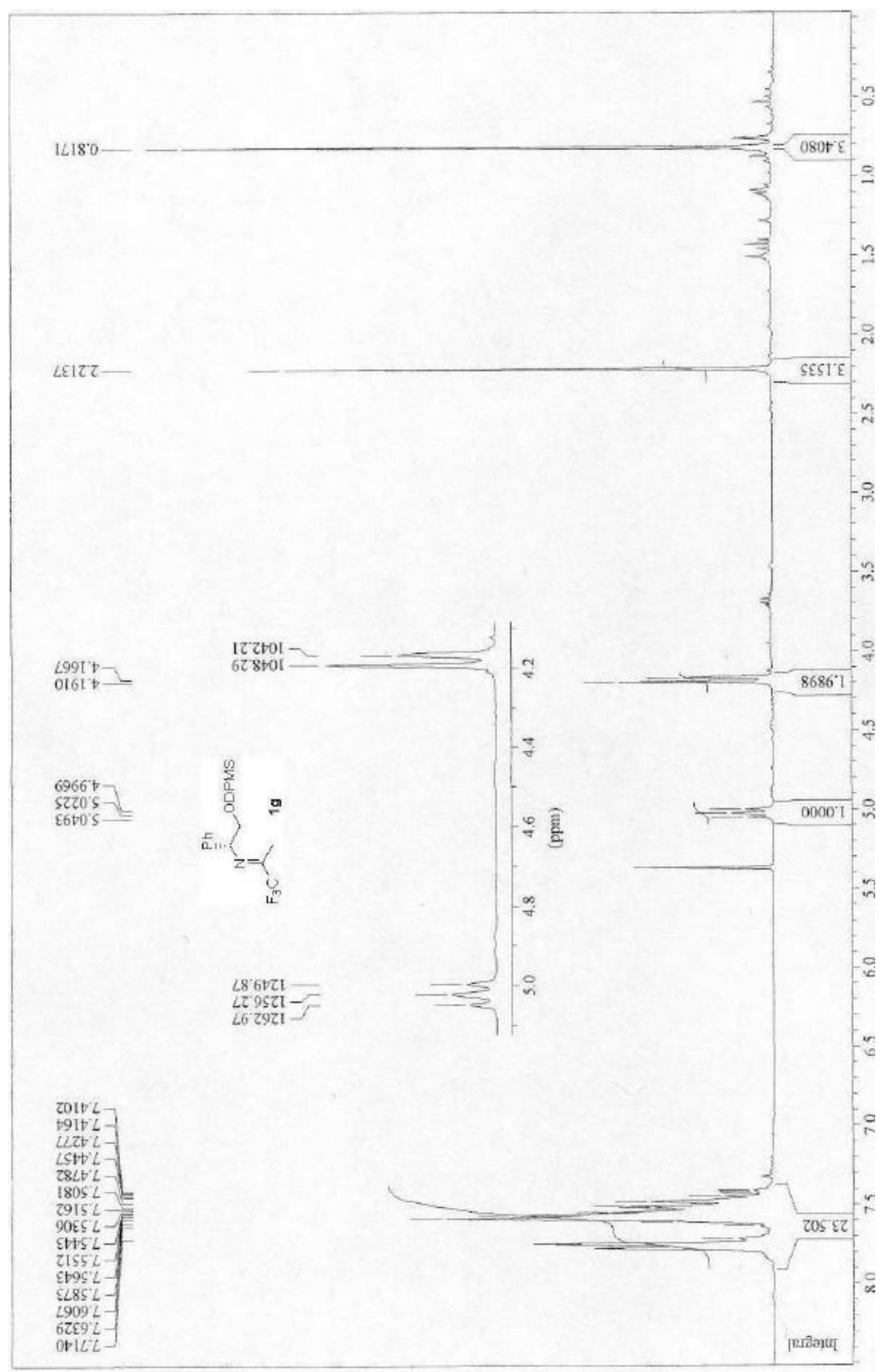




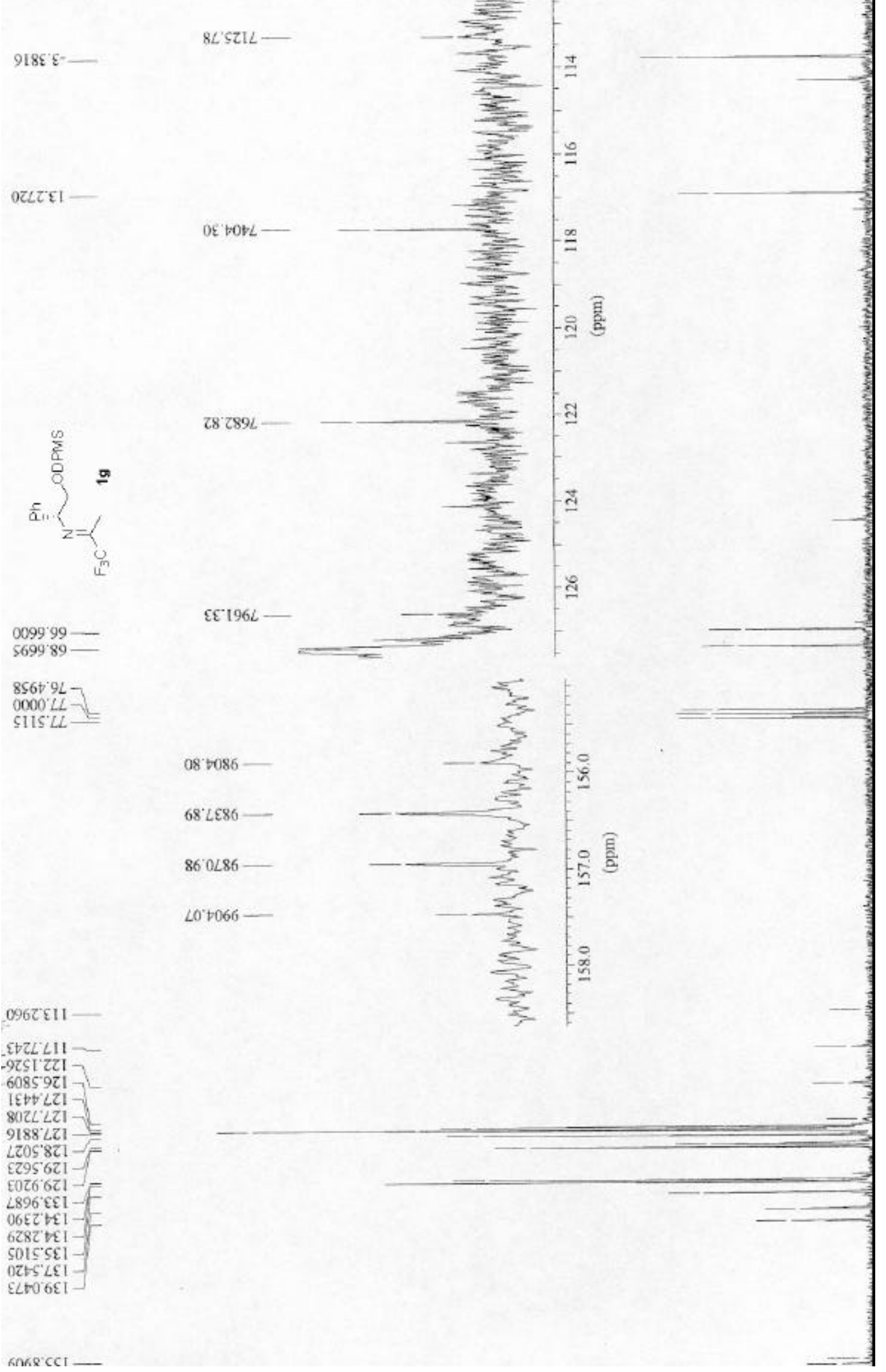




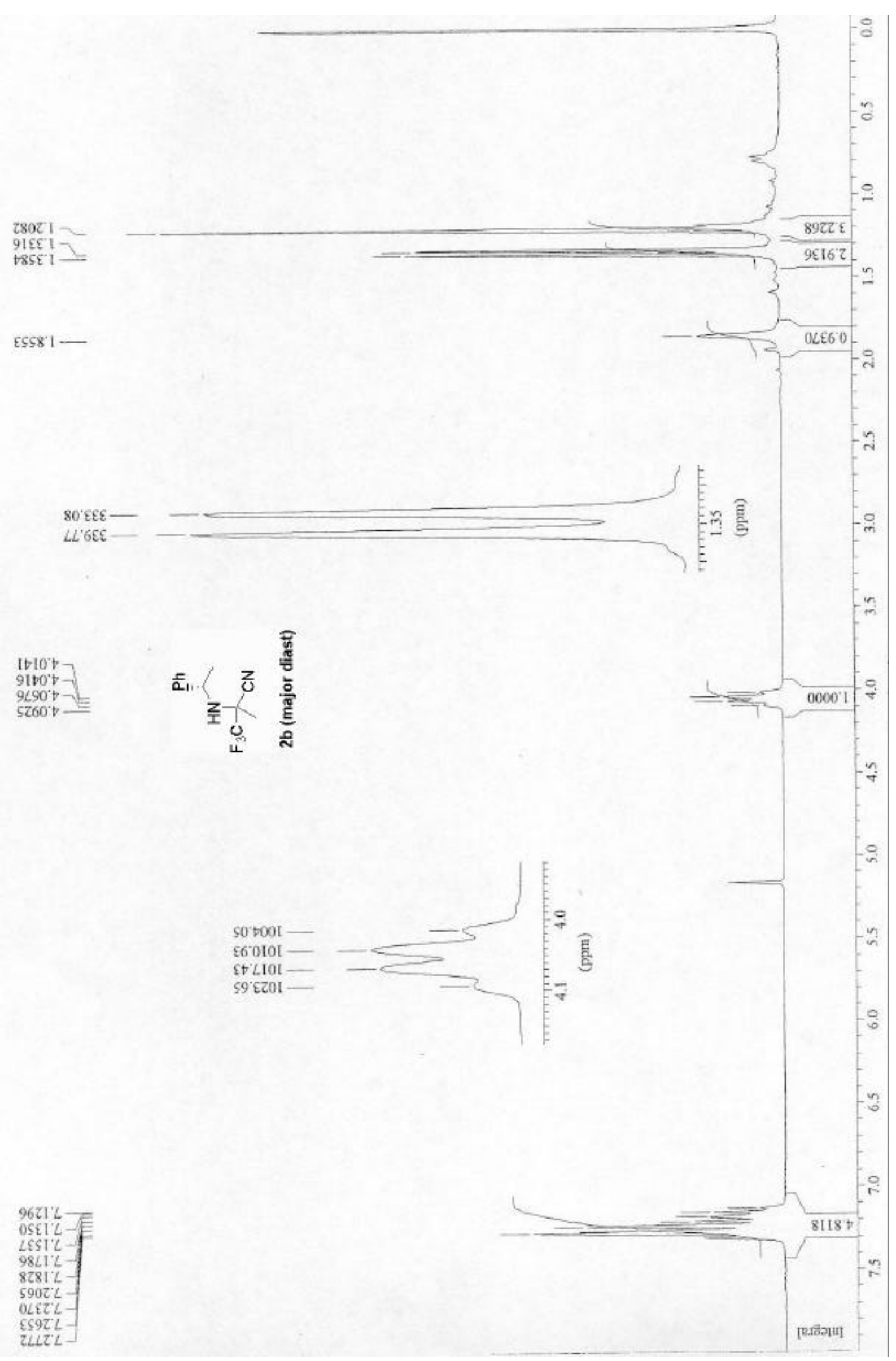




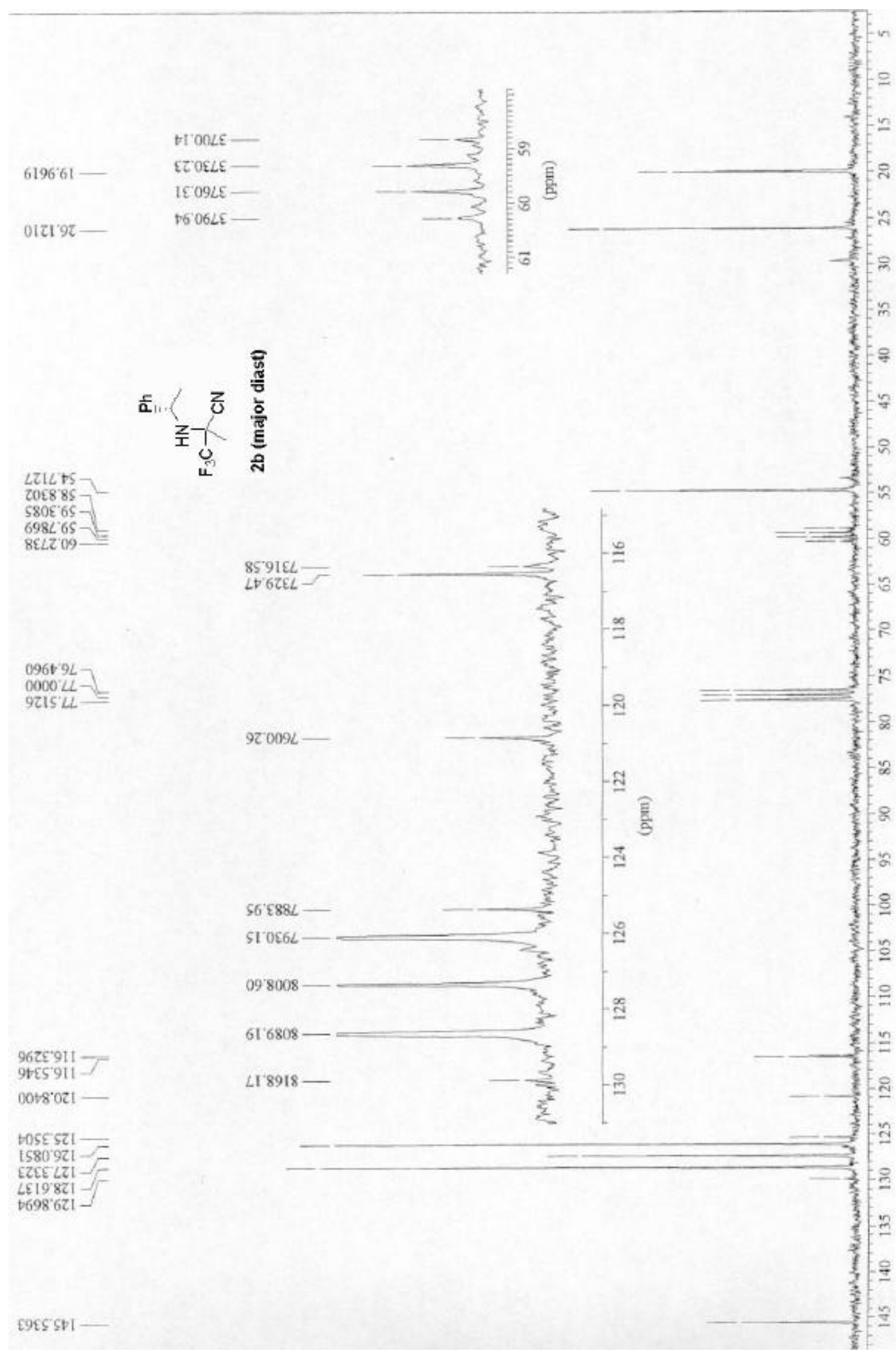




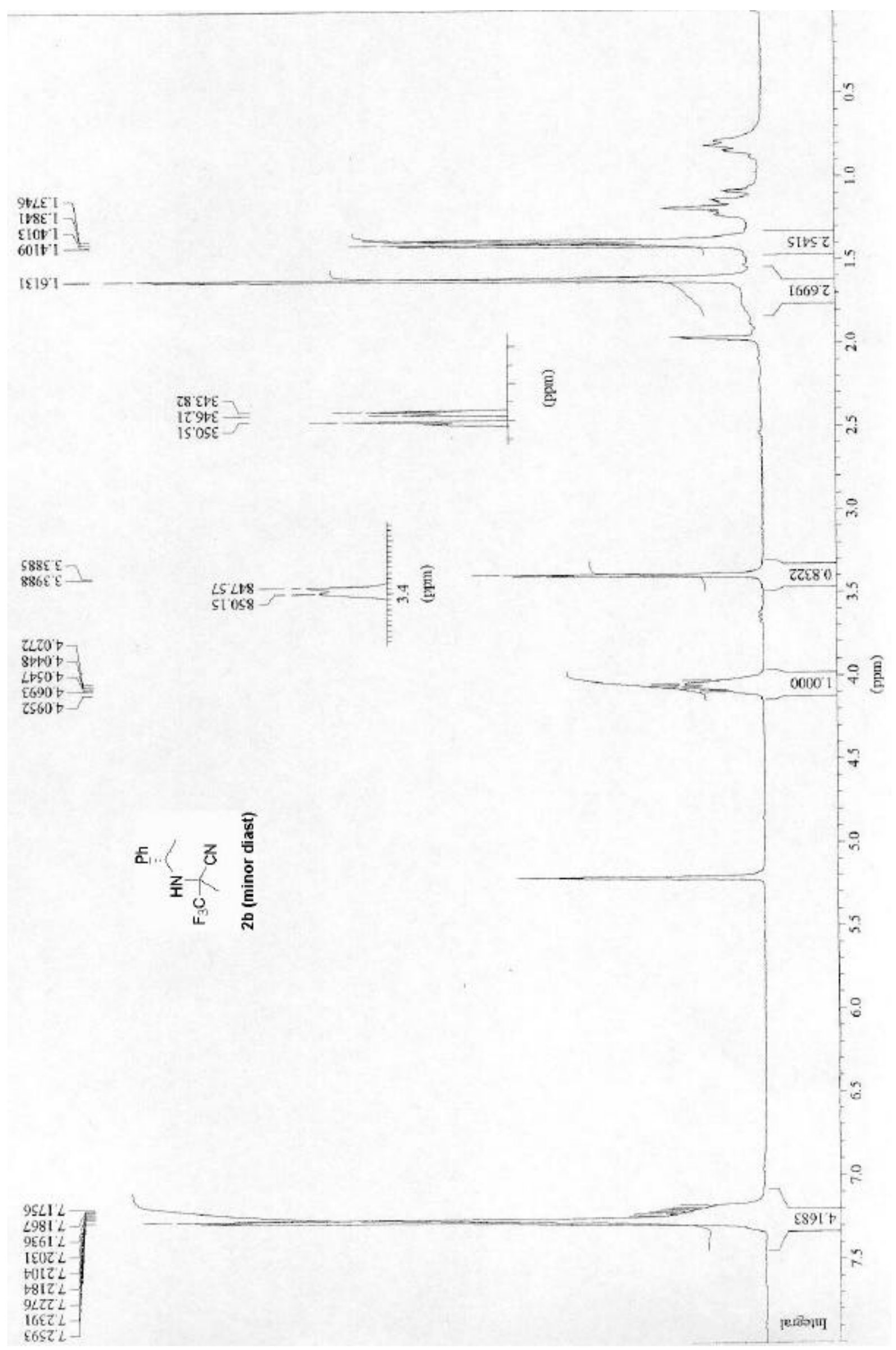


Lazzoz -
octs sz

t9st $t 5$

$860 Z L S-$
ZStLLS

żะz $8 S-$ y

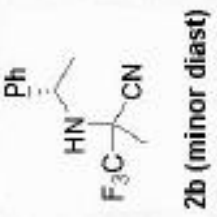

$t \angle S+9 L$

$00002 L$

tLooge

O5 $1 £ 98$

ES Z99E-

ร1 $\varepsilon 69$

(1T) LL -

$9628911-$

$2+\angle S O Z I$

zolt $c Z 1-$

s9et

0965821

LIE6 621

$65566^{\circ} \mathrm{ET}$

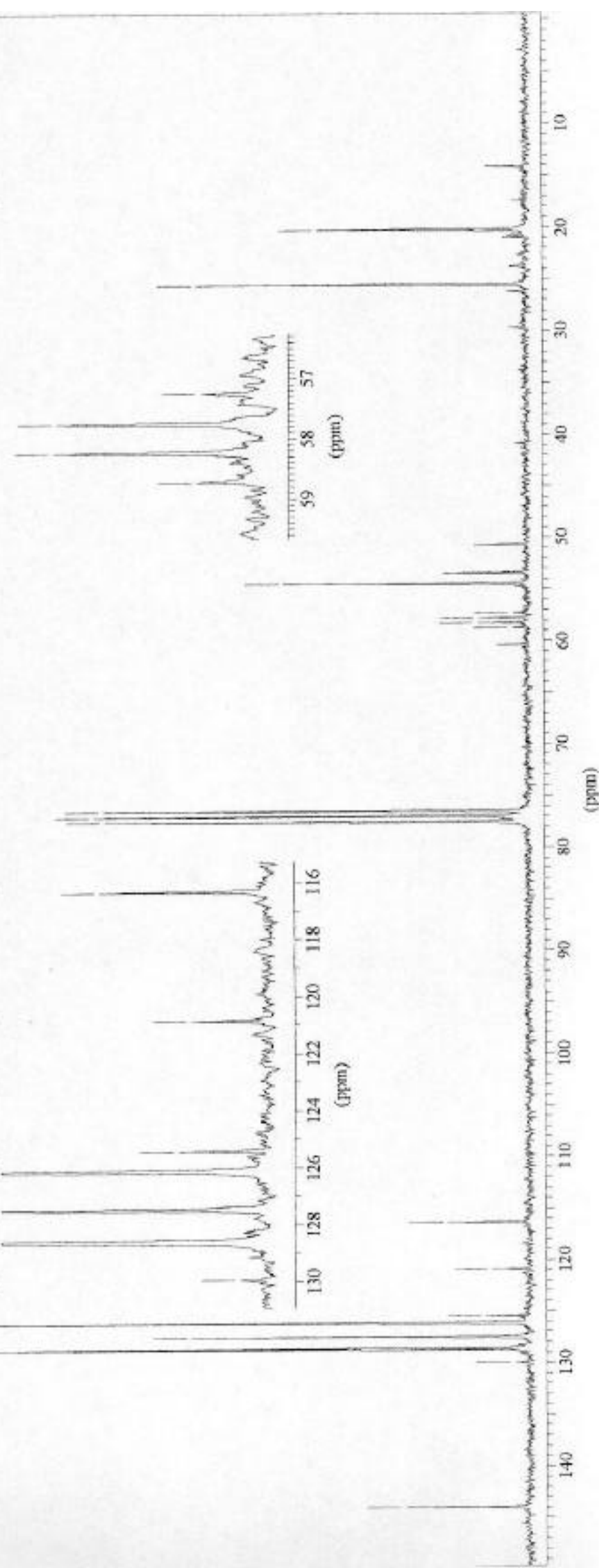


Lazzoz

octs \รZ-

t9stits

$86+\tau$ LS

TSDLLS

I61485

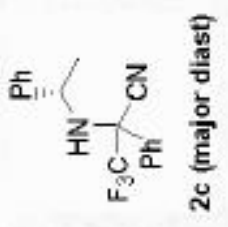

†L 0098

05 1 1 95

ES Z99E -

ร) E69:-

$00002 \mathrm{LL}$

(1) $15 L L-$

968 $911-$

$2 t / S 0 Z L$

Zolt' $c Z 1-$

s981 $921-$

$069+2 \mathrm{Zl}-$

LEE6 621

6556851

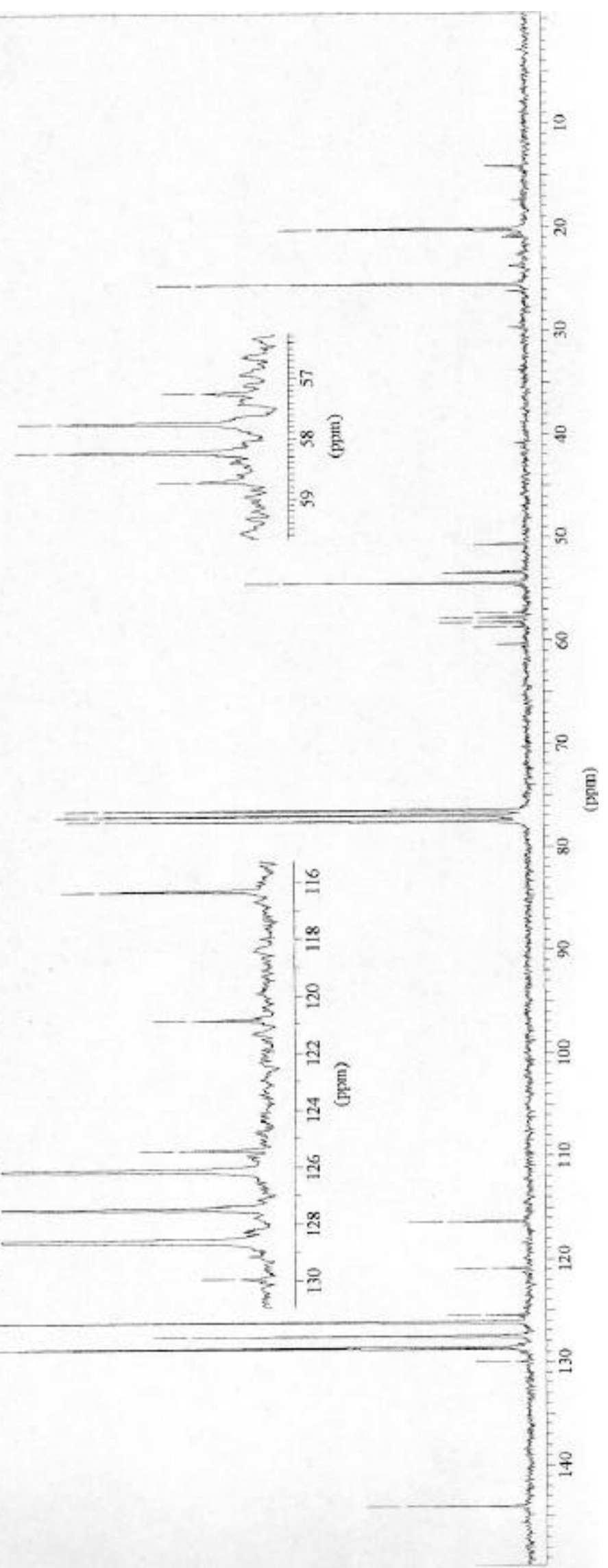


$112+62$

E\&EL t $\zeta$

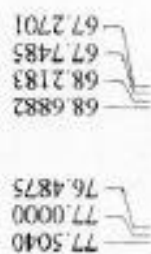

0 otos $L$

7089511 अㄹ․

potzozi -

$; 2 S L \mathrm{NZI}$

in92 $921-$

:226 921

1602821

$15+821$ -

Z2EO 621

882621

$0<2$ '0e1

$899 t+61$

$$
\begin{aligned}
& \text { L6 08zt - } \\
& 90 \text { 19zt - } \\
& 19062 t- \\
& 91 \text { ozst - }
\end{aligned}
$$

$$
\forall L S L Z L-
$$$$
\text { 859IEL - }
$$

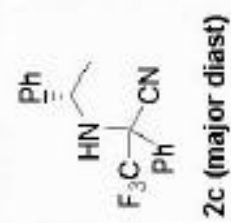

to' T9SL -

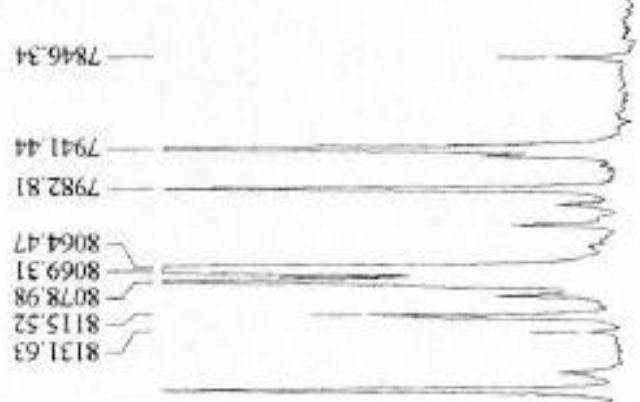

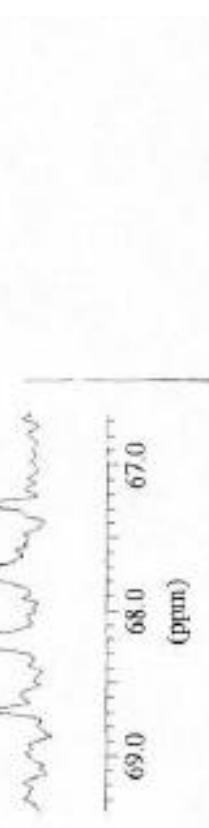

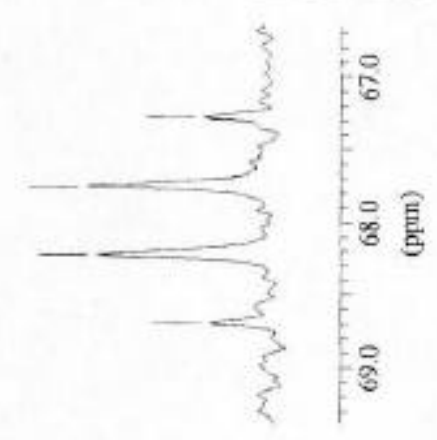

요 


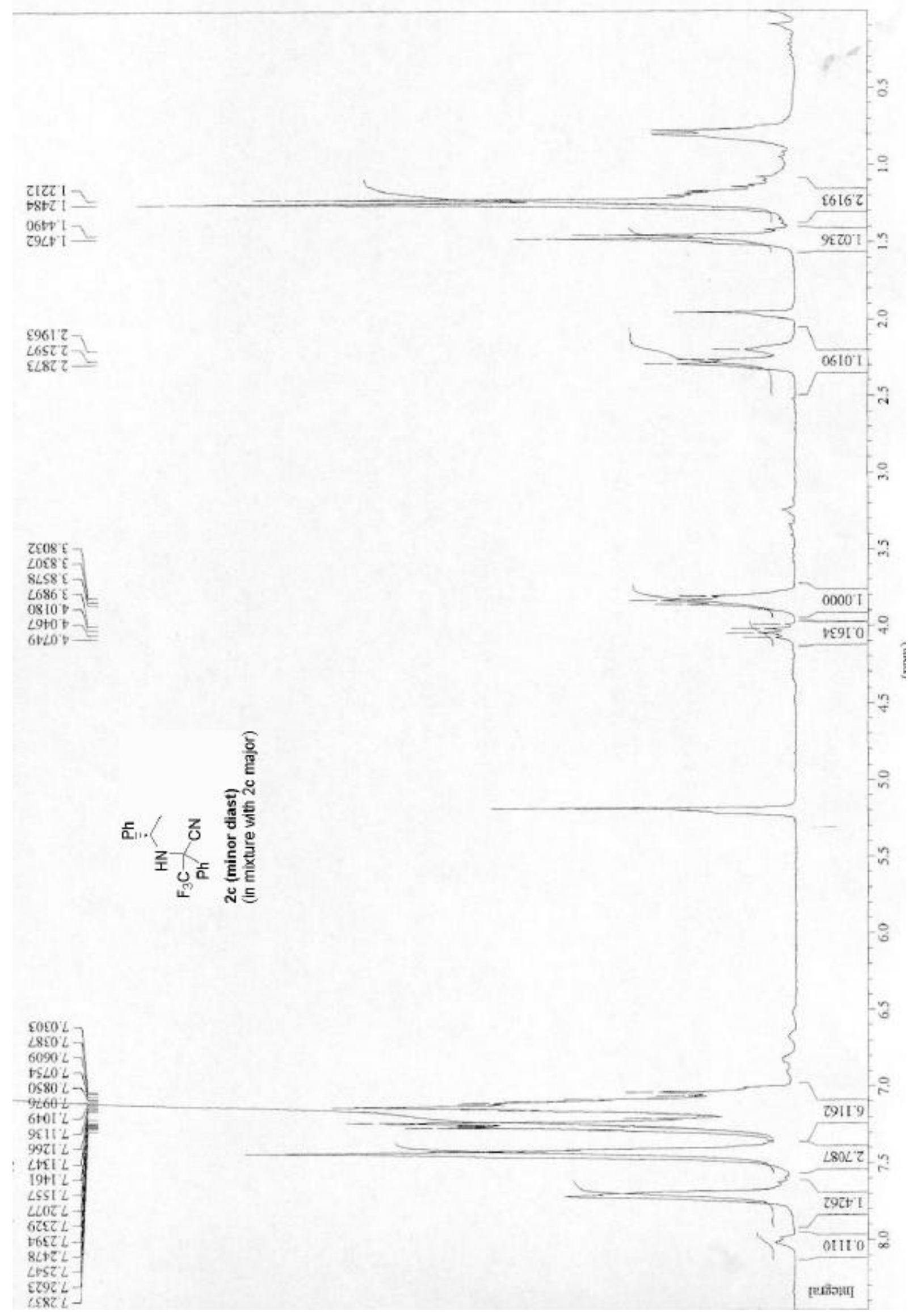


£219'99$\angle 060 \angle 9$

096796

$0000 \mathrm{LL}-\mathrm{L}$

$S 21 S L L=$

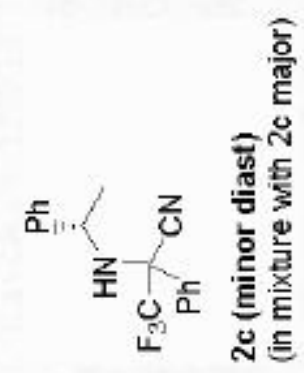

9EZL

ZS5E' 91

16 STOZI $_{-}$

$\tau \$ 6 L \triangleright \tau \mid$

$8+2 \varepsilon s 21$

[062921 - F

7945921-

$6096921-F$

$6198 \mathrm{LZI}-$

$6+52821$

z¿zesz

ss8t 821

E6E9 8Z1

\$ $688^{\circ} 8 \mathrm{Zl}$

IIIZOE I

ISOE OEI

$89+90$ ㄷ․ 


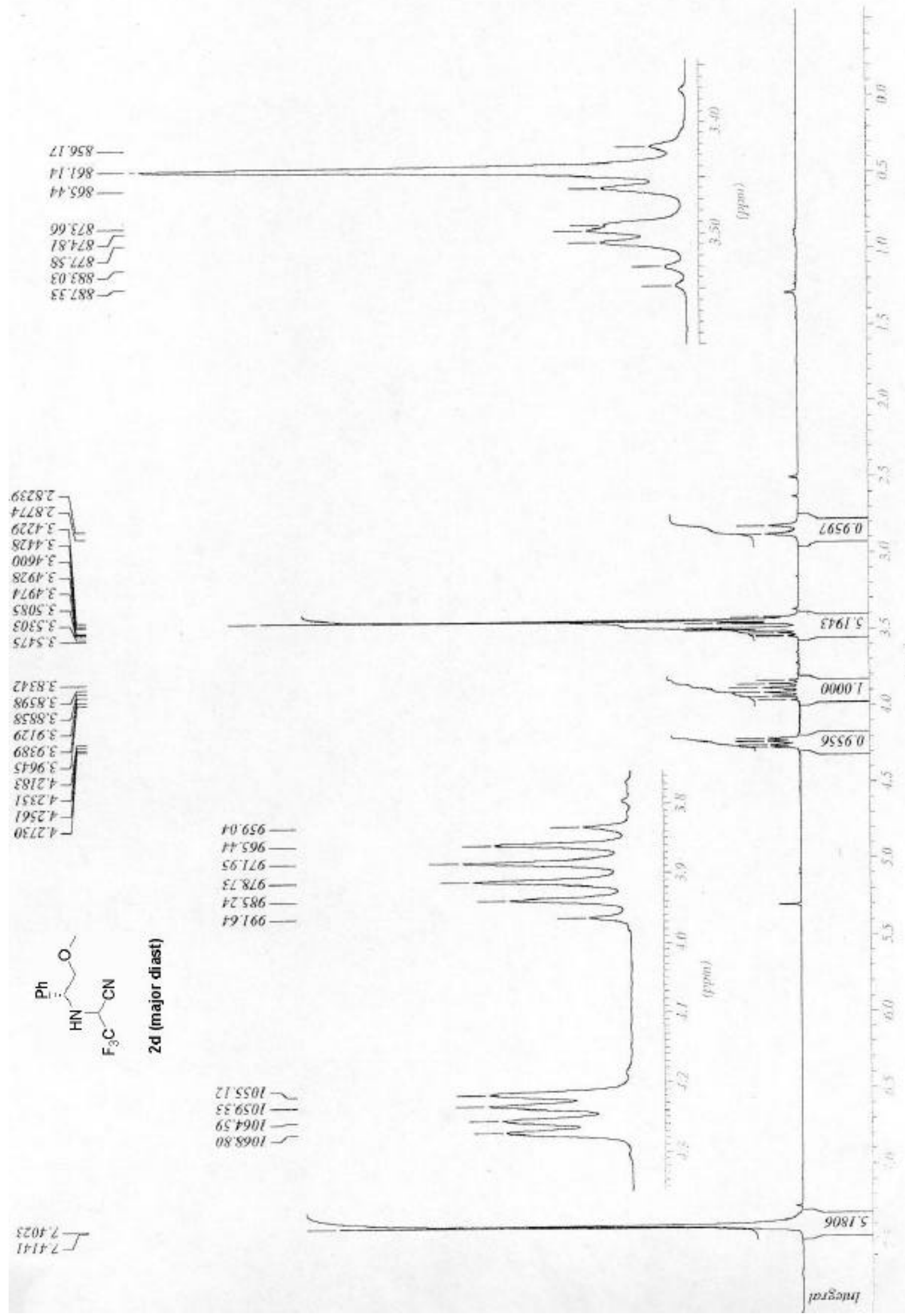


$09 \angle E I \varepsilon-$

ESZLIE-

$16902 \varepsilon-$

OE' $7+2 \varepsilon$

299865

tittios

6tes 15 15

LEE9 85 -

44665

$9 \angle 2+92]$

$528+92$

oros $\angle 4=$

St $2 Z I L-$

$\angle 00 E Z L-$

$\operatorname{Es} 0 / S<-$

910 0624

gatereII-

$2+56 \% 11-$

HEIF $6 I I-$

op9s"Ezl -

$6959: 221-$
$0 \angle 88^{\circ} 821-T$

$9[1[621]$

9052951

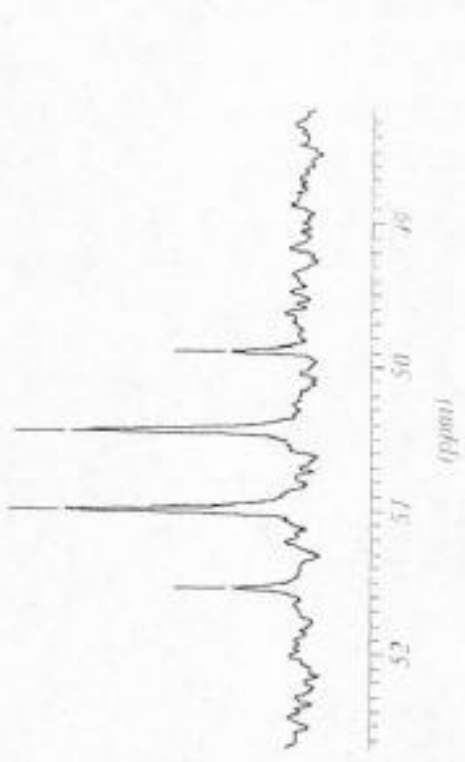

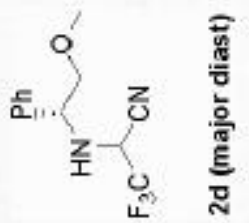
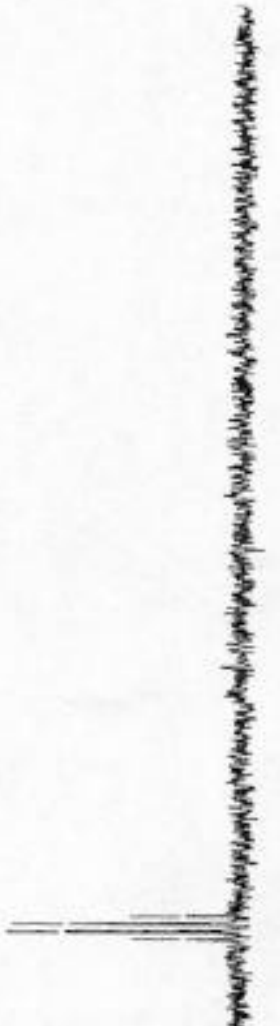

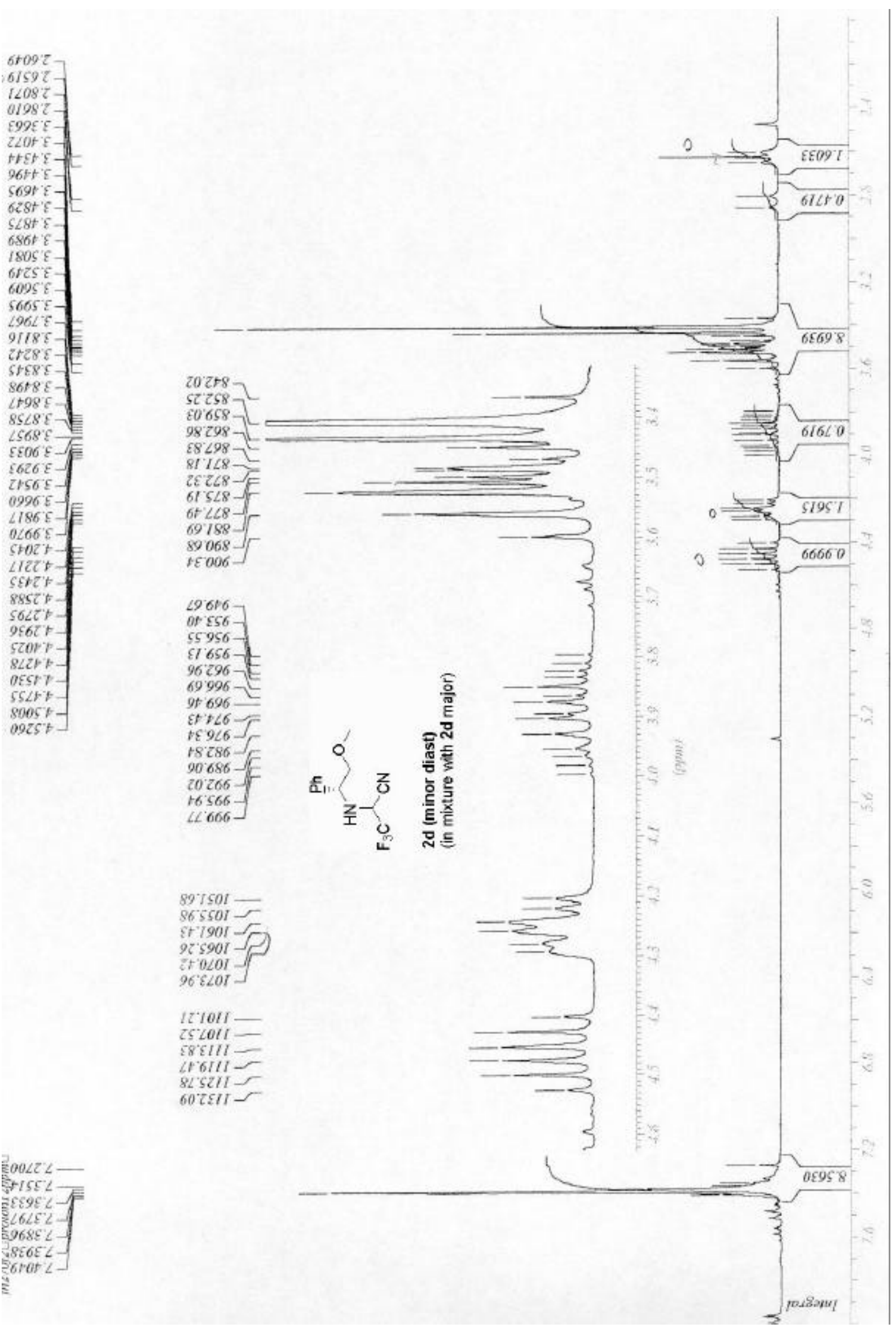

B $00<2 \angle$

St+15:2 -

GeEge L-

$5 \angle 6 \angle E L-$

$5968 \mathrm{CL}-$

SBE $6 E L-$

5 rotat $\angle-$ 


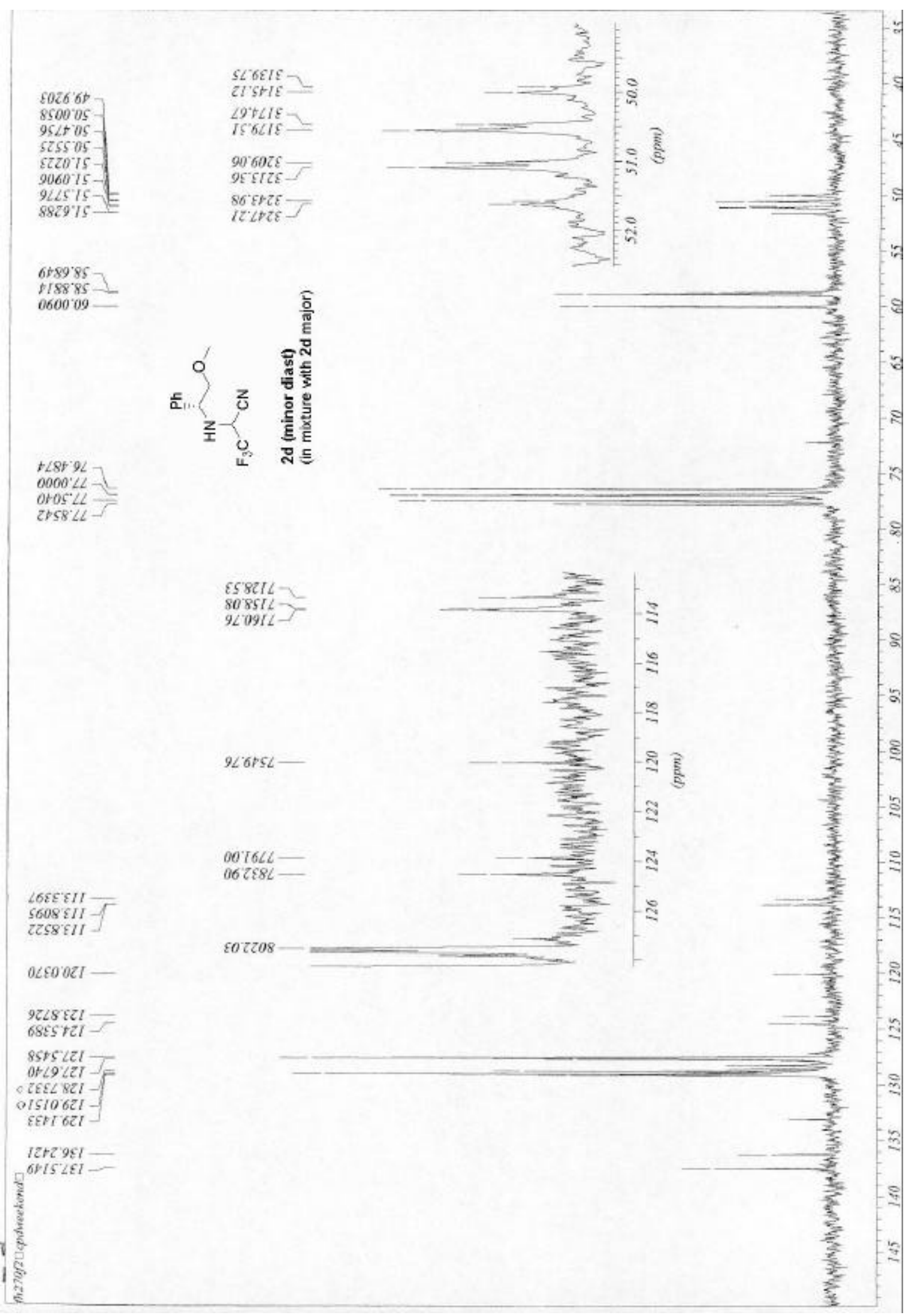


$6\llcorner 650-$

ESDI'L -

LI80'
06. 266

169660 ş 200L LS 9001 -

$58906-$

$9+216$

LS. 126

50662

zlot $\varepsilon$

LIH ₹

$5529^{\circ} \varepsilon$

๔ZP $\varepsilon-E$

$6299^{\circ} \varepsilon$

$6489^{\circ} \varepsilon-=$

$5696^{\circ} \mathrm{E}$

$9586 \mathrm{E}-$

$[800$ ' - -

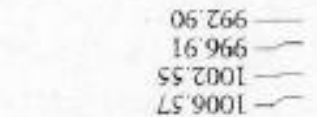

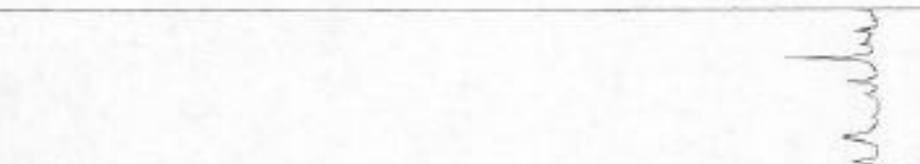

$\rightarrow-89$

9862
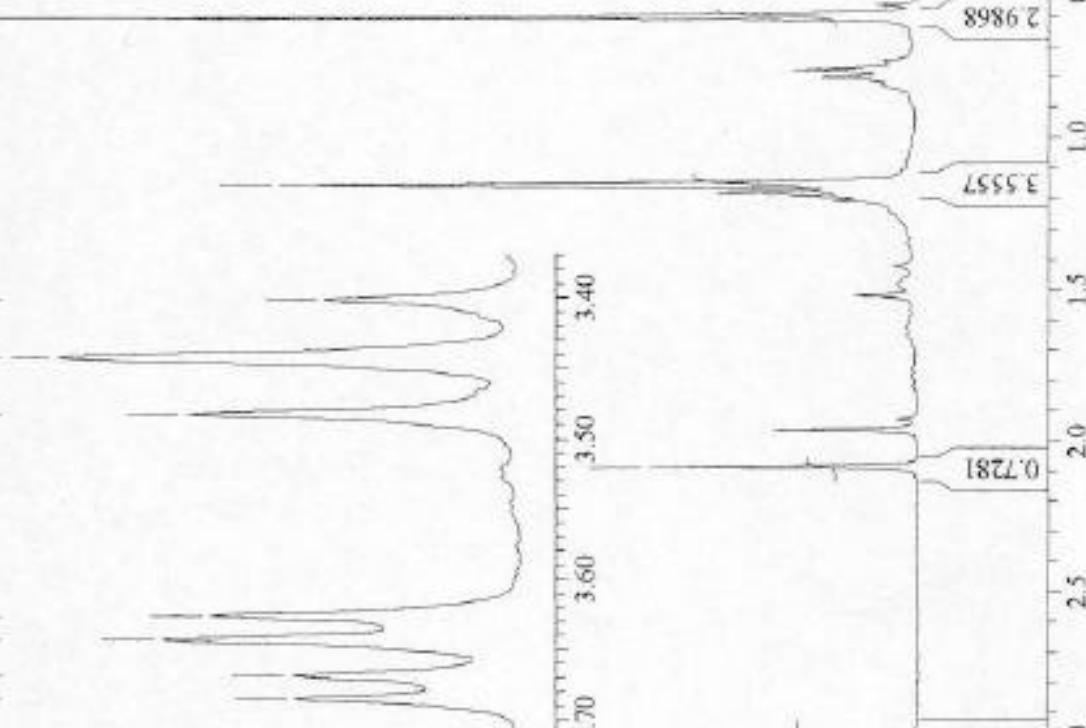

策
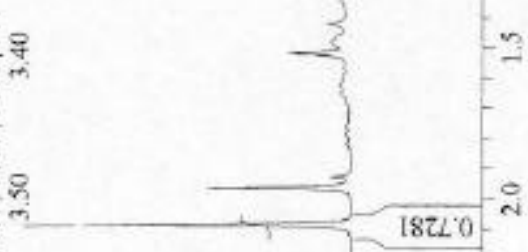

豆

$-\infty$

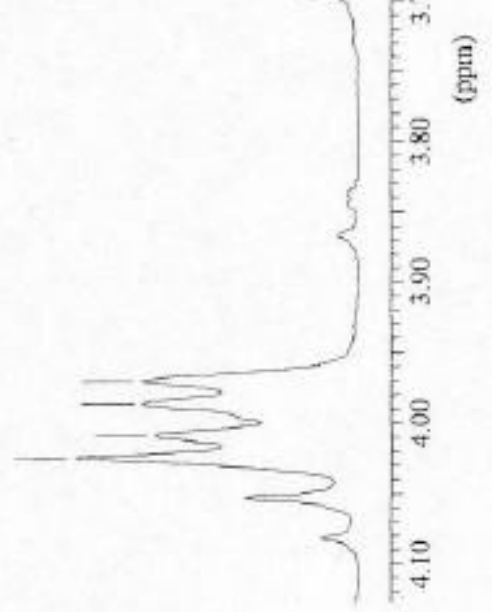

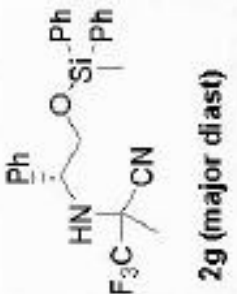

6ISIL

EELTL -

E961 $L-$

$\angle L I Z L$

$85 L 2 L$

$2862 \mathrm{~L}$

WOOEL

EITE L

CgcE $L$

99eE 2

$909 E L$

SSLEL-

O+BE L 
sGะZ \&- -

zsะzoz-

to 65 . $65-$

$26+665-$

9L2t 09

$\left.\begin{array}{l}5+1609-1 \\ 564119\end{array}\right]=$

$286 \varepsilon<9$

$2607 L E-$

tsoLCE-

$19008 \varepsilon$

Eะ IE8E-

$68 \angle 18 E$

$096+9 L-$

s215 L

Lร9E 911 -

\$2t60zI -

S19t'SZI -

$\angle Z 1 Z \angle Z 1=$

9866 LZ1 -
S6LC 821 -

S6LS 821

86SI 0EI -

$0016 \mathrm{EEI}-$

Z092 bEI -

EILE DEL -

Itto'tel

₹986 tE 1 -

$\angle E O E O D I-$
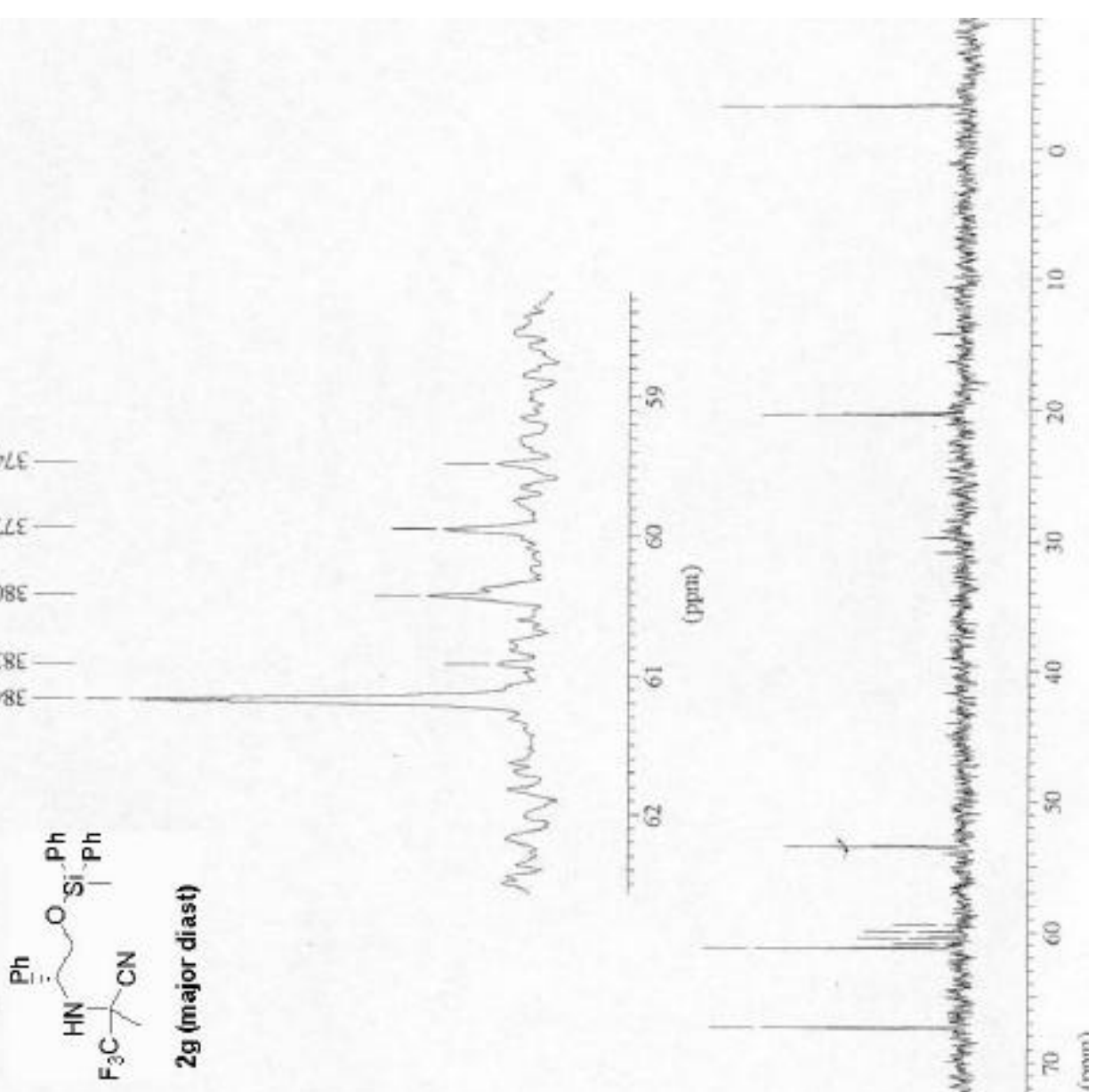


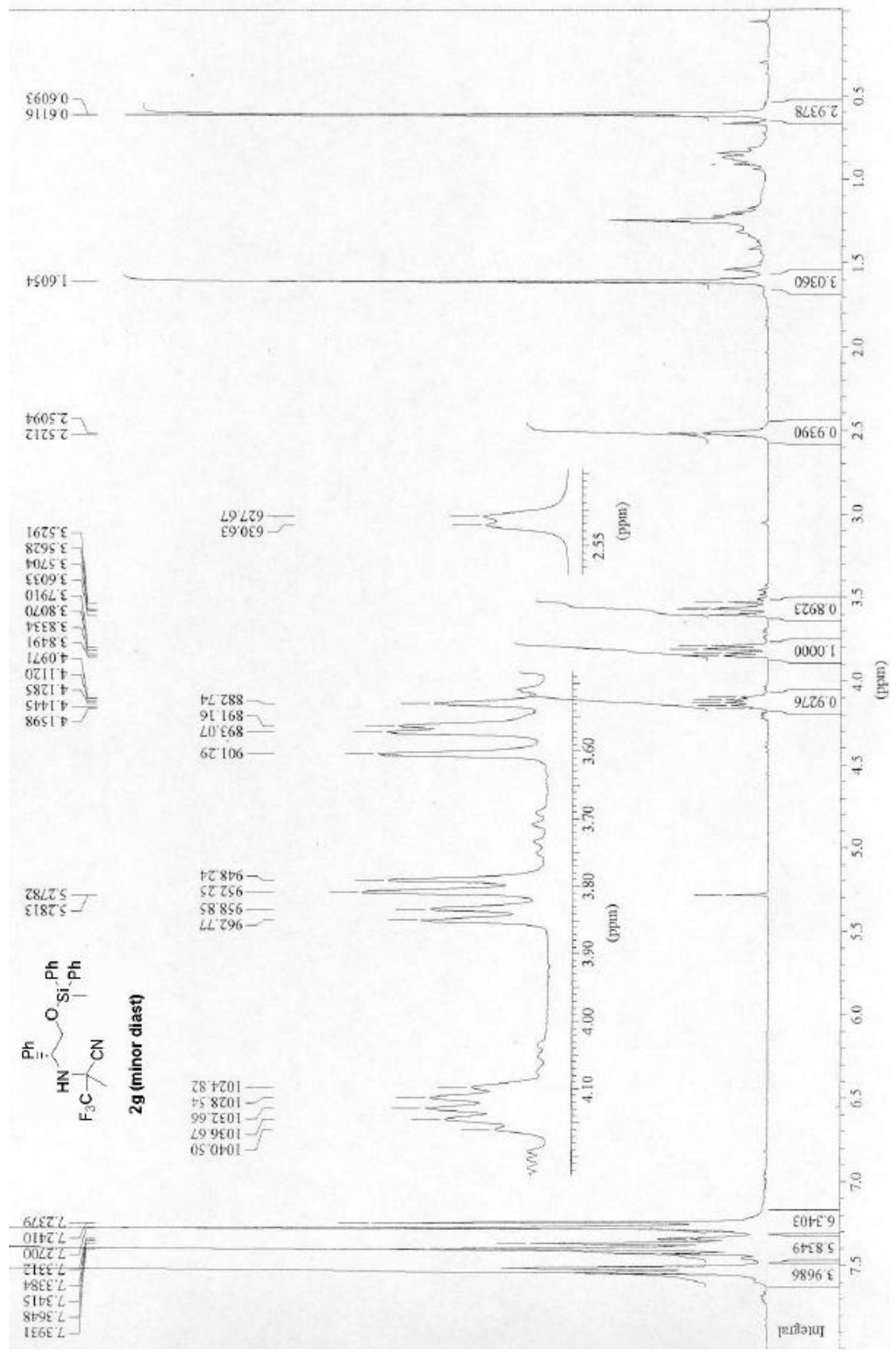


aztse-

$98160 z-$
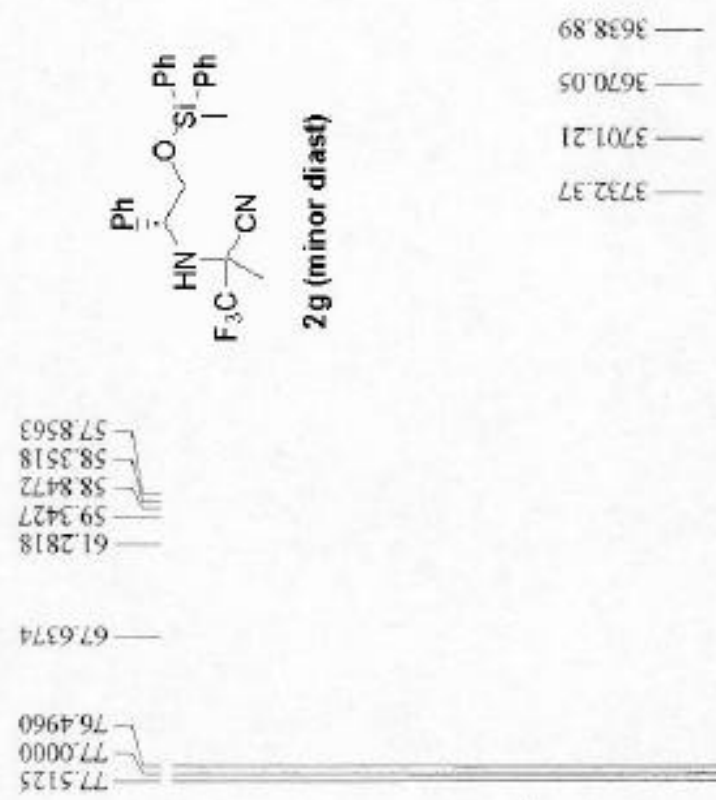

IEEI 911

sてाย9ाI -

$95588 L$

$00+8021$

$9 z 009 L$

19LESZ1 -

$\mid$ troz $\angle Z \mid-$

$9669 \mathrm{Lzl}-5$

$z+20 \mathrm{sz1}-5$

$190 \varepsilon^{8} 8 \mathrm{zl}-$

$86510 \mathrm{OLI}-\mathrm{F}$

$889 \tau+t \varepsilon 1-$

- sozoset-1

- SOZOSEl -
cosose I

.82186 6I
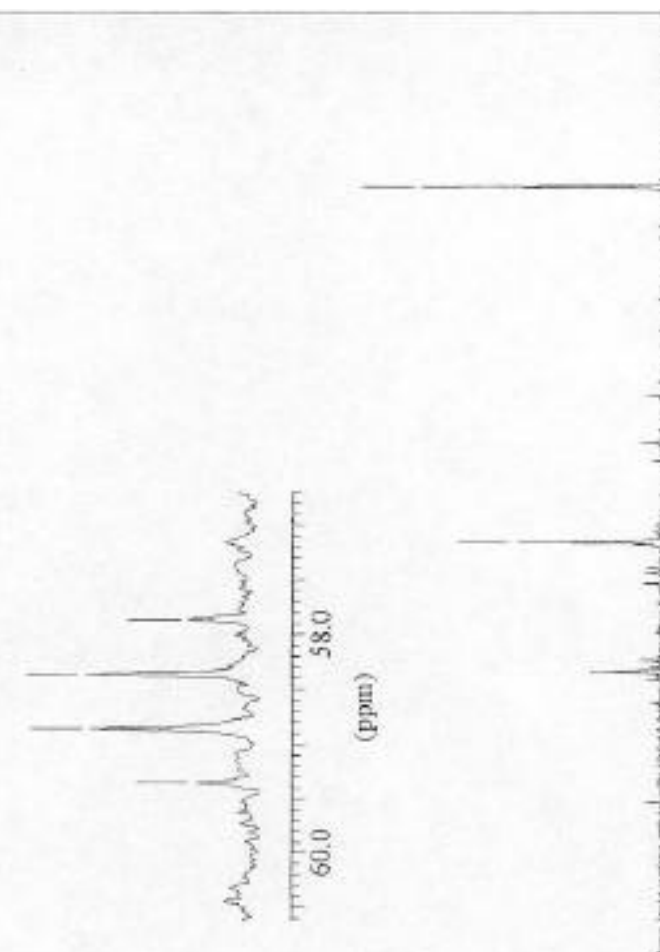
$Z Z$ bOEL
OS SIEL

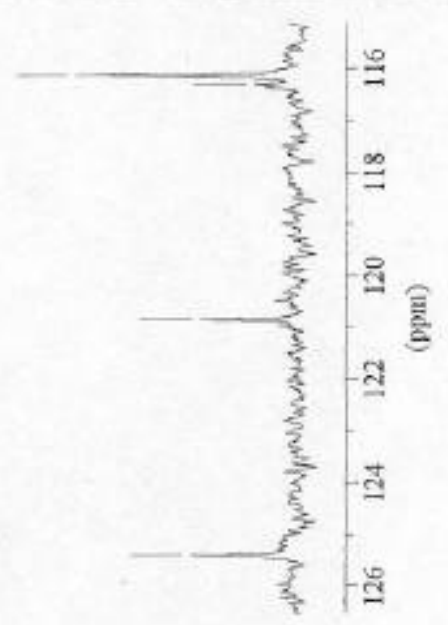




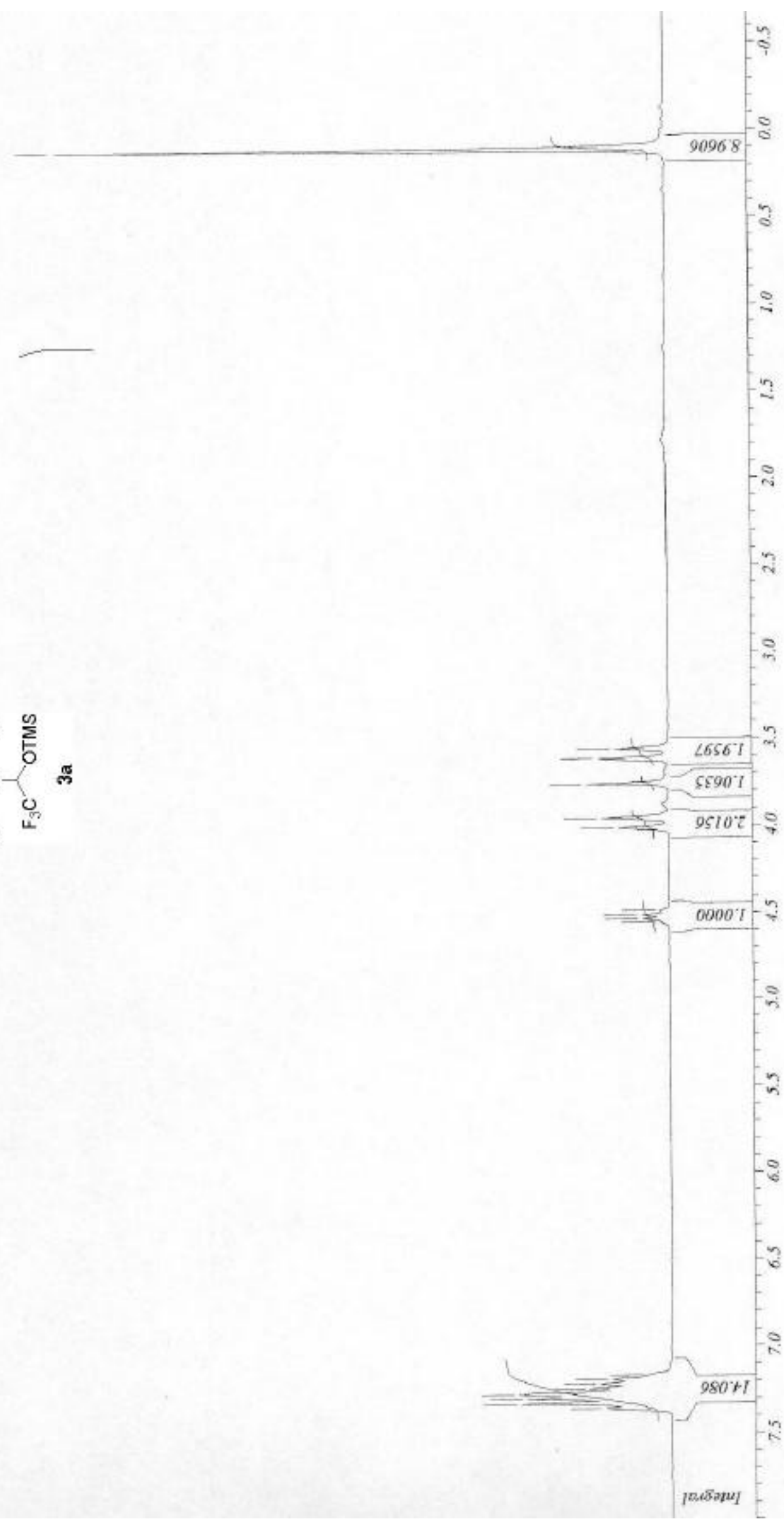


SE $\angle E O S-$

86.6905

lozols-

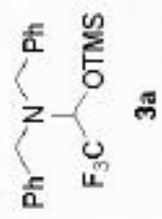

stit's -

$856+92$

$0000 \mathrm{ZL}$
SIIS $\mathrm{ZL}$

aroover

$660908-$

$\angle 82178$
$S \angle 978$

2180211

$\angle E E 9^{\prime} I Z I-$

68:I921-

$\angle D 6 I \angle Z I-$

sose'sel -

5879 827

$6 E 0 \angle S E I$

$\$ 8 \div 9 \varepsilon L$

810592

909562

106664

$208 \angle 08$

E67228 -
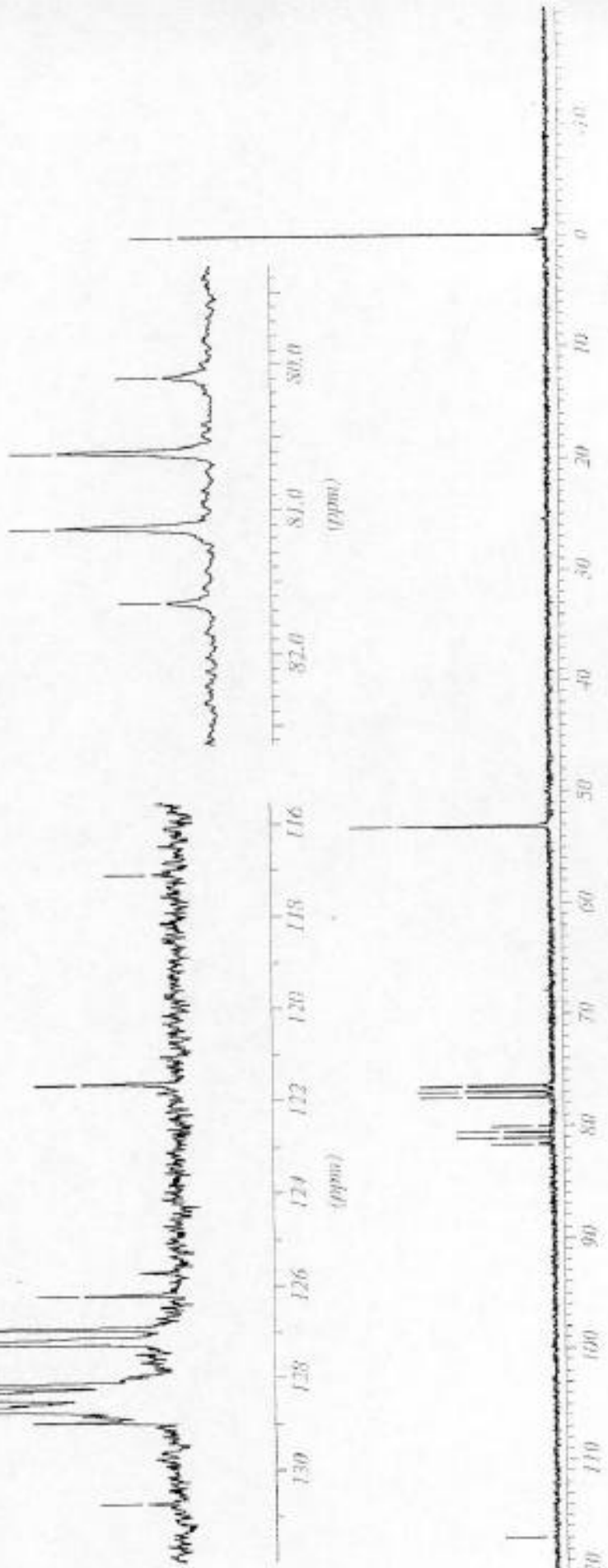

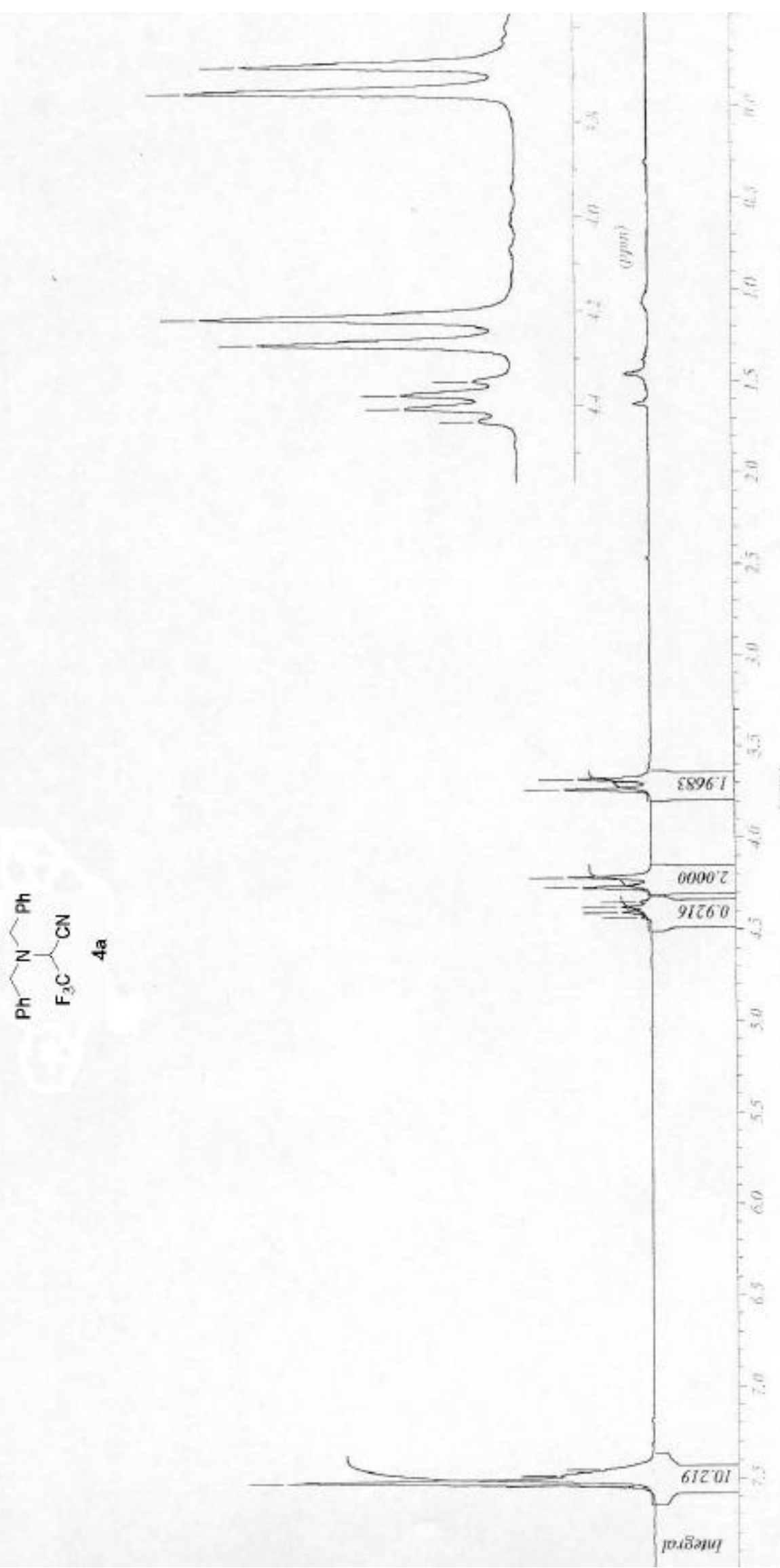

$+25+2$

$56 C t 2-F$

$1+8+2-1$ 
oz9ots -

szosts-

$55+155]$

Et89'ss

682095

C8SP 92

$0000 \mathrm{LL}-\mathrm{L}$

511542

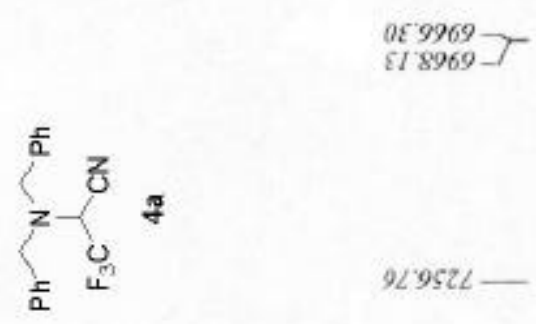

s092011
sos ool $]$

98LE'SFI

$66 \angle 8611$

EISE't

$91 \angle 0821-$

$5899821-F$

$612 \angle 821-$

$0080621-$

$5+21951$

댕

602258

886852

662282

60 S508

दहco0s tevots -

$c c+\varepsilon+\varepsilon$

928952

827058
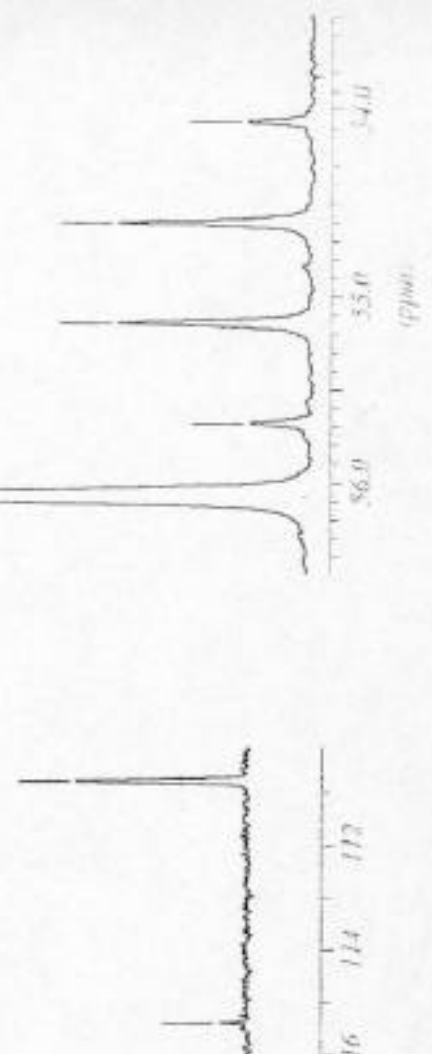

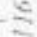

는

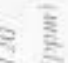

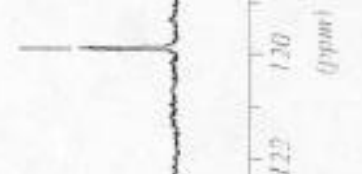

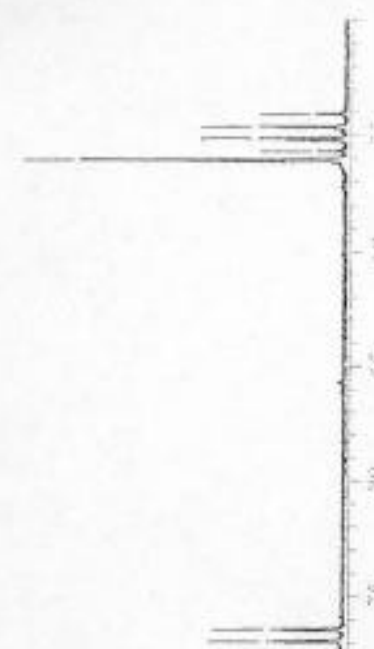

,

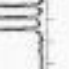

$$
\text { \% }
$$




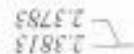
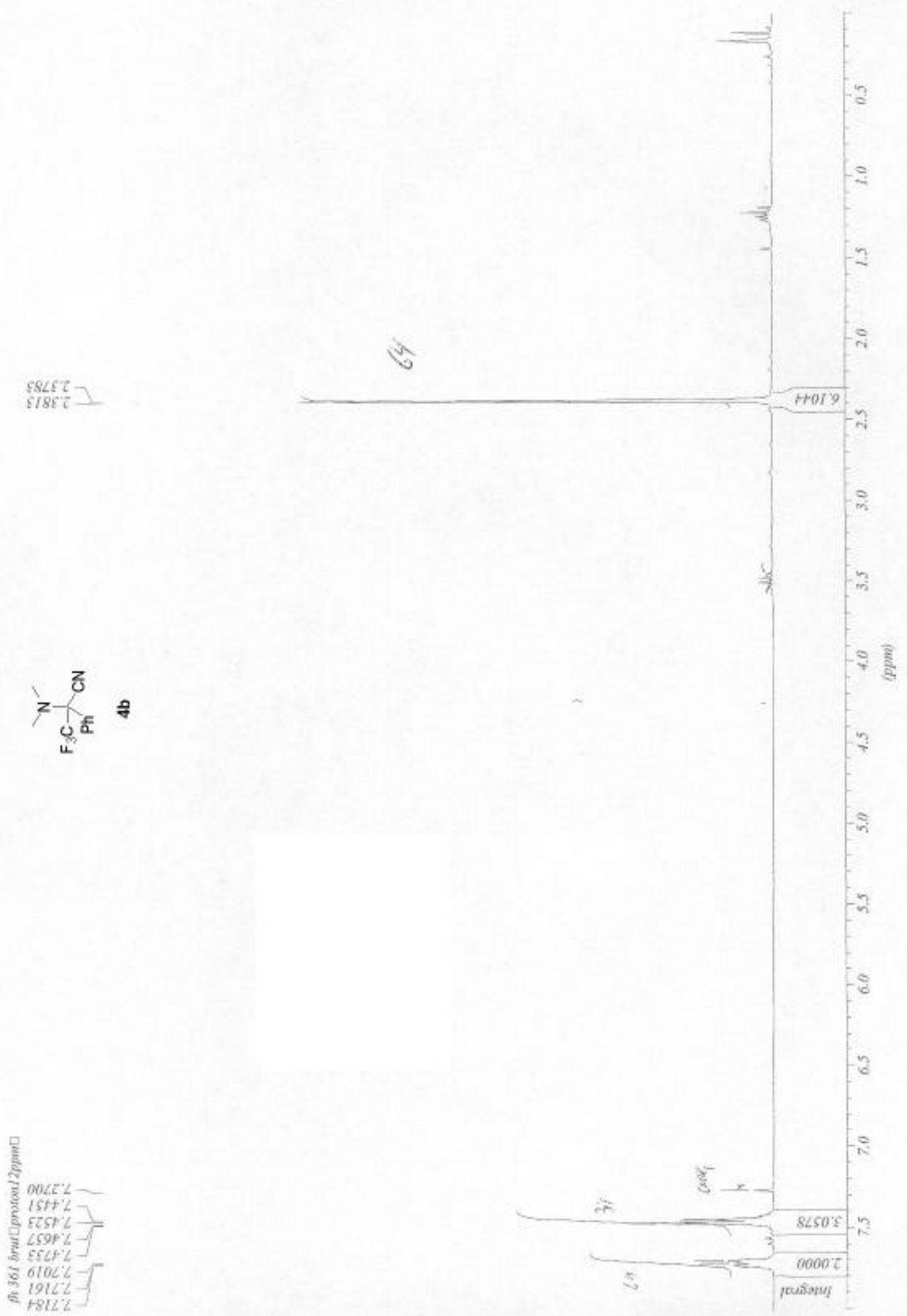

$z-\sum_{\substack{0 \\ i}}^{z}$ ㅇ 


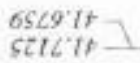

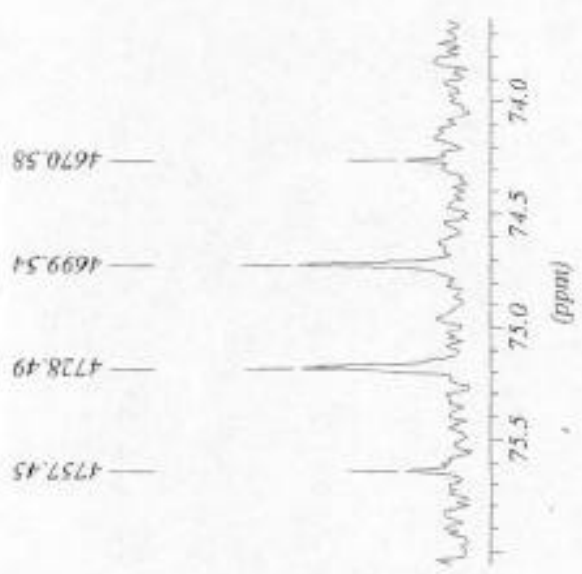

$\angle 6 S Z^{\prime}+1$

roEL Th

$508 I^{\prime} S L$
$80 \mathrm{r}^{\prime} \mathrm{SL}-$

$5855^{\circ} 9 \mathrm{~L}-$

$00000^{\circ} \mathrm{LC}-\mathrm{H}$

Ctos $2 L$

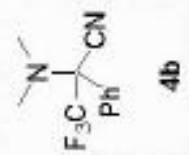

chest
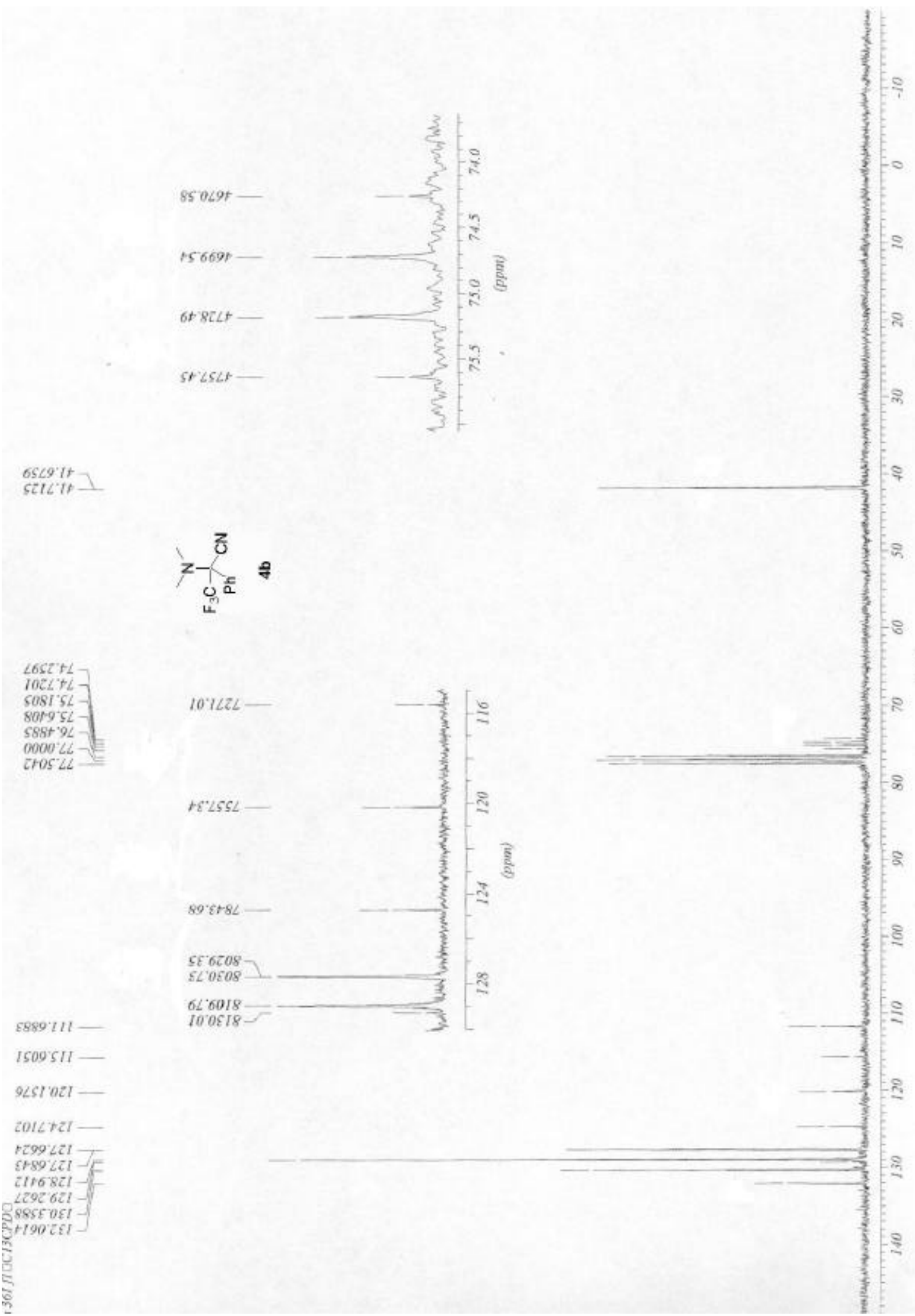


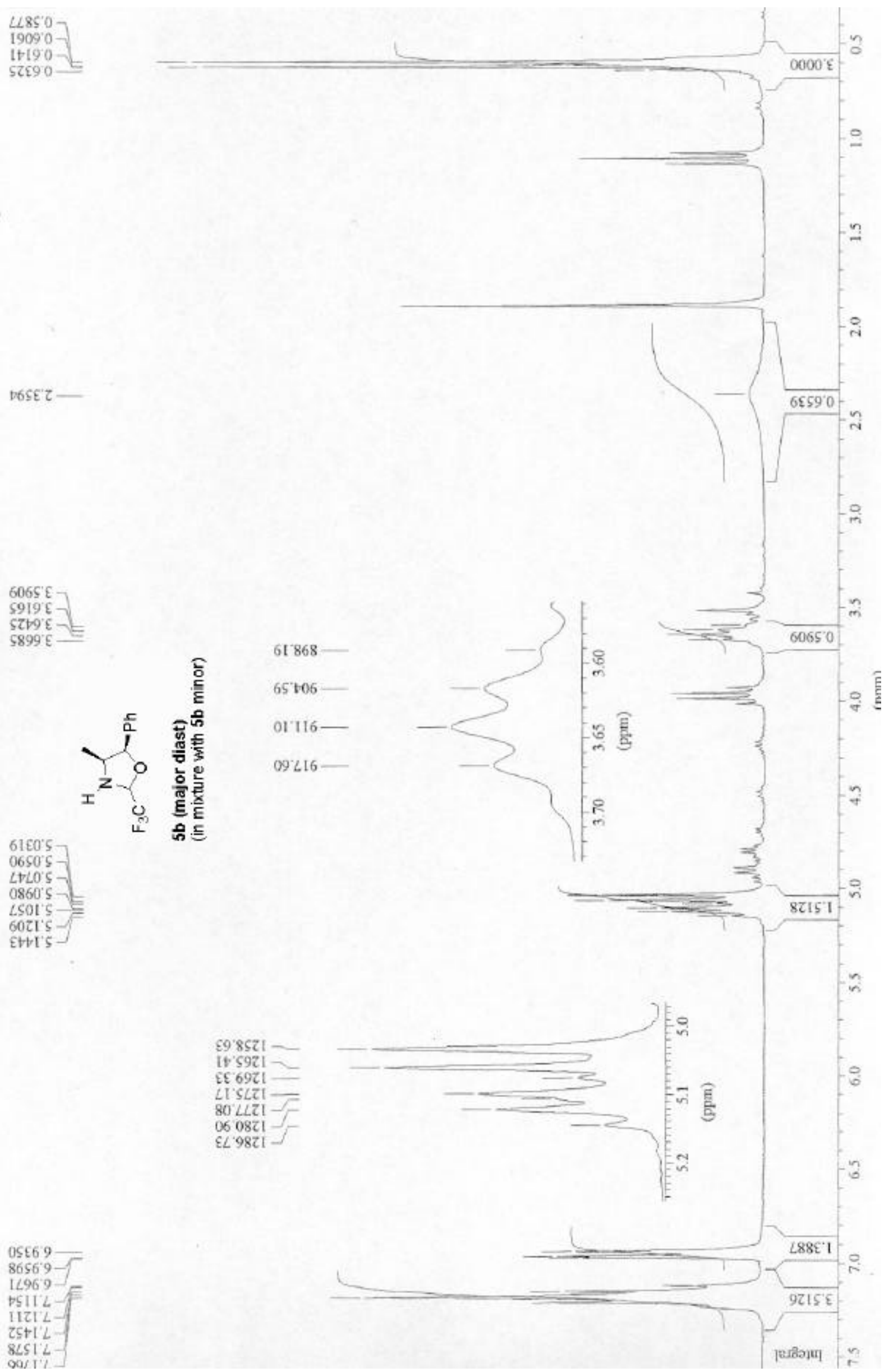


IEZZSI -

2L6291-

SIL09S-

$8 S L S 95$
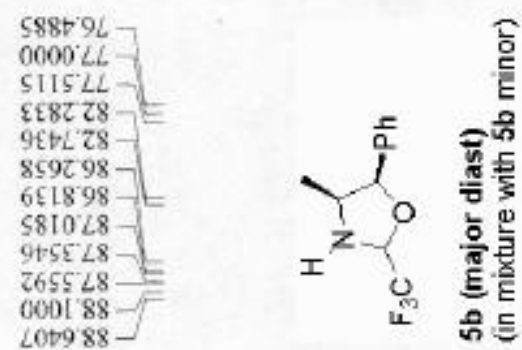

8259911

tISITZI -

1099 szI

zट26 szl-

zा60' $921-$

ธ\ILLZ1-

$6\{6 L L Z 1$ -

$5881821-$

8891 OE 1-

OZSEZEI -

$976 \tau 8 \varepsilon 1$ - 


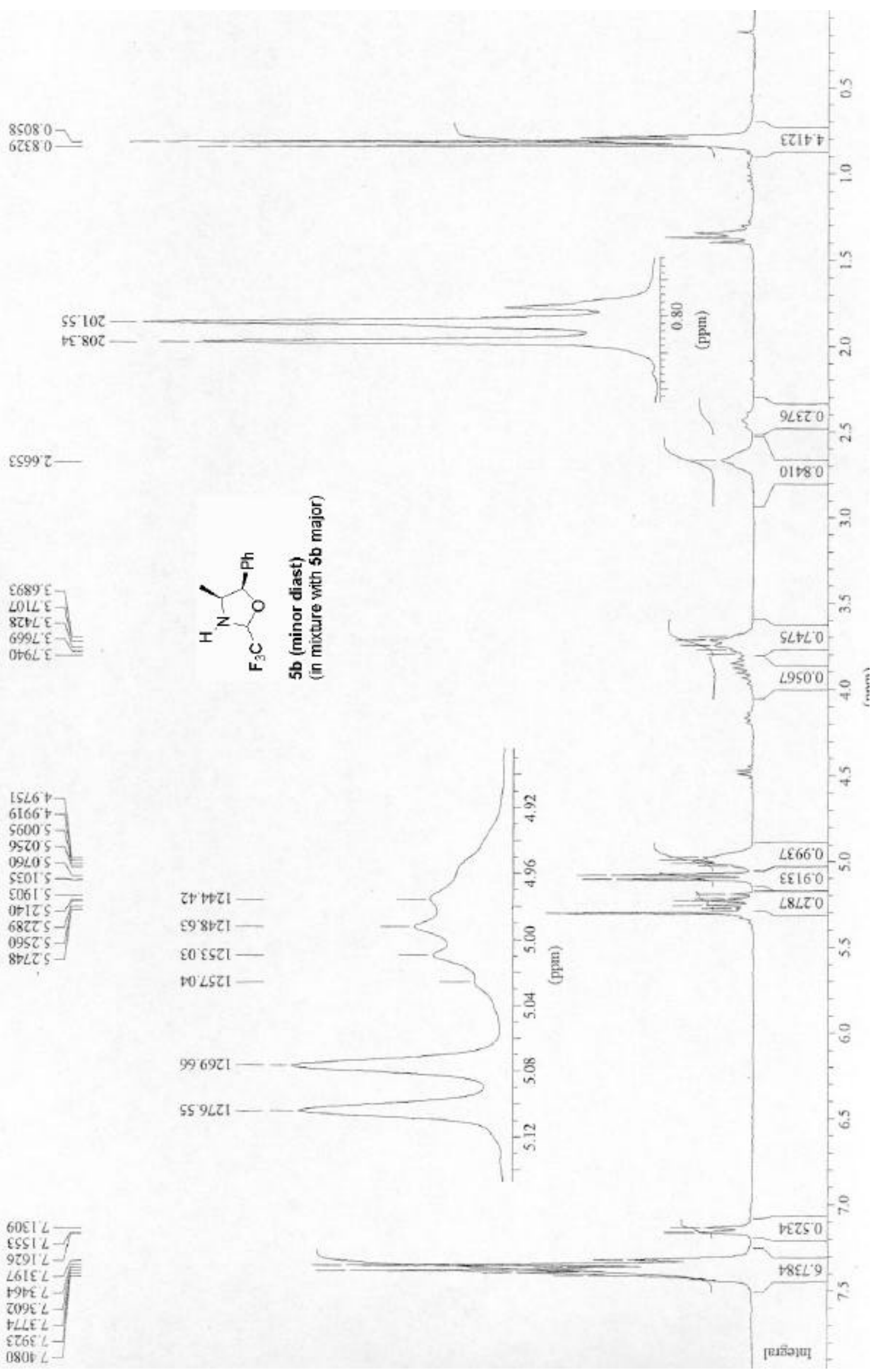


$5 \angle 81^{\circ} 9 L$
$0000 \angle L$

otos:L 느

$90+\angle 28$

$\angle \$ 8298$

tZES 98 .

IGLE LS -

$8526 \angle 8$

9645021

$\angle 800^{\circ} 521$

COI1921 -

$2912221-7]$
$6091821]$

6EOH LEL -

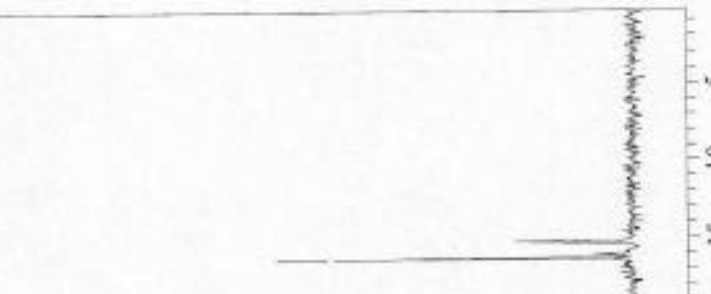

오

$66185 L$

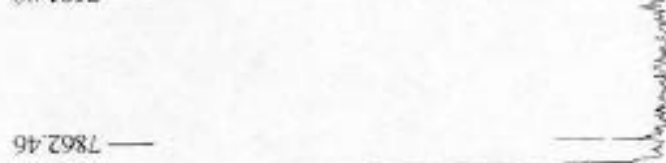

ULIE⿰L

LC Zع08

IL 0908 -

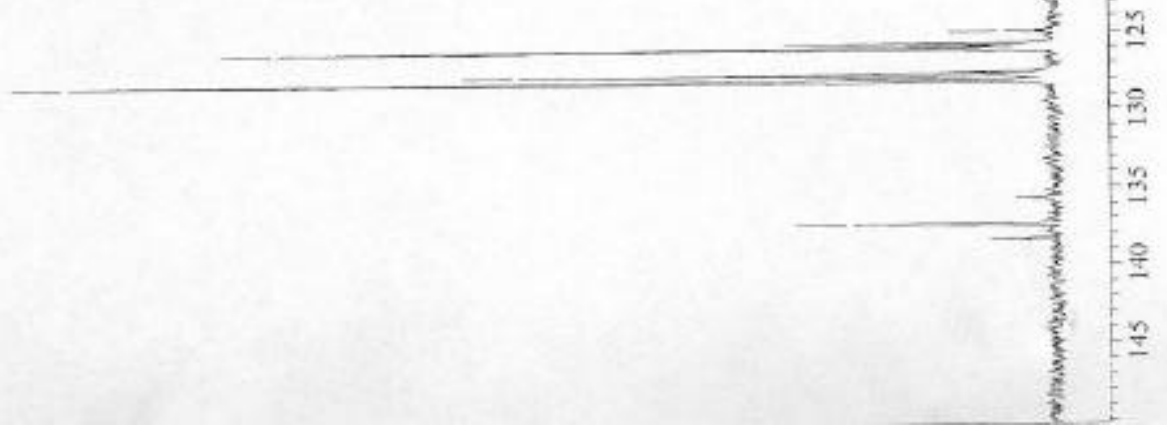


$86590-$

2066 'T -

$8 E O L E$

OIEL'E-

SS8 E $E-1$

$\$ 58 L$ E-
$8218 \mathrm{E}-$

$$
\underbrace{2}_{x_{0}^{2}} \frac{\overline{0}}{\frac{5}{5}}
$$

$5+60^{\circ} \varsigma-$

5001 's

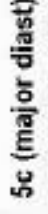

69856

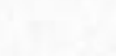

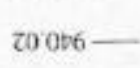

08906

EL80 L -

SLII'L

DILIL

98612

$690 \pi$ L

54 
L012LI

$\$ \$ 160 z-$

SELTDS -

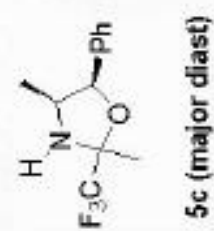

$5885^{-19 L}$

20005 LL 는

521278

6LDDZ6

ptet? \&6 -

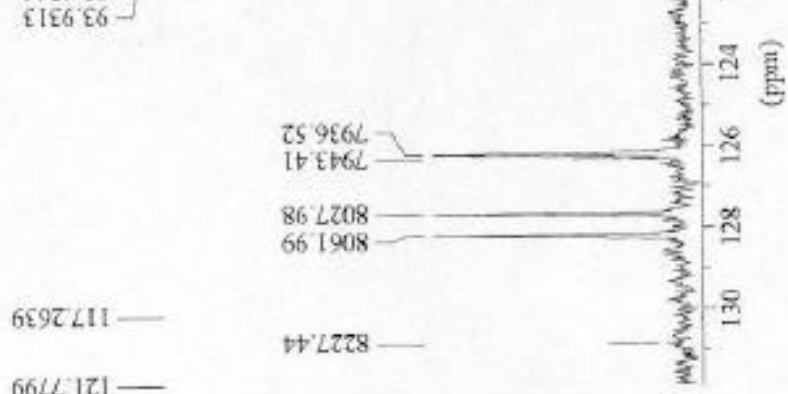

ESt185 -

EEst8s-

859285

E8 2065

$8 \Sigma 6 \leqq 9 L$

$66 L L|Z|=$

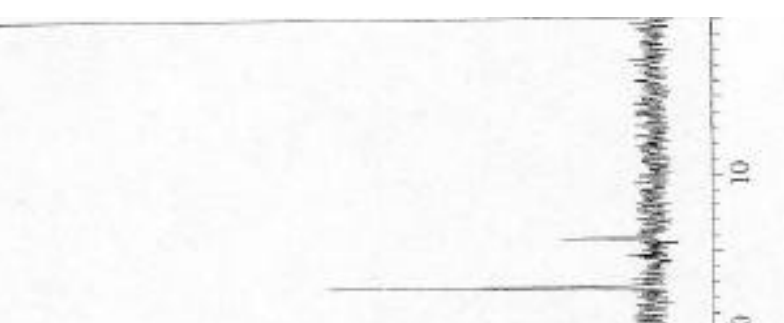

$2981.921-$

s62 921

$60+9 \circ 2 \mathrm{LI}-1$
2181.821

GII80E1

SLSOLEI -

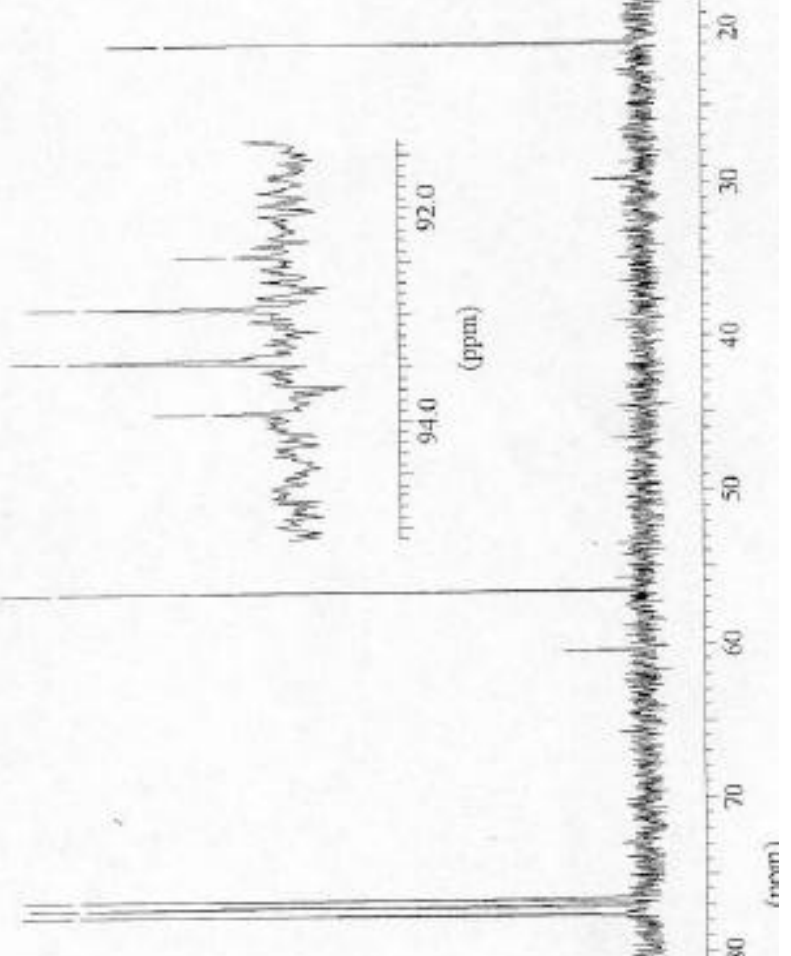

$-8$$$
\text { \& }
$$ 
$2529^{\circ} 0-$

$\operatorname{sis90}=$

$60<9^{\prime} 1$

จระ6!

ostre -

ZZL8'E-

1006 '

9L26:

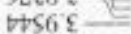

82815
78125

9L196-

55896

ESSL6 -

It 286

Oा 686

$1 \angle 80^{\circ} \mathrm{L}$

DSIIL-E

68116

I861

Z861'

$5022 L L$
$02 Z Z L$

SIDZL -

$8 \mathrm{stz} 2$

$8 E D 22$

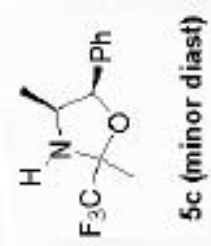

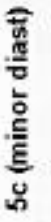

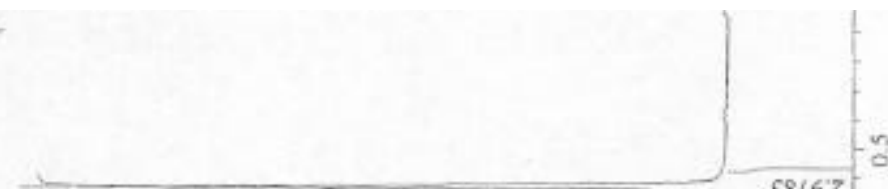

$62280^{-}$

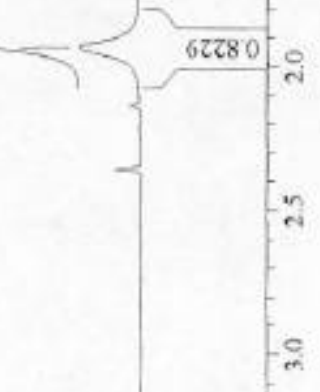

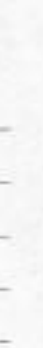

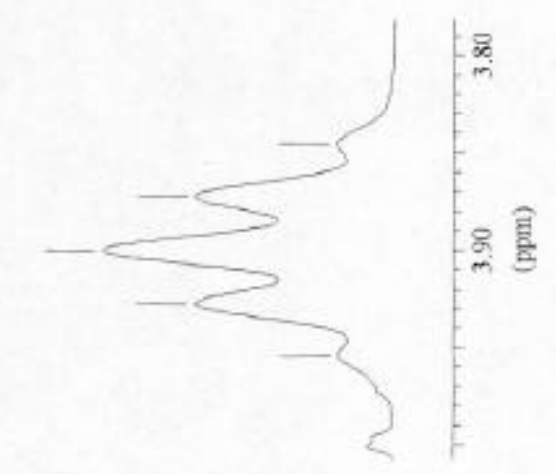

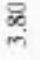


6 เธะ $\angle 1$

$822802-$

$276 \in 25-$

$191+\angle 5+$

$85609 C-$

SIIS LL

8D55 \&8

s2b8:26-

$128586-$

L962 b6-

8621221

$9 E 61^{\circ} 9 \mathrm{ZI}$

$68 \mathrm{IE} L Z \mathrm{ZI}-\mathrm{F}$

LLt9'LZ1

f012821 -

t\&68 IE -

$6946881-$

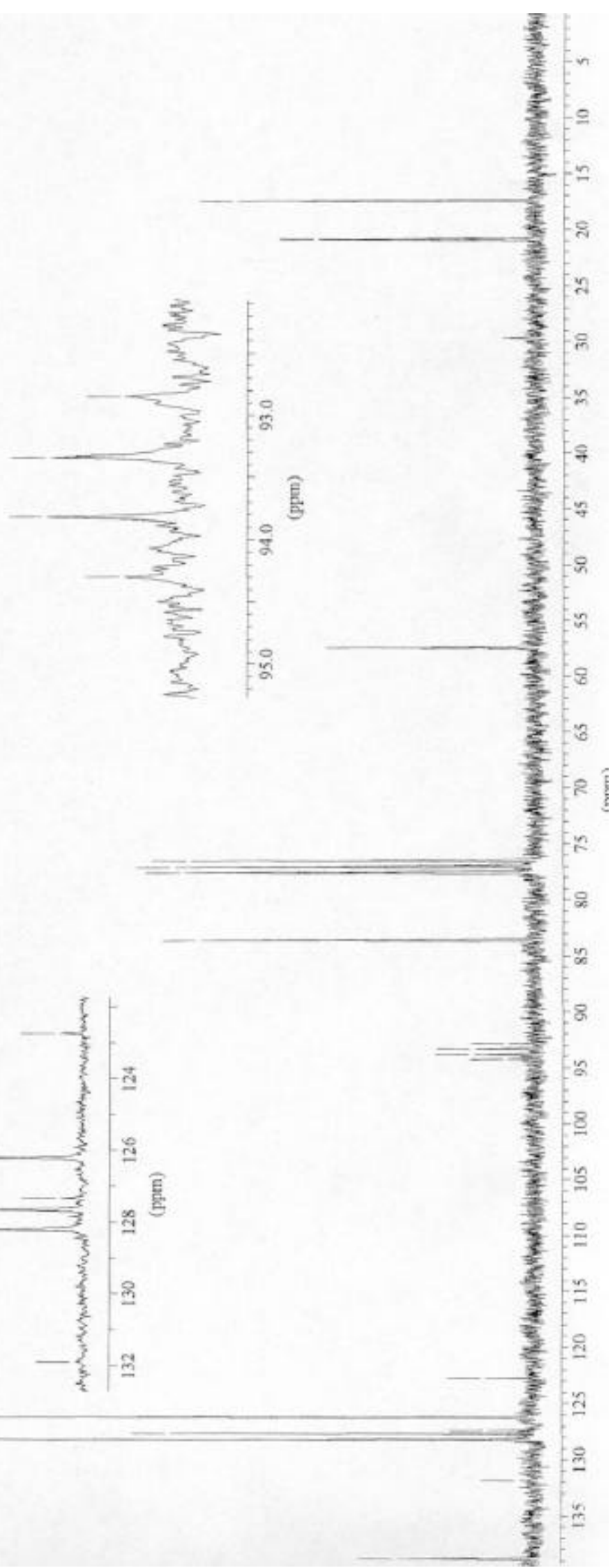


$t 1+2 \mathrm{I}-$

$50 L Z^{\circ} \mathrm{I}=$

22202

ztolz

6LEEZ

$768 \mathrm{t}^{\prime} \mathrm{E}$

$85+C^{\prime} E-$

$80 \angle 9^{\circ} \varepsilon$

SILL'

$8818^{\circ} \mathrm{E}$

$\angle \mathrm{Ht} 8^{\circ} \mathrm{C}$

$0998^{\circ} \mathrm{E}$

$9668^{\circ} \mathcal{E}$

$8 \mathcal{8 1 6} 6^{\circ}$

L866\&

E190't-

$8160^{\circ} t-$

LOZI't -

Lot I't

E98I't

LLOZ

I $2 E 2 t-1$

$6 t \subseteq Z t-1=$

$t \varepsilon \varepsilon t^{\circ}-$

c88t t-

ऽ508't-E

$8 \varepsilon z 8^{\prime} t-$
$9 \varepsilon t 8 t-1=$

$0898^{\circ} \mathrm{t}-$

$8 \angle 88^{\circ} \mathrm{t}-$

$1906 \mathrm{t}=$

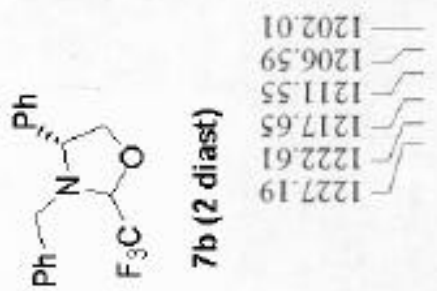

\&68011 -

25\$111-

292211-

8tczol-

$\varepsilon L^{\circ} 0 \varepsilon 0 \mathrm{I}$

86 LEOI

$\varepsilon \mathrm{I} \angle \mathrm{TOI}-$

8 ' $^{2} \mathrm{SOl}-$

$858501-$

0E5901 -

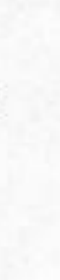

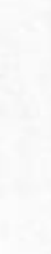




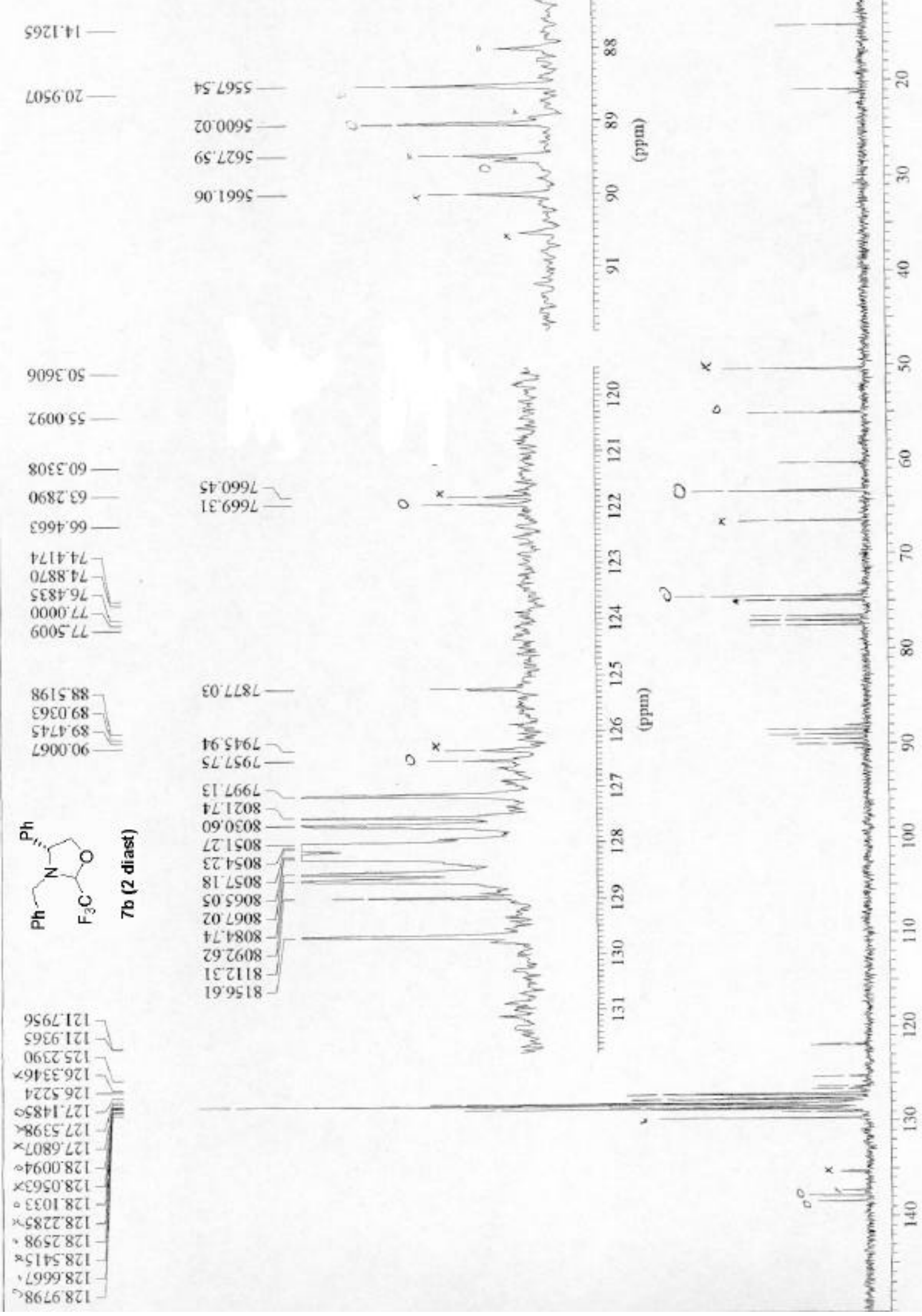




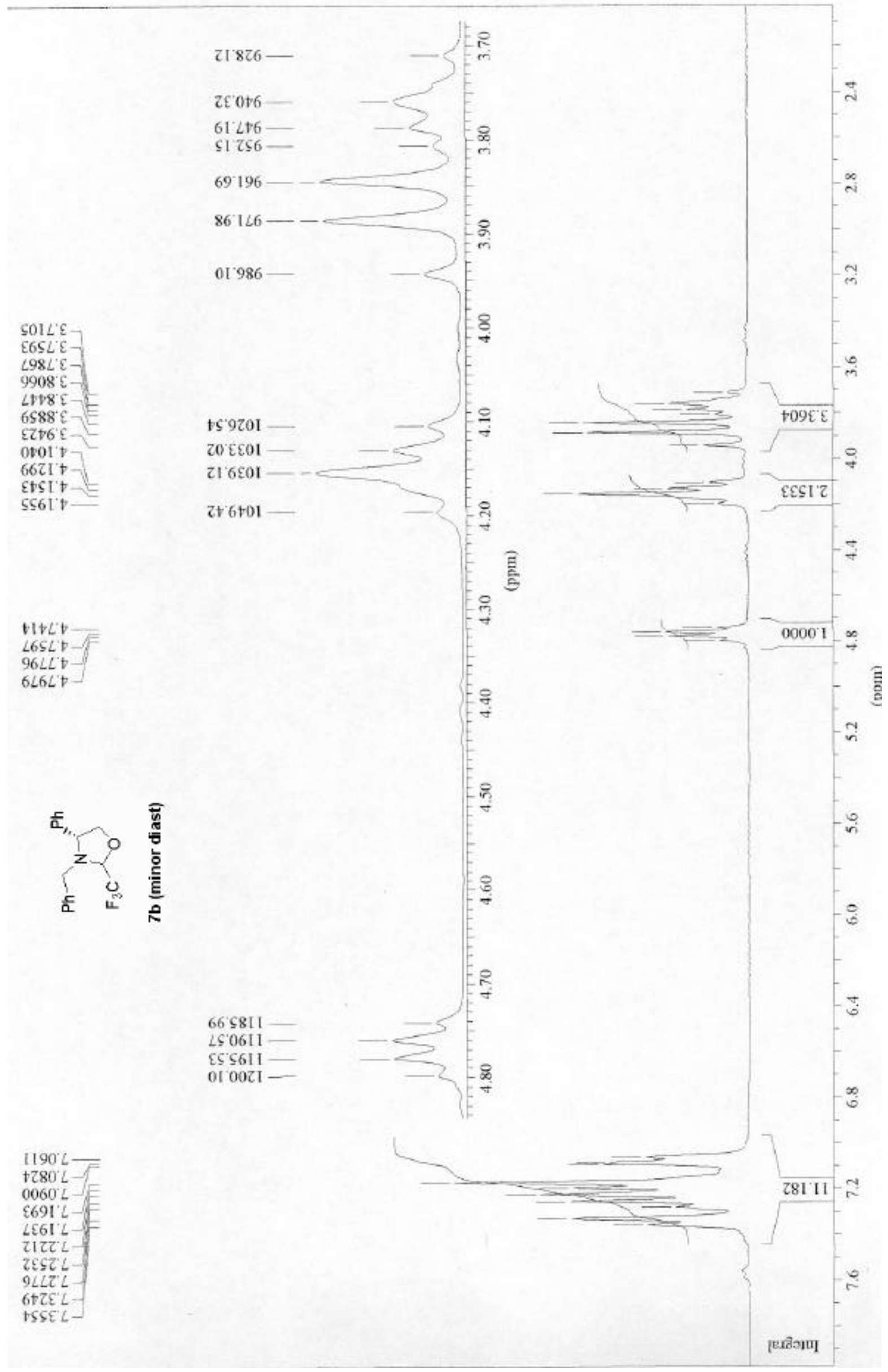


CEGGits

$8996^{\circ} 99$

OVE6 $\mathrm{TL}$

$\$ 88 t 9 L$

$0000^{\circ} \mathrm{LL}$

$6005 \mathrm{LL}$

$\$ 20688$

$S+\angle t^{\prime} 68$

$\angle 900^{\circ} 06-$

ZEZS 06
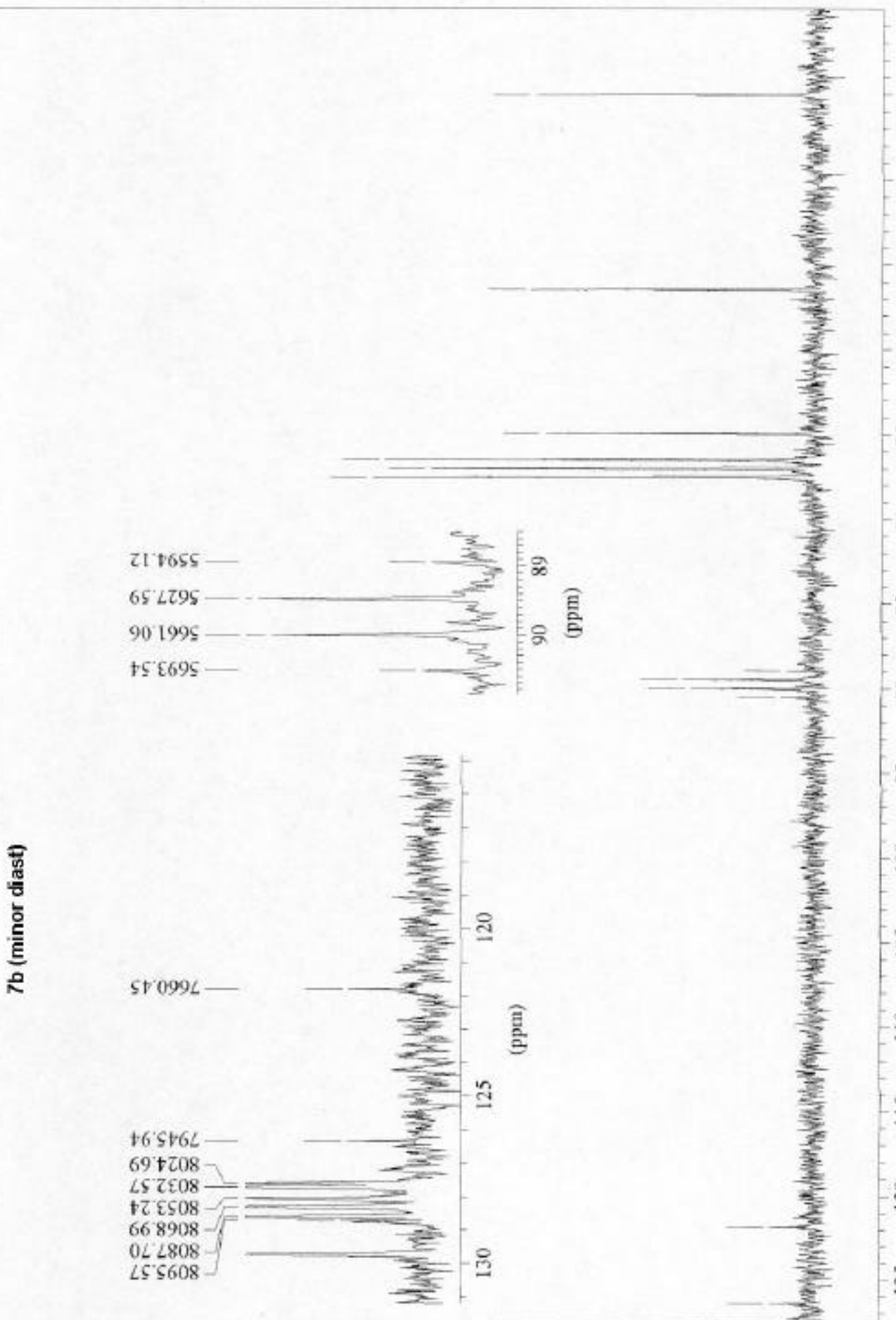

- n

8

$\therefore$

웅

$\approx$

क्ष

$-\infty$

-8

2

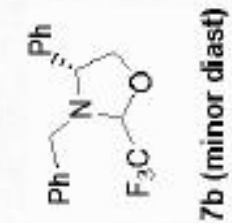

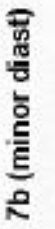

$9 \$ 6 L I Z L$

9tรE 921

$898 S^{\prime} L Z \mathrm{I}$

oz $1 L L Z 1$ -

L0t0 821 -

I $16 z^{\prime} 8 z I-$

$58858 \mathrm{ZI}-$

LEIL $8 Z I$

tsil $62 \mathrm{~L}$ -

IEOESEI-

990ELEI - 


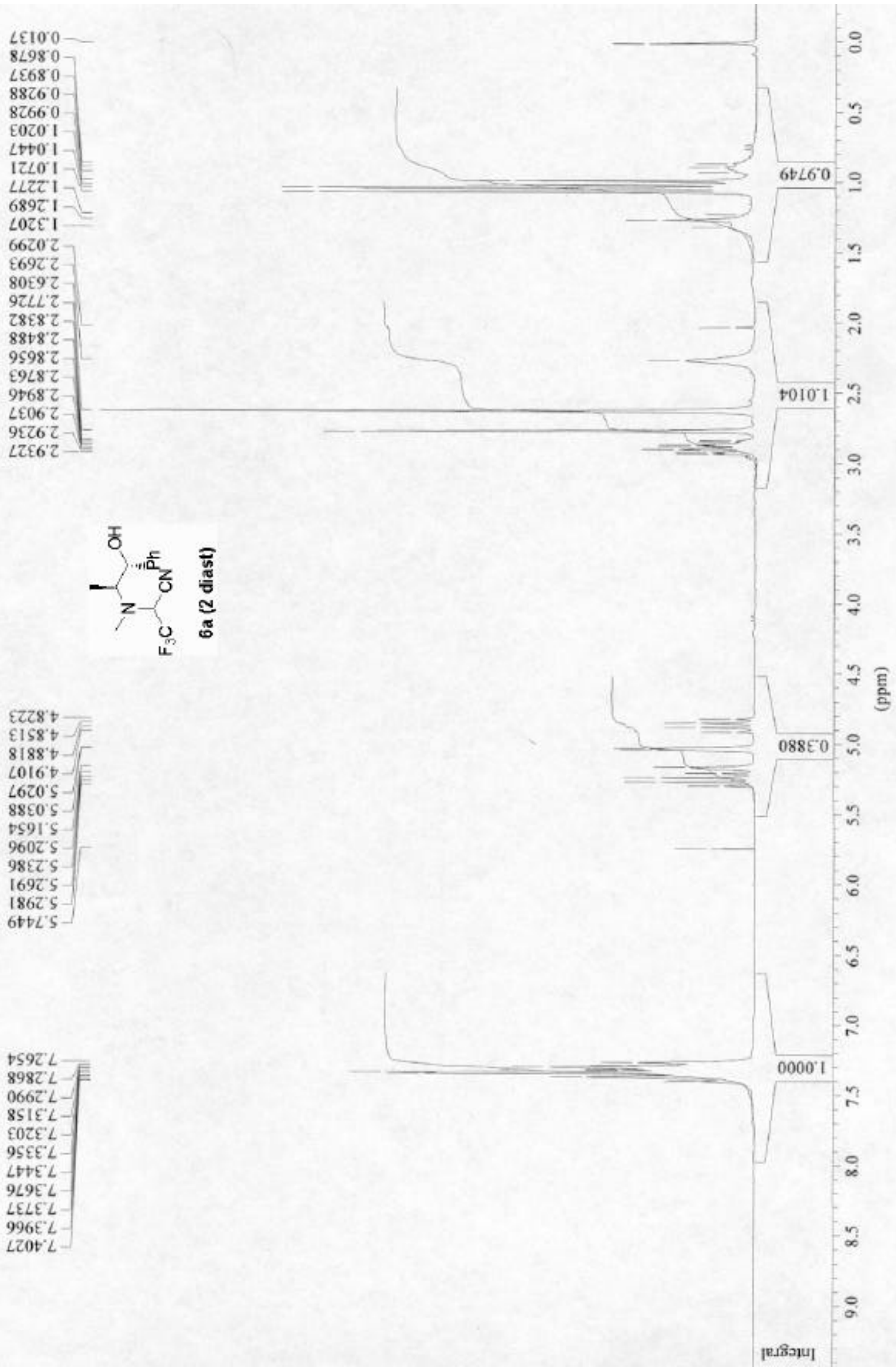



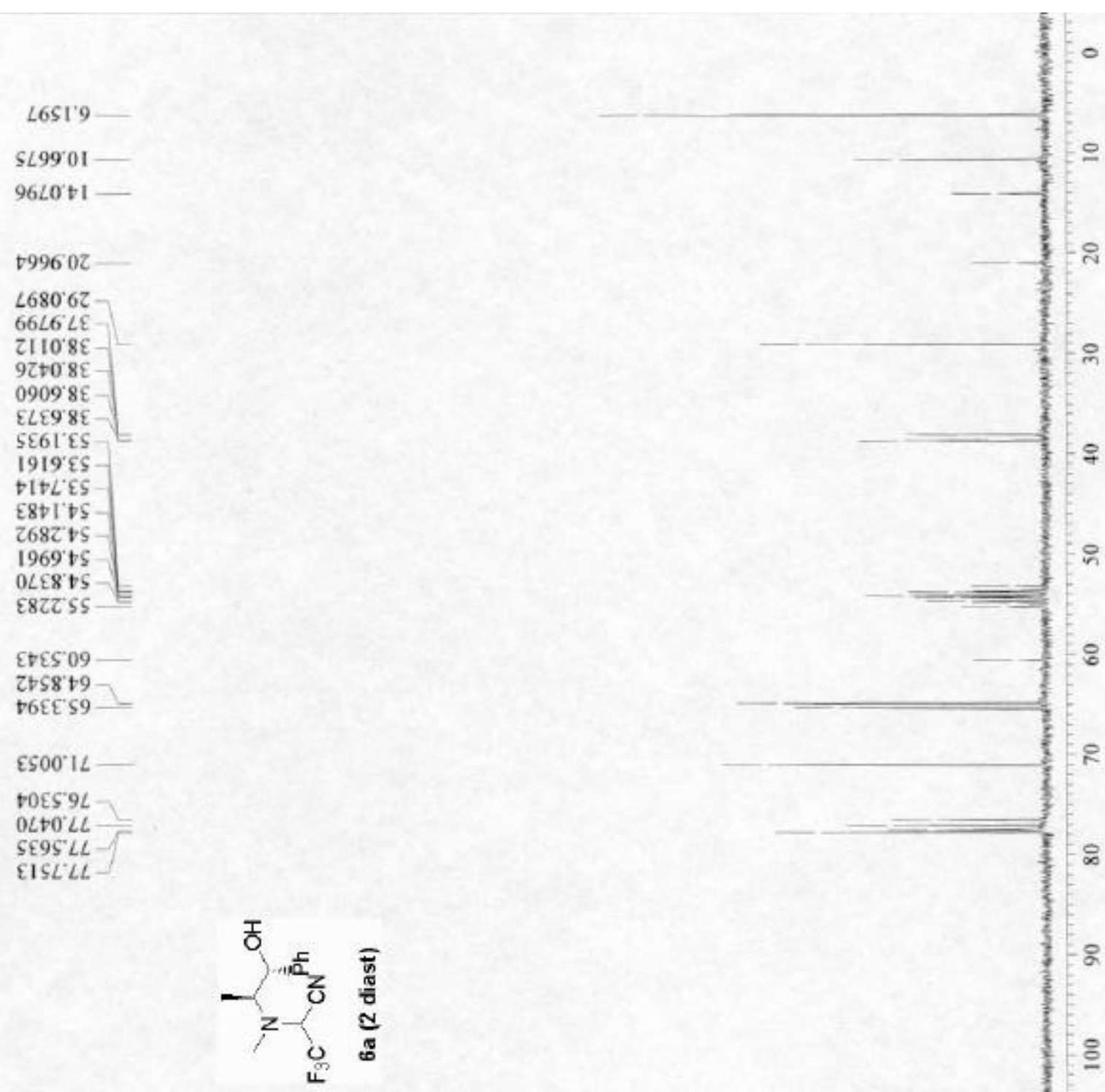

$6+62211$

Stot'zll

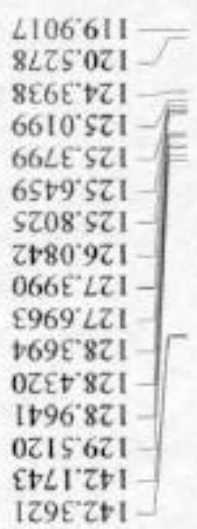



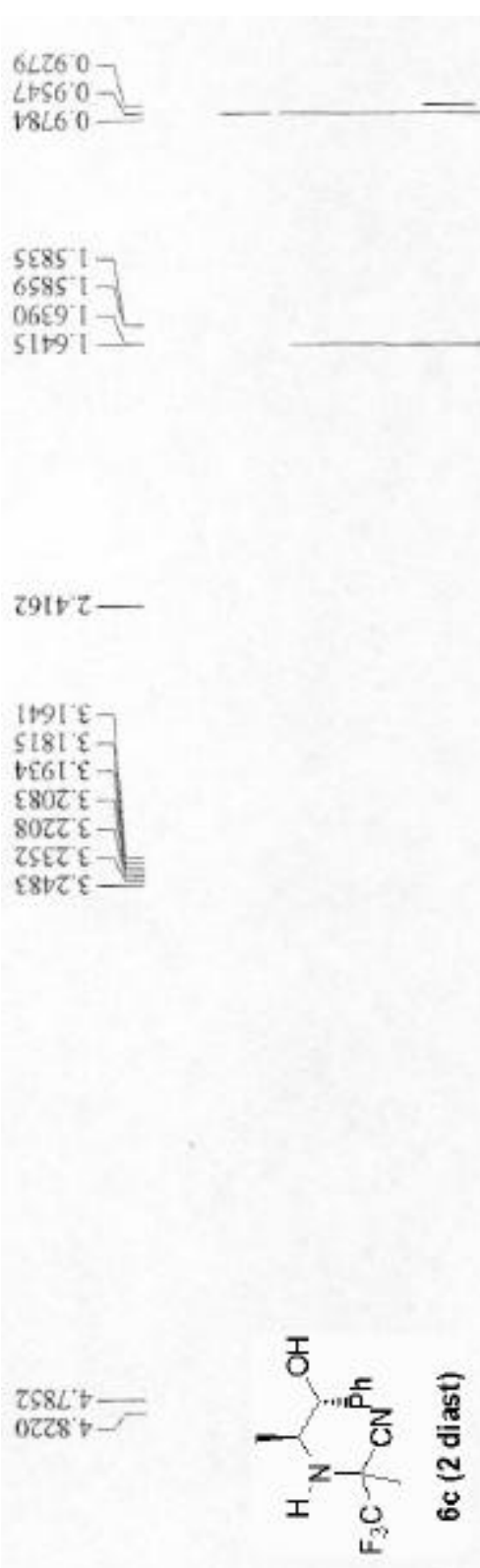

$758 L^{\circ}+-$
$0228^{\circ}+2$

$0181 \%$

$5861 \%$

$8602 \mathrm{~L}-$

$2212^{2} L-$

$\angle 612 L$

CLEZ L

ztez L

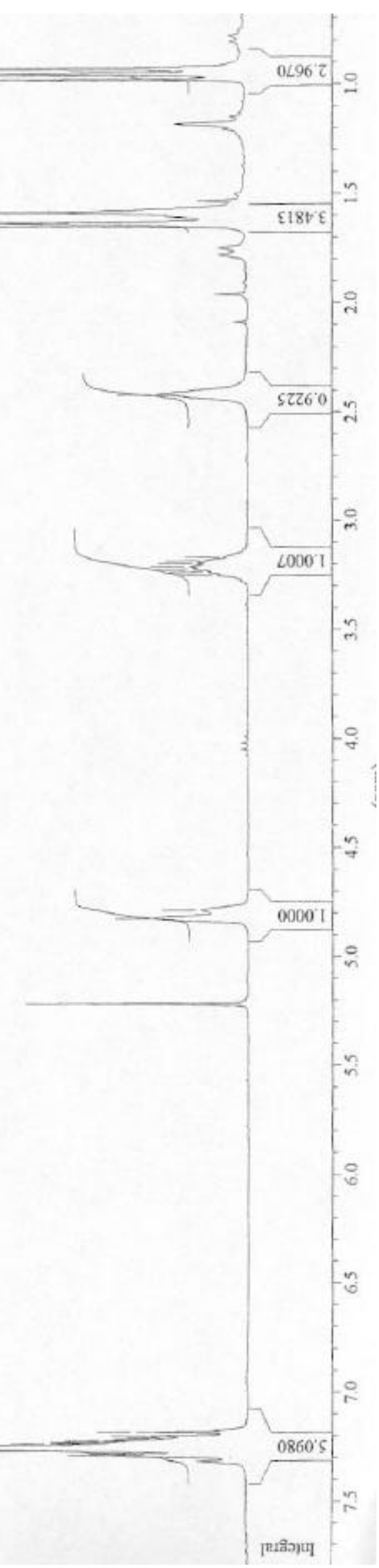


$0191^{-} 51-$

EtE- $51 \div$

เ̇IE 0 LtEZIT-

8EEZSS -

$\$ 586 \$ \$-$

EESOLS

20HS LS -

$1420 ' 85-1$

I 1585

$\$ 96 t S L-$

$8 E 98$ ' L

$096692=$

$00000^{\circ}$

SZIS LL-

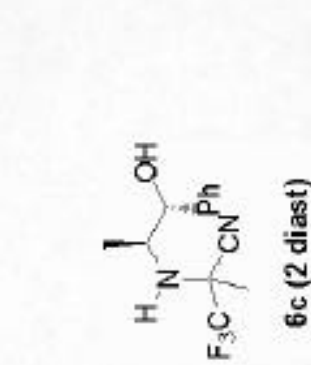

$8895^{\circ} 911-$

EL86.911

9eso' 121

$1185521-$

$65+8521-1-$
2800921

2800921
$z 025[21$

$\varepsilon 8+9 \angle z 1$ -

LtE' $8 Z 1$.

000I OEI -

$\angle 269001-$
Optioni-

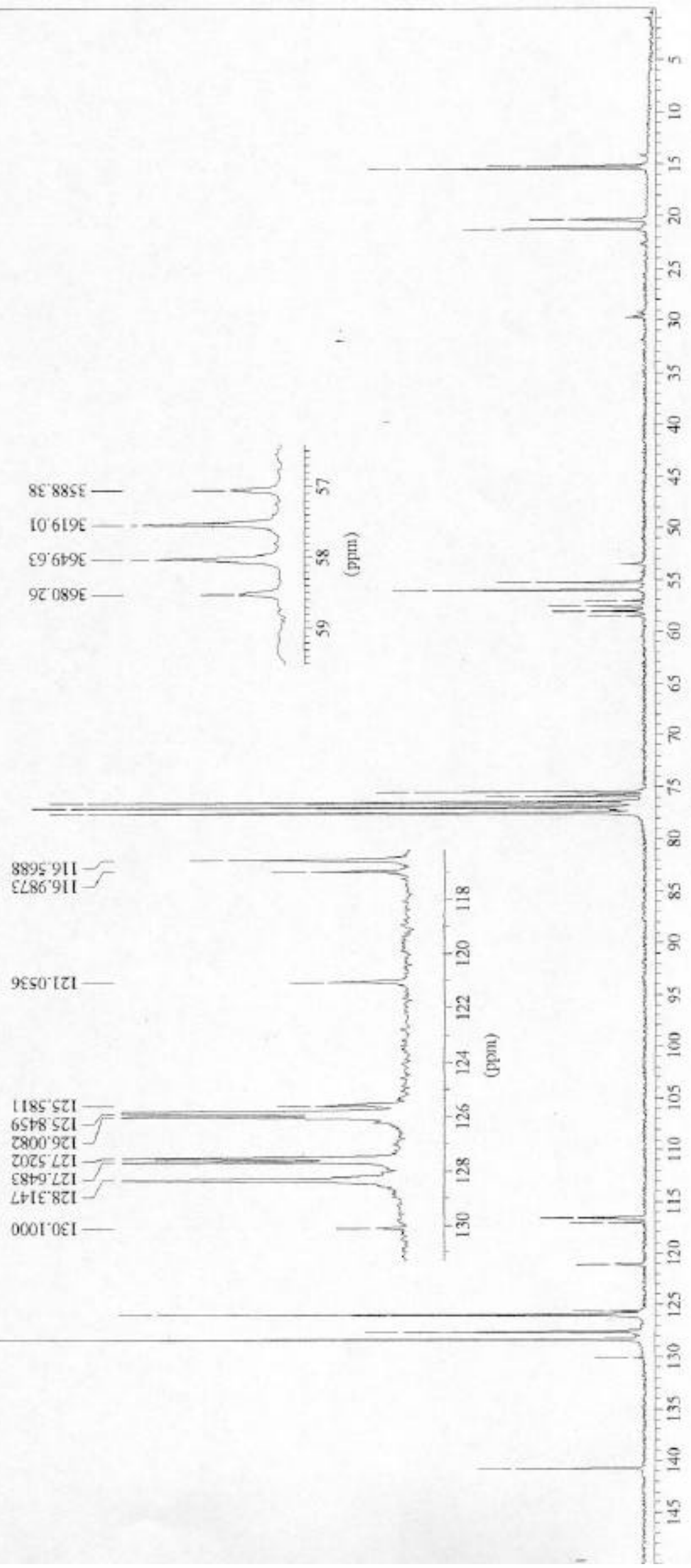




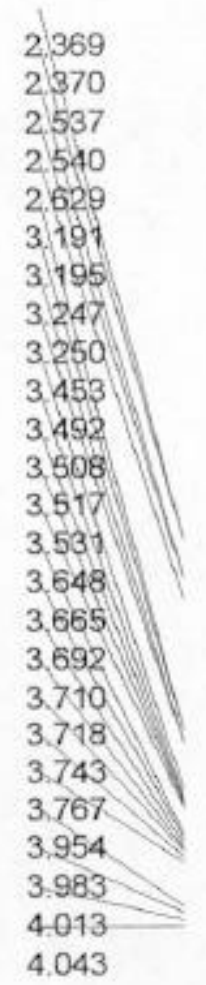

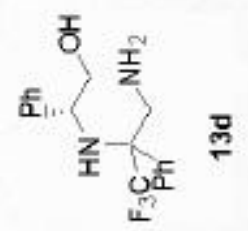


43.547

58.902

65.772

66. 143

66.501

66.881

67.747

76.490

77.000

77.509

든..

119.877

124.475

126.859

127.037

12.976

128.038

128.217

129.071

133.669

135.382

142.786

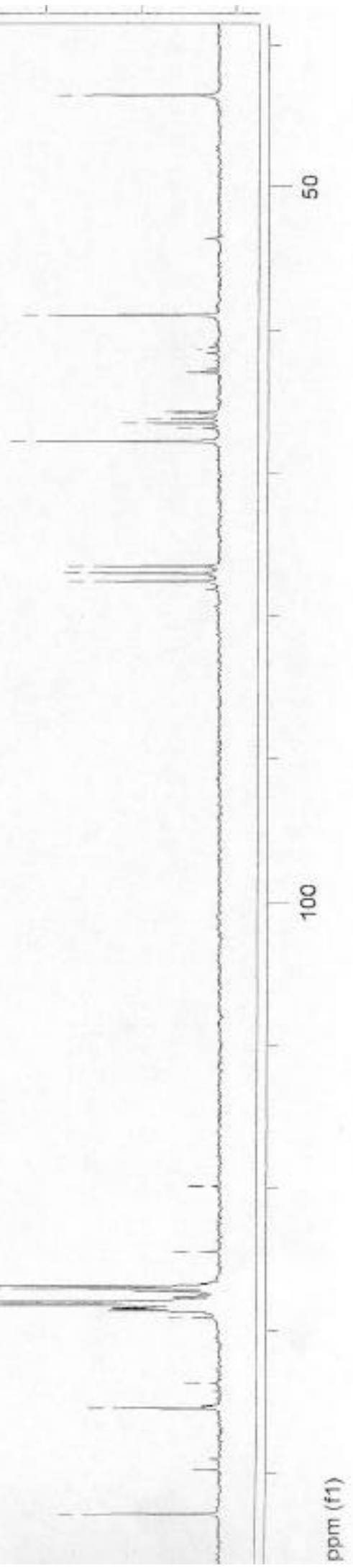


$6+9 L^{\circ} \mathrm{E}$

28825

EEC
$5908^{\circ} \mathrm{E}$

t668\%

$5946^{\circ} \mathrm{s}$

$8456^{\circ}=-$
$6586^{\circ}-$

68

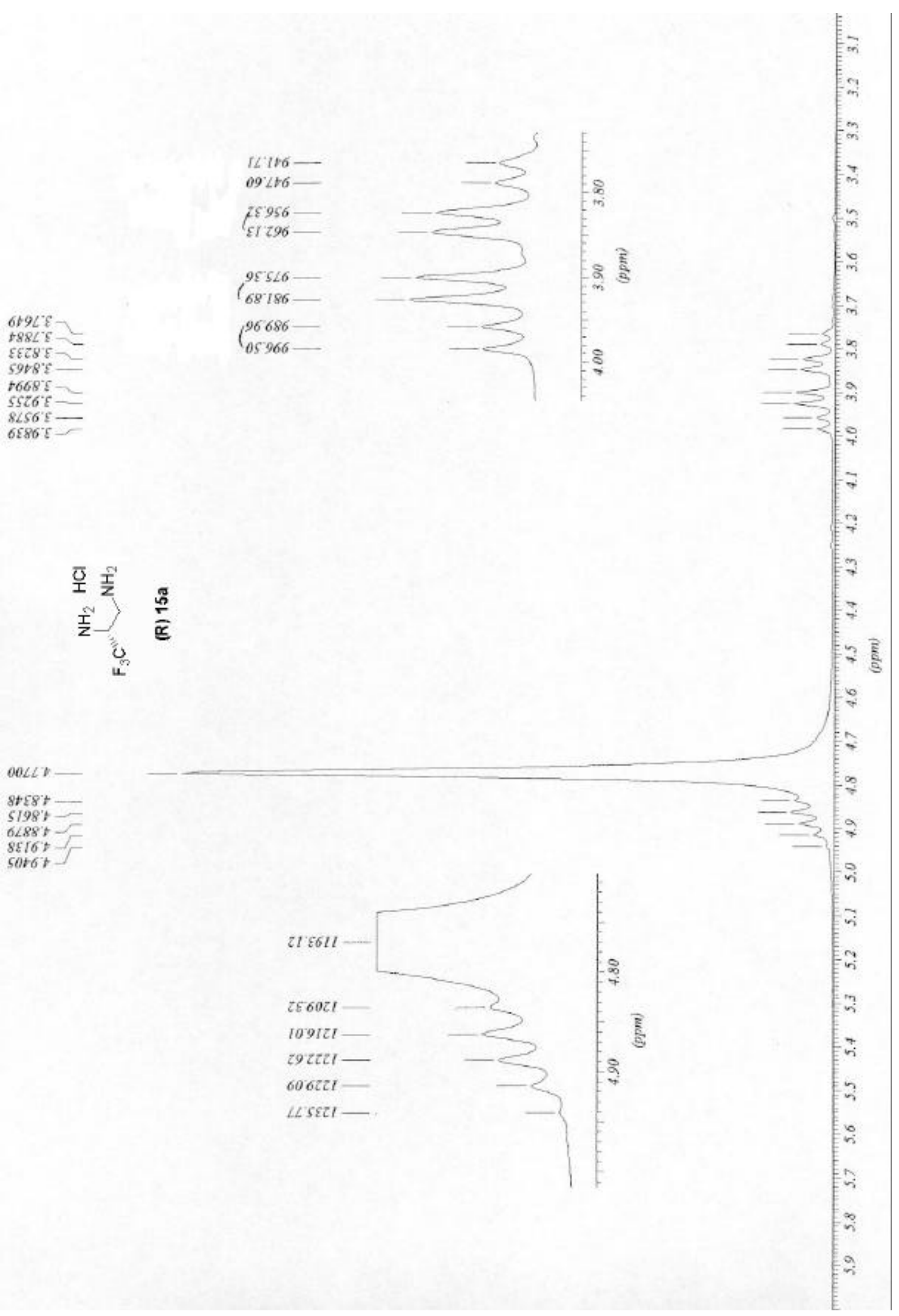


$6 Z \angle \angle 9 E-$
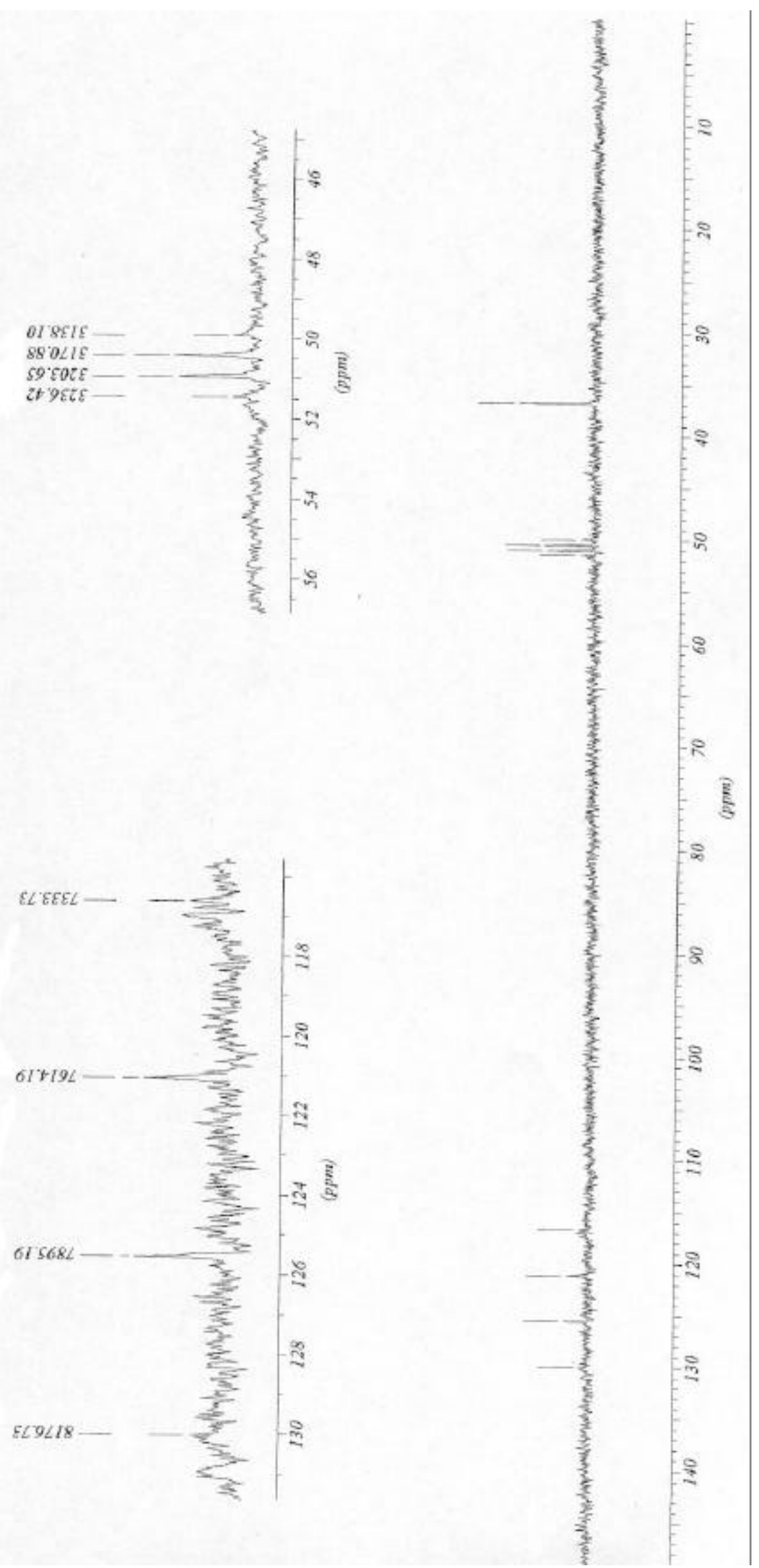

It $6865-$
2SIt $05-$

c956 05 -

$1 / 50.15$

오 폴

等-

总

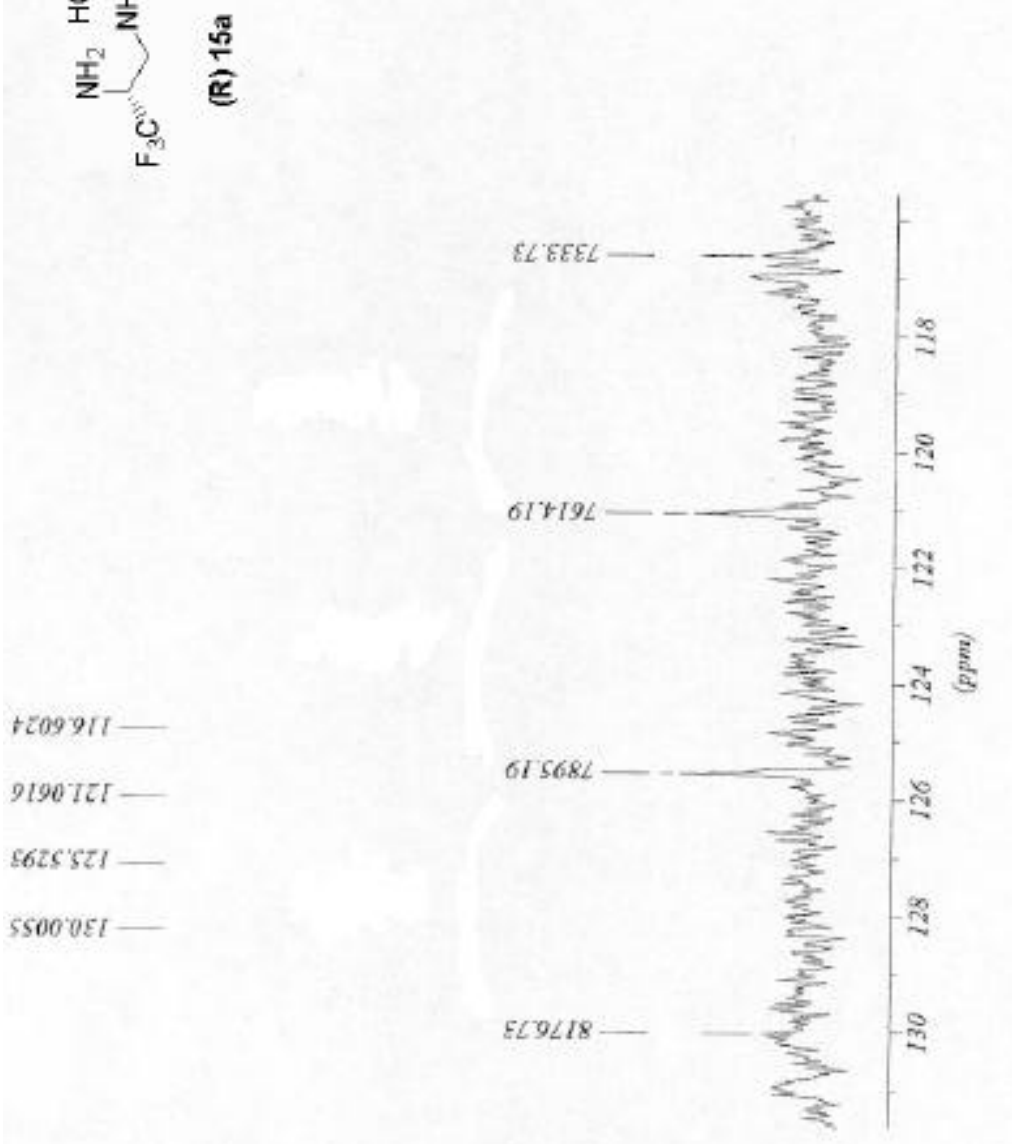



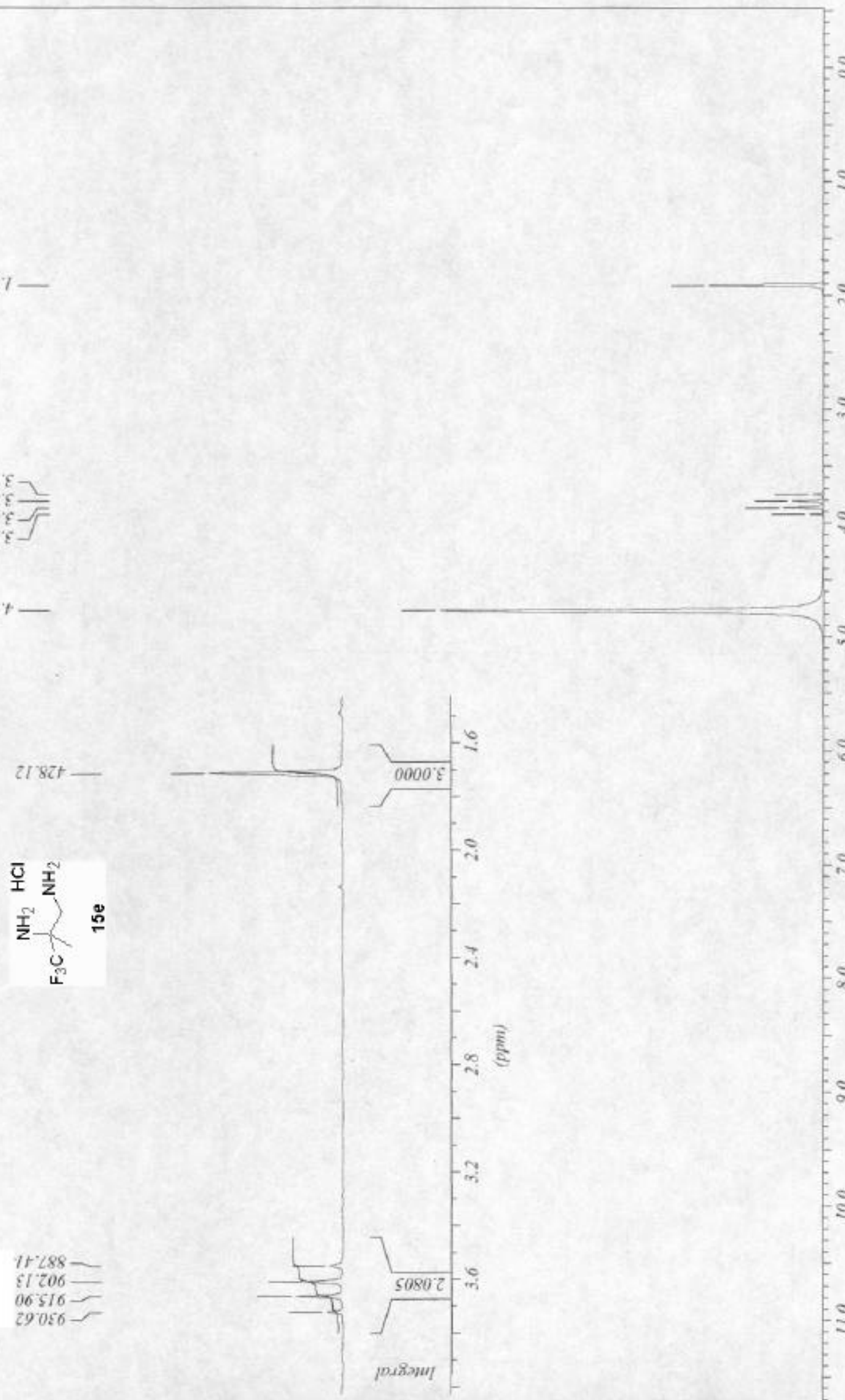


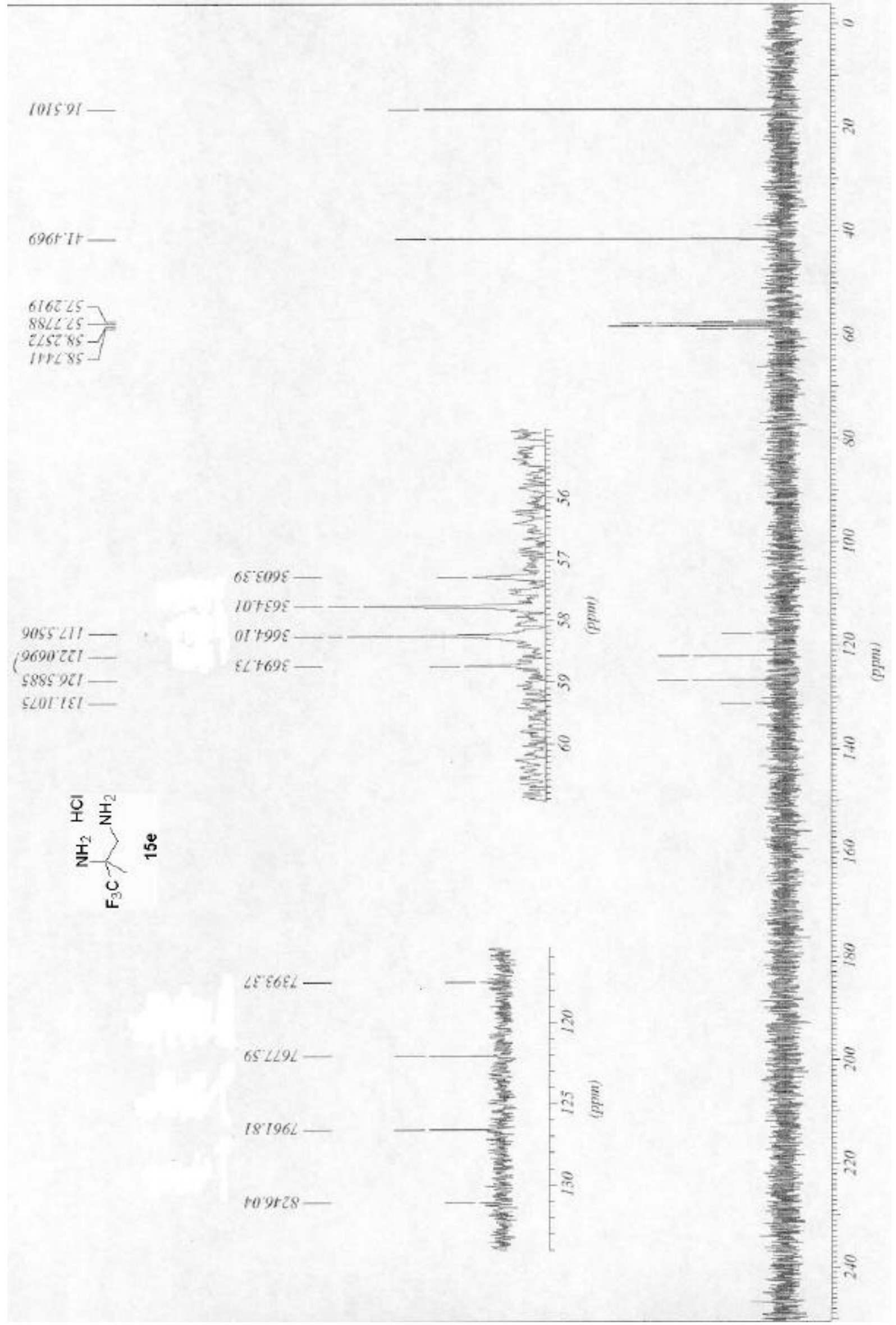

